\title{
Interference effects in medium-induced gluon radiation
}

\author{
J. Casalderrey-Solana ${ }^{a}$ and E. lancu ${ }^{a, b}$ \\ ${ }^{a}$ CERN, Theory Division, \\ CH-1211 Geneva, Switzerland \\ ${ }^{b}$ Institut de Physique Théorique, CEA Saclay, \\ F-91191 Gif-sur-Yvette, France \\ E-mail: jorge.casalderrey@cern.ch, edmond.iancu@cea.fr
}

ABSTRACT: As a step towards understanding the in-medium evolution of a hard jet, we consider the interference pattern for the medium-induced gluon radiation produced by a color singlet quark-antiquark antenna embedded in a QCD medium with size $L$. We focus on the typical kinematics for medium-induced gluon radiation in the BDMPS-Z regime, that is, short formation times $\tau_{f} \ll L$ and relatively large emission angles $\theta \gg \theta_{c} \equiv 2 / \sqrt{\hat{q} L^{3}}$, with $\hat{q}$ the 'jet quenching' parameter. We demonstrate that, for a dipole opening angle $\theta_{q \bar{q}}$ larger than $\theta_{c}$, the interference between the medium-induced gluon emissions by the quark and the antiquark is parametrically suppressed with respect to the corresponding direct emissions. Physically, this is so since the direct emissions can be delocalized anywhere throughout the medium and thus yield contributions proportional to $L$. On the contrary, the interference occurs only between gluons emitted at very early times, within the characteristic time scales for quantum and color coherence between the two emitters, which in this regime are much smaller than $L$. This implies that, for $\theta_{q \bar{q}} \gg \theta_{c}$, the medium-induced radiation by the dipole is simply the sum of the two BDMPS-Z spectra individually produced by the quark and the antiquark, without coherence effects like angular ordering. For $\theta_{q \bar{q}} \ll \theta_{c}$, the medium-induced radiation by the dipole vanishes.

Keywords: Jets, Hadronic Colliders, QCD

ARXIV EPRINT: 1105.1760 


\section{Contents}

1 Introduction $\quad 1$

2 Physical discussion and summary 4

2.1 A primer on BDMPS-Z physics 4

2.2 Qualitative discussion of interference 8

3 General set-up and formalism $\quad 14$

$\begin{array}{ll}3.1 & \text { The amplitude for gluon emission } \\ 3.2 & 14\end{array}$

$\begin{array}{ll}3.2 \text { The 'out-out' terms as a warm up } & 19\end{array}$

4 Medium-induced gluon radiation: direct emission $\quad 23$

5 Medium-induced gluon radiation: interference terms 30

$\begin{array}{lll}6 & \text { Discussion and outlook } & 37\end{array}$

$\begin{array}{ll}\text { A Momentum space analysis of the gluon spectrum } & 41\end{array}$

A.1 The gluon spectrum at the time of formation 42

A.2 The final gluon spectrum 44

A.3 Direct emission: the BDMPS-Z spectrum 45

A.4 The interference terms for relatively large dipoles: $\theta_{f} \lesssim \theta_{q \bar{q}} \lesssim \theta_{s} \quad 45$

A.5 The interference terms for relatively small dipoles: $\theta_{c} \ll \theta_{q \bar{q}} \ll \theta_{f} \quad 46$

\section{Introduction}

The phenomenon of jet quenching globally denotes the modifications in the properties of a jet which occur when the jet propagates through the dense QCD matter created in the intermediate stages of a ultrarelativistic heavy ion collision. One of the most striking effects of this kind is the large di-jet asymmetry observed in $\mathrm{Pb}+\mathrm{Pb}$ collisions at the LHC, as reported by the ATLAS [1] and CMS [2] collaborations (see also [3] for related results at RHIC). These data imply that, as a consequence of the interactions between the jet and the medium, the jet energy is transported to larger angles and redistributed into softer fragments as compared to the $\mathrm{p}+\mathrm{p}$ baseline. Understanding this phenomenon of strong jet broadening and also the strong suppression of particle production at high $p_{T}$ in nucleusnucleus collisions as compared to $\mathrm{p}+\mathrm{p}$, as observed at RHIC [4-7] and the LHC [8], is essential for using jet probes as a diagnosis tool of hot and dense QCD matter.

From a microscopic point of view, the dominant mechanism for jet quenching at weak coupling and high energy is radiative energy loss associated with medium-induced gluon radiation [9-17] (see also the review papers [18, 19] for more references). If the medium 
is sufficiently dense, both the parton that initiates the jet and its descendants undergo multiple scattering leading to additional radiation which is described by the BDMPS-Z (from Baier, Dokshitzer, Mueller, Peigné, Schiff, and Zakharov) formalism. While this mechanism for jet quenching has been quite successful in describing the suppression of single particle spectra observed at RHIC (see, for example [20, 21]), it has been realized for long that the respective data refer to inclusive measurements which are quite limited in constraining the underlaying dynamics. By contrast, the differential jet measurements that are performed at the LHC provide more detailed informations, in particular, on the spectrum of the medium-induced radiation which could help us to better pinpoint the physical mechanisms at work.

At this point, one should stress that the BDMPS-Z mechanism predicts that gluons are emitted at relatively large angles - the softer the gluon, the larger its emission angle - and thus it has the potential to explain the di-jet asymmetry measured at the LHC (see the recent publications [22-25] for related studies). However, from the experience with jet evolution in the vacuum, one knows that large angle radiation can be prohibited by coherence effects leading to angular ordering: within the partonic cascade produced via jet fragmentation in the vacuum, the emission angles are bound to decrease from one emission to the next one. So far very little is known about the corresponding property for the medium-induced gluon radiation. The only analyses in that sense so far $[26,27]$ are either restricted to the single-scattering approximation [26] or concerned with a different mechanism for medium-induced radiation [27], which applies to relatively soft and collinear emissions which are less effective in broadening the transverse energy distribution of a jet.

It is therefore crucial to clarify whether interference effects can frustrate mediuminduced radiation at large angles, in the interesting regime where the medium is relatively opaque and the multiple scattering is important. This is the main objective in this paper. To that aim, we shall study the interference between the medium-induced gluon emissions by two sources immersed into the medium: a quark $(q)$ and an antiquark $(\bar{q})$. More precisely, we shall address the problem of the in-medium 'dipole antenna pattern', that is, the radiation produced by a $q \bar{q}$ pair in a color singlet state (a 'color dipole') where the two particles separate from each other at constant velocities which make a relative angle $\theta_{q \bar{q}}-$ the dipole opening angle. This 'dipole antenna' is a familiar set-up for studies of interference and angular ordering for radiation in the vacuum [28, 29] and has been generalized in refs. $[26,27]$ to corresponding studies in a medium. As usual in the related literature, we shall work in the 'multiple soft scattering approximation' which assumes that successive scattering centers are independent from each other. Formally, the results of ref. [26] can be recovered from this formalism as the lowest order term in the 'opacity expansion', that is, the perturbative expansion of the medium effects. But this is only formal, since the effects of multiple scattering are non-perturbative and the final results cannot be expanded out anymore $^{1}$ (see e.g. the discussion in [31]). In that sense, we expect our conclusions to differ from those in ref. [26] at qualitative level, and not only quantitatively.

\footnotetext{
${ }^{1}$ This is similar to the failure of the twist expansion for high-energy scattering in the vicinity of the unitarity limit, or in the gluon saturation region [30].
} 
The main conclusion which emerges from our analysis is that the interference effects for medium-induced gluon radiation are parametrically small and hence irrelevant for all values of the dipole angle $\theta_{q \bar{q}}$ except for very small values $\theta_{q \bar{q}} \lesssim \theta_{c}$, where direct emissions and interference terms become comparable with each other, and even cancel each other when $\theta_{q \bar{q}} \ll \theta_{c}$.

In order to explain this conclusion and in particular the special angle $\theta_{c}$, we need to first recall some basic features of the BDMPDS-Z mechanism (see section 2 for a physical discussion). The corresponding phase-space is characterized by two limiting values, a maximal frequency $\omega_{c}$ and a minimum angle $\theta_{c}$, which are expressed in terms of the medium properties as $\omega_{c}=\hat{q} L^{2} / 2$ and $\theta_{c}=2 / \sqrt{\hat{q} L^{3}}$. ( $\hat{q}$ is the jet quenching parameter and $L$ is the longitudinal extent of the slice of the medium which is crossed by the dipole.) The energy loss by the leading particle is dominated by the emission of relative hard gluons with $\omega \simeq \omega_{c}$, but such gluons make a small angle $\theta \simeq \theta_{c}$ with respect to their source and thus are not effective in broadening the jet energy distribution in the transverse plane. Rather, the dominant transverse broadening comes from softer gluons with $\omega \ll \omega_{c}$, which are emitted at relatively large angles $\theta \gtrsim \theta_{f}(\omega) \gg \theta_{c}$. Here, $\theta_{f}(\omega)$ is the minimal emission angle for a gluon with frequency $\omega$ (the 'formation angle') and increases when decreasing $\omega$ below $\omega_{c}$.

An important property of these soft gluons, which is favorable too for the physics of jet broadening, is the fact that they are promptly emitted: the corresponding formation time is much smaller than the medium length $L$. Hence, such gluons can be emitted at any point inside the medium. Accordingly, the longitudinal phase-space for direct emissions by the quark or the antiquark is proportional to $L$. By contrast, the interference between the two partonic sources occurs only for the gluons emitted at sufficiently early times $t<\tau_{\min }$, when the quark and the antiquark are still close enough to each other to ensure color and quantum coherence. The precise mechanism which determines $\tau_{\min }$ depends upon the value of the dipole angle $\theta_{q \bar{q}}$ : (i) when $\theta_{c} \ll \theta_{q \bar{q}} \ll \theta_{f}(\omega)$, the interference is limited by the color decoherence of the $q \bar{q}$ pair (the two sources suffer different color precessions in the medium); (ii) when $\theta_{q \bar{q}} \gtrsim \theta_{f}(\omega), \tau_{\text {min }}$ is rather determined by the condition of quantum coherence (the radiated gluon must overlap with both sources). But in both cases, i.e. so long as $\theta_{q \bar{q}} \gg \theta_{c}$, this upper limit $\tau_{\text {min }}$ is much smaller than $L$, meaning that the phasespace for interference, which is proportional to $\tau_{\text {min }}$, is parametrically suppressed relative to that for direct emissions. Then the interference effects are negligible. On the other hand, when $\theta_{q \bar{q}} \lesssim \theta_{q}, \tau_{\min }$ becomes as large as $L$, so the interference is not suppressed anymore. But then the total medium-induced radiation vanishes, since a gluon emitted at an angle $\theta \gtrsim \theta_{f}(\omega) \gg \theta_{c}$ 'sees' the total color charge of the $q \bar{q}$ pair, which is zero.

To summarize, the medium-induced radiation by the dipole is non-zero only when $\theta_{q \bar{q}} \gg \theta_{c}$ and in that case it is simply the sum of the two BDMPS-Z spectra separately produced by the two emitters, without any coherence effect like angular ordering. In order to substantiate this conclusion and the above physical picture, we shall explicitly estimate the contribution of the interference effects to the spectrum of the medium-induced radiation by the dipole and compare the result with the corresponding contribution due to direct emissions. Our main results in that sense, namely eq. (5.14) for the contribution of the 
interference terms and eq. (4.21) for that of the direct emissions, are confirmed by two different calculations (one exposed in the main text, the other one in the appendix), which involve approximation schemes with different degrees of rigor, but which agree with each other to parametric accuracy.

Our paper is organized as follows: in section 2 we present a qualitative discussion of the medium-induced radiation, including the BDMPS-Z mechanism (for completeness and pedagogy), but focusing on our original results on interferences. Our purpose there is to motivate our conclusions via physical considerations, which hopefully will provide the guidelines for the subsequent, more formal, developments. In section 3 , we give a streamlined presentation of the BDMPS-Z formalism adapted to the problem at hand and also make contact with the analysis in ref. [27]. Sections 4 and 5 are the main sections of this paper. They present detailed calculations of the medium-induced contributions to direct emissions (section 4) and to the interference terms (section 5). To keep the presentation as fluent as possible, in these sections we resort on analytic approximations which are correct at parametric level. (More refined versions of these calculations are deferred to appendix A.) This allows us to provide an explicit expression for the BDMPS-Z spectrum (consistent with the respective results in the literature) and to deduce an equally explicit result for the interference contribution to the spectrum. Finally, in section 6 we discuss the implications of our results for the in-medium evolution of a hard jet and we mention some open problems.

Note added. When this work was already finished, a preprint appeared, ref. [32], in which the general formula for the interference contribution to the medium-induced radiation by the dipole (our eq. (5.2)) was also derived. However, the physical consequences of this formula were not explicitly worked out. In particular ref. [32] did not identify the physical mechanism responsible for the suppression of the in-medium interference terms, which is the reduction in the corresponding longitudinal phase-space. Also the conclusions drawn there by inspection of the general formula turned out to be incorrect in some cases.

\section{Physical discussion and summary}

We start our presentation with a section which summarizes, at a qualitative level, the physical picture and the main conclusions that we shall eventually reach through our analysis. This discussion will motivate the subsequent, more formal, manipulations and hopefully provide the guidelines for the approximations to come. It will also allow us to introduce the various time scales which control the dynamics and which for the benefit of the reader are summarized in table 1.

\subsection{A primer on BDMPS-Z physics}

The propagation of a high energy parton through a dense QCD medium leads to energy loss and transverse momentum broadening via medium-induced radiation, that is, the emission of gluons stimulated by the interactions between the quark, or the radiated gluon, and the medium. The radiation process requires a characteristic formation time which can be 


\begin{tabular}{|cccl|}
\hline Parameter & Definition & Parametric estimate & Physical meaning \\
\hline$\tau_{q}$ & $\frac{2 \omega}{k_{\perp}^{2}}$ & $\tau_{f}\left(\frac{\theta_{f}}{\theta_{q}}\right)^{2}$ & vacuum formation time \\
\hline$\tau_{f}$ & $\sqrt{\frac{2 \omega}{\hat{q}}}$ & $\sqrt{\frac{\omega}{\omega_{c}}} L$ & in-medium formation time \\
$\theta_{f}$ & $\left(\frac{2 \hat{q}}{\omega^{3}}\right)^{1 / 4}$ & $\theta_{c}\left(\frac{\omega_{c}}{\omega}\right)^{3 / 4}$ & formation angle \\
$\theta_{s}$ & $\frac{\sqrt{\hat{q} L}}{\omega}$ & $\theta_{c} \frac{\omega_{c}}{\omega}$ & saturation angle \\
\hline$\tau_{\text {int }}$ & $\frac{2}{\omega \theta_{q \bar{q}}^{2}}$ & $\tau_{f}\left(\frac{\theta_{f}}{\theta_{q \bar{q}}}\right)^{2}$ & interference time \\
$\tau_{\lambda}$ & $\frac{1}{\theta_{q \bar{q}}(\omega \hat{q})^{1 / 4}}$ & $\tau_{f} \frac{\theta_{f}}{\theta_{q} \bar{q}}$ & transverse resolution time \\
$\tau_{\text {coh }}$ & $\frac{2}{\left(\hat{q} \theta_{q \bar{q}}^{2}\right)^{1 / 3}}$ & $\tau_{f}\left(\frac{\theta_{f}}{\theta_{q}}\right)^{2 / 3}$ & color decoherence time \\
\hline
\end{tabular}

Table 1. Scales relevant for medium-induced gluon radiation. The dimensionless ratios are related to the BDMPS medium parameters $\omega_{c}=\hat{q} L^{2} / 2$ and $\theta_{c}^{2}=4 / \hat{q} L^{3}$.

understood as the time for the (virtual) gluon to separate enough from its parent quark for the quantum coherence between the two quanta to be lost. This formation time $\tau_{q}$ can be estimated from the condition that the transverse separation $b_{\perp}=\tau_{q} v_{\perp}$ between the quark and the gluon at the formation time be of the order of the gluon transverse wavelength $\lambda_{\perp}=1 / k_{\perp}$. Here 'transverse' refers to the direction orthogonal to the trajectory of the quark, $k_{\perp}$ is the gluon transverse momentum and $v_{\perp}=k_{\perp} / \omega$ is its transverse velocity. We have also introduced the gluon energy $\omega$, assumed to be large compared to $k_{\perp}$. Accordingly, the gluon emission angle is small, $\theta_{q} \simeq k_{\perp} / \omega \ll 1$. The previous discussion implies (the factor of 2 in the equation below is conventional)

$$
\tau_{q} \frac{k_{\perp}}{\omega} \simeq \frac{2}{k_{\perp}} \quad \Longrightarrow \quad \tau_{q} \simeq \frac{2 \omega}{k_{\perp}^{2}} \simeq \frac{2}{\omega \theta_{q}^{2}} .
$$

The above argument is completely general: it holds for gluon emissions in either the medium or the vacuum. What is different, however, is the typical value of $k_{\perp}$ in the two cases.

For emissions in the vacuum, $k_{\perp}$ is a priori arbitrary. However, the associated, bremsstrahlung, spectrum, which takes the familiar form

$$
\omega \frac{\mathrm{d} N^{\mathrm{vac}}}{\mathrm{d} \omega \mathrm{d} k_{\perp}^{2}} \simeq \frac{\alpha_{s} C_{F}}{k_{\perp}^{2}} \simeq \alpha_{s} C_{F} \theta_{q}^{2} \tau_{q}^{2},
$$

is such that large values of $k_{\perp}$ are strongly suppressed, so most of the radiation is quasicollinear with its source $\left(\theta_{q} \rightarrow 0\right)$. The second equality in eq. (2.2), which is clearly true in view of eq. (2.1), has a simple physical interpretation. After the quark is created at $t_{0}=0$, a gluon with energy $\omega$ and transverse momentum $k_{\perp}$ is emitted by a time $t \sim \tau_{q}$ and not much later. The factor $\tau_{q}^{2}$ represents the temporal (or longitudinal) phase-space for such emissions. The factor $\theta_{q}^{2} \simeq v_{\perp}^{2}$ is the square of the emission vertex.

Within a medium, on the other hand, the gluon can acquire an additional transverse momentum via scattering off the medium constituents (see the graphical representation 


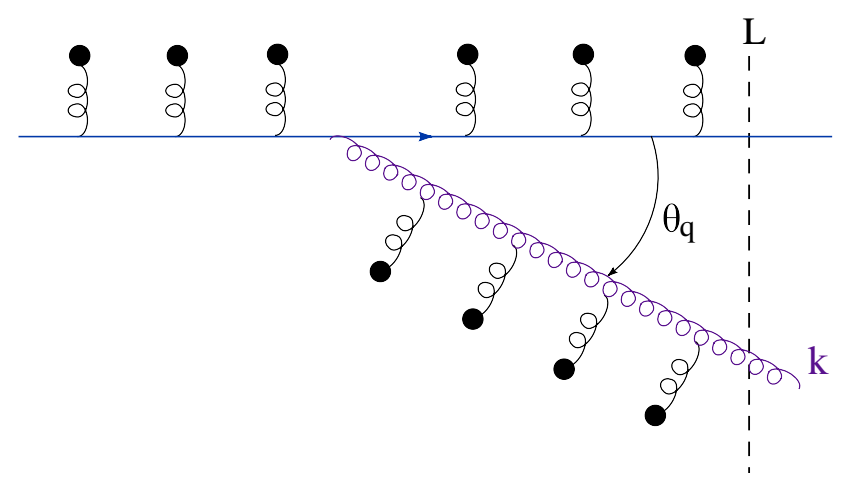

Figure 1. The standard representation of the Feynman graph for medium-induced gluon radiation: both the quark and the emitted gluon undergo multiple scattering off the medium constituents.

in figure 1). If the medium is sufficiently dense, the momentum acquired in this way can be large and then the gluon spectrum is shifted towards a non-zero central value. At weak coupling, one can assume the successive collisions to be independent even when the medium is dense: the gluon mean free path $\ell$ scales like $1 / \alpha_{s}$ and for sufficiently small $\alpha_{s}=g^{2} /(4 \pi)$ it becomes much larger than the screening length $\mu_{D}^{-1} \propto 1 / g$ for charge correlations in the medium ( $\mu_{D}$ is the Debye mass). The gluon receives random kicks from the medium constituents, with each kick transferring a momentum squared $\sim \mu_{D}^{2}$, so its average transverse momentum squared grows at a rate

$$
\frac{\mathrm{d}\left\langle k_{\perp}^{2}\right\rangle}{\mathrm{d} t} \simeq \frac{\mu_{D}^{2}}{\ell} \equiv \hat{q}
$$

The quantity $\hat{q}$ is a local transport coefficient known as the jet quenching parameter.

The interactions within the medium wash out the quantum coherence between the gluon and its source, and thus determine the formation time $\tau_{f}$ for medium-induced emissions. Specifically, within a time $\tau_{f}$, the gluon acquires a transverse momentum squared $k_{f}^{2} \simeq \hat{q} \tau_{f}$ with $\tau_{f}$ related to $k_{f}^{2}$ as shown in eq. (2.1). We thus deduce

$$
k_{f}^{2} \simeq(2 \omega \hat{q})^{1 / 2} \quad \text { and } \quad \tau_{f} \simeq \sqrt{\frac{2 \omega}{\hat{q}}} .
$$

At the time of emission, the gluon has an average transverse momentum $k_{f}$ and hence it makes a typical angle $\theta_{f} \simeq k_{f} / \omega$ (the formation angle).

In order for the gluon to be formed in the medium, one needs $\tau_{f} \leq L$, with $L$ the longitudinal extent of the slice of the medium which is crossed by the quark. Hence, the maximal possible value for $k_{f}$, known as the saturation momentum $Q_{s}$, is given by $Q_{s}^{2}=\hat{q} L$ and is reached for a gluon with a frequency $\omega_{c}$ such that $\tau_{f}\left(\omega_{c}\right)=L$. These relations imply

$$
\omega_{c}=\frac{1}{2} \hat{q} L^{2}, \quad Q_{s}^{2}=\hat{q} L, \quad \theta_{c}=\frac{Q_{s}}{\omega_{c}}=\frac{2}{Q_{s} L}=\frac{2}{\sqrt{\hat{q} L^{3}}} .
$$

$\theta_{c}$ is the formation angle for a gluon with frequency $\omega_{c}$ and is the minimal angle in the problem: gluons with larger frequencies $\omega>\omega_{c}$ and smaller angles $\theta_{q}<\theta_{c}$ cannot be 
emitted via this mechanism. The medium is dense provided $k_{f} \gg \mu_{D}$. In view of eq. (2.3), this requires the in-medium formation time $\tau_{f}$ to be substantially larger than the mean free path $\ell: \tau_{f} \gg \ell \gg \mu_{D}^{-1}$. Given the $\omega$-dependence of the formation time $\tau_{f}$, this last constraint implies a lower limit on the gluon energy: $\omega \gg \omega_{\min }$ with

$$
\omega_{\min } \equiv \frac{1}{2} \ell \mu_{D}^{2}=\frac{1}{2} \hat{q} \ell^{2} .
$$

Since, moreover, $Q_{s}>k_{f}$ and $L>\tau_{f}$, the above relations also imply that the limiting angle $\theta_{c} \sim 1 /\left(Q_{s} L\right)$ is truly small: $\theta_{c} \ll 1$. (Some typical values for heavy ion collisions at RHIC and the LHC are $L=6 \mathrm{fm}$ and $\hat{q}=2 \div 10 \mathrm{GeV}^{2} / \mathrm{fm}$, yielding $\theta_{c}=0.01 \div 0.02$.)

More generally, for a given energy $\omega<\omega_{c}$, the quantities $k_{f}$ and $\theta_{f}$ introduced above - the average transverse momentum and emission angle at the formation time - represent lower limits on the respective kinematical variables in the BDMPS-Z spectrum. For what follows, it is useful to express the quasi-local quantities $k_{f}$ and $\theta_{f}$, which are controlled by the physics at the scale $\tau_{f}$, in terms of the global ( $L$-dependent) quantities in eq. (2.5), which represent their absolute limits for $\tau_{f}=L$ :

$$
\tau_{f}=L \sqrt{\frac{\omega}{\omega_{c}}}, \quad k_{f}=Q_{s}\left(\frac{\omega}{\omega_{c}}\right)^{1 / 4}, \quad \theta_{f} \simeq \frac{k_{f}}{\omega}=\theta_{c}\left(\frac{\omega_{c}}{\omega}\right)^{3 / 4} .
$$

These formulæ make clear that the relatively soft gluons with $\omega \ll \omega_{c}$ are emitted very fast $\left(\tau_{f} \ll L\right)$ and at relatively large angles $\left(\theta_{f} \gg \theta_{c}\right)$. Such gluons are very efficient in broadening the jet energy in the transverse plane.

While the quark propagates though the medium it receives kicks from the medium constituents and it can radiate after any of those kicks. When $\omega \gg \omega_{\min }$, the typical distance $\ell$ between two consecutive kicks is much shorter than the formation time $\tau_{f}$. Accordingly, a large number of scattering centers $N_{\text {coh }} \simeq \tau_{f} / \ell \gg 1$ act coherently as a single source of radiation. This subset of $N_{\text {coh }}$ constituents can be located anywhere inside the medium, meaning that the time $t_{1}$ at which a particular emission is initiated is delocalized within the interval $0<t_{1}<L-\tau_{f}$. Thus, unlike vacuum emissions which start right away after a hard scattering, the medium-induced emissions can be initiated at any point inside the medium. Accordingly, the longitudinal phase-space for medium-induced gluon radiation is $\left(L-\tau_{f}\right) \tau_{f} \sim L \tau_{f}$, which for $\omega \ll \omega_{c}$ is parametrically larger than the corresponding bremsstrahlung phase-space $\tau_{q}^{2}$ (for the same kinematics). We shall later derive the gluon spectrum at the formation time and thus find a Gaussian centered at $k_{f}$ :

$$
\left.\omega \frac{\mathrm{d} N}{\mathrm{~d} \omega \mathrm{d} k_{\perp}^{2}}\right|_{\text {form }} \propto \alpha_{s} C_{F} \theta_{q}^{2} \tau_{f} L \exp \left\{-\frac{k_{\perp}^{2}}{k_{f}^{2}}\right\} .
$$

The prefactor in this expression is similar to that in eq. (2.2): the only difference refers to the replacement $\tau_{q}^{2} \rightarrow \tau_{f} L$ for the longitudinal phase-space. Since $\theta_{q} \propto k_{\perp}$, it is clear that this spectrum is strongly peaked at $k_{\perp}=k_{f}$. Hence, for parametric estimates, one can replace $\theta_{q} \rightarrow \theta_{f}$ in the prefactor. For $k_{\perp} \sim k_{f}$ and $\omega \ll \omega_{c}$, one has $\tau_{q} \sim \tau_{f} \ll L$, hence eq. (2.8) is indeed enhanced w.r.t. the vacuum spectrum (2.2), by the large factor 


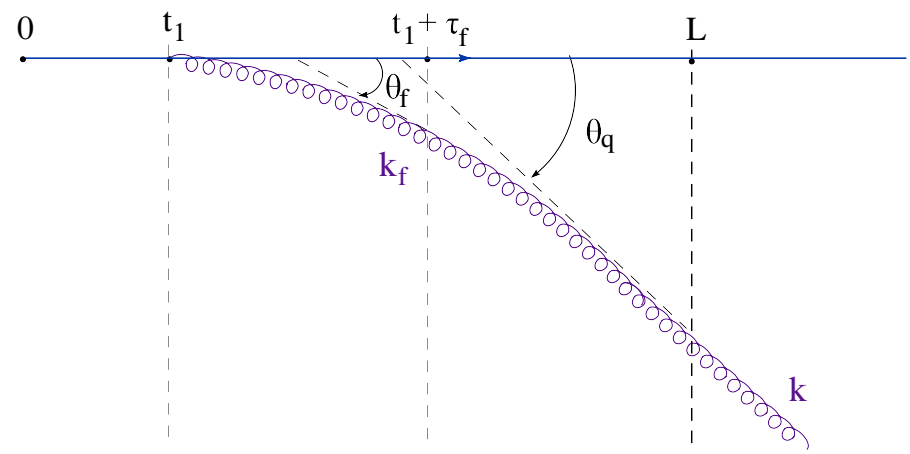

Figure 2. A cartoon illustrating the space-time picture of a medium-induced gluon radiation. The gluon formation is initiated at time $t_{1}$ and terminated at time $t_{1}+\tau_{f}$. The gluon leaves the medium with its final momentum $\boldsymbol{k}$ at time $L$. The interactions with the medium are not explicitly shown.

$L / \tau_{f} \gg 1$. This factor counts the number of times that a medium-induced gluon can be formed inside the medium.

Eq. (2.8) is not yet the final BDMPS-Z spectrum: after being formed, the gluon will still propagate inside the medium over a distance $L-\tau_{f}-t_{1}$ and thus acquire an additional momentum broadening $\Delta k_{\perp}^{2} \simeq \hat{q}\left(L-\tau_{f}-t_{1}\right)$. Accordingly, its final momentum $k_{\perp}^{2}=k_{f}^{2}+\Delta k_{\perp}^{2}$ can take any value between $k_{f}^{2}=\hat{q} \tau_{f}$ and $Q_{s}^{2}=\hat{q} L$. This results in the following kinematics domain for the final gluon, as would be measured by a detector:

$$
\omega_{\min } \lesssim \omega \lesssim \omega_{c}, \quad k_{f} \lesssim k_{\perp} \lesssim Q_{s}
$$

Within this range in $k_{\perp}$, the BDMPS-Z distribution is roughly flat (see eq. (4.22) below).

In discussing interference phenomena in what follows, it will be more convenient to use angular variables instead of transverse momenta. Using eq. (2.9), one immediately finds the following range for the final gluon angle $\theta_{q} \simeq k_{\perp} / \omega$ :

$$
\theta_{f}=\theta_{c}\left(\frac{\omega_{c}}{\omega}\right)^{3 / 4} \lesssim \theta_{q} \lesssim \theta_{s}=\theta_{c} \frac{\omega_{c}}{\omega}
$$

where $\theta_{s} \simeq Q_{s} / \omega$. The space-time picture of medium-induced radiation is illustrated in figure 2 .

\subsection{Qualitative discussion of interference}

To study interference effects, we shall replace the quark probe considered in the previous subsection with a color dipole, that is a quark $(q)$ and antiquark $(\bar{q})$ in a color singlet state which separate from each other at constant velocities which make a relative angle $\theta_{q \bar{q}}$ (the dipole opening angle), which is relatively small: $\theta_{q \bar{q}} \ll 1$. The two fermions are massless, so they propagate at the speed of light. The dipole is created at time $t_{0}=0$ and for later times its transverse size grows like $r_{\perp}(t) \simeq \theta_{q \bar{q}} t$. By 'transverse' we here mean the direction perpendicular to the common direction of motion of the quark and the antiquark (the 'longitudinal axis'), as defined by the trajectory of their center of mass. Interference occurs if the transverse wavelength of the gluon which is about to be emitted 

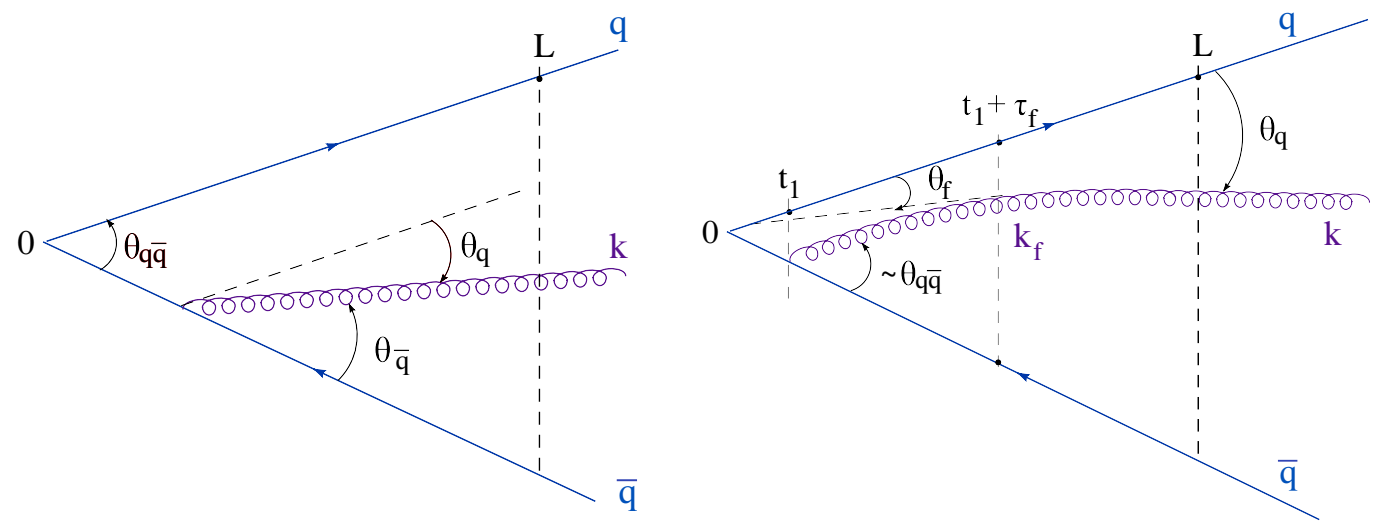

Figure 3. Gluon emission by the dipole. Left: the geometry of the final state. Right: the spacetime picture of a typical emission contributing to interference in the case $\theta_{q \bar{q}}>\theta_{f}$. When the emission is initiated, at time $t_{1} \sim \tau_{\text {int }}$ by the antiquark, the virtual gluon is co-moving with the quark. When the emission is completed, at time $t_{1}+\tau_{f}$, the gluon makes an angle $\theta_{f}$ with the quark.

is large enough for the gluon to have an overlap with both sources. When this happens, the gluon 'sees' the overall color charge of the $q \bar{q}$ pair, which is zero, so it is not emitted anymore (destructive interference).

For vacuum radiation, the interference effects are well known to lead to angular ordering $[28,29]$. The distance between the quark and the antiquark at the time of emission is $\sim \theta_{q \bar{q}} \tau_{q}$ with $\tau_{q}=2 \omega / k_{\perp}^{2}$. Interference occurs if this distance is smaller than the transverse wavelength $\lambda_{\perp} \simeq 1 / k_{\perp}$ of the gluon. Using $k_{\perp} \simeq \omega \theta_{q}$, with $\theta_{q}$ the emission angle, we deduce

$$
\theta_{q \bar{q}} \frac{1}{\omega \theta_{q}^{2}} \lesssim \frac{1}{\omega \theta_{q}} \quad \Longrightarrow \quad \theta_{q} \gtrsim \theta_{q \bar{q}}
$$

That is, the interference is important only for emissions at large angles, outside the dipole cone.

The interference effects can be also discussed at the level of the bremsstrahlung spectrum produced by the dipole. This is given by the following generalization of eq. (2.2)

$$
\omega \frac{\mathrm{d} N_{\mathrm{dip}}^{\mathrm{vac}}}{\mathrm{d}^{3} \boldsymbol{k}} \simeq \alpha_{s} C_{F}\left(\theta_{q} \tau_{q}-\theta_{\bar{q}} \tau_{\bar{q}}\right)^{2},
$$

with $k^{\mu}=(\omega, \boldsymbol{k})$ the 4 -vector of the emitted gluon, $\tau_{q} \simeq 2 /\left(\omega \theta_{q}^{2}\right)$ and $\tau_{\bar{q}} \simeq 2 /\left(\omega \theta_{\bar{q}}^{2}\right)$. (Note that our sign conventions for the emission angles are such that $\theta_{q}<0$ and $\theta_{\bar{q}}>0$ for emissions inside the dipole and $\theta_{q} \theta_{\bar{q}}>0$ for emissions outside the dipole; see also figure 3 left. With this convention, one has $\theta_{q \bar{q}}=\theta_{\bar{q}}-\theta_{q}>0$. Also, when using an angle within a parametric estimate or an inequality, we always mean its absolute value.) By expanding the square in the r.h.s. one generates the direct emission terms, from the quark $\left(\theta_{q}^{2} \tau_{q}^{2}\right)$ and respectively the antiquark $\left(\theta_{\bar{q}}^{2} \tau_{\bar{q}}^{2}\right)$, together with the interference term $-2 \theta_{q} \theta_{\bar{q}} \tau_{q} \tau_{\bar{q}}$, which has an overall minus sign because the two sources have opposite charges. Inside the dipole cone, where $\theta_{q} \theta_{\bar{q}}<0$, the interference term is relatively unimportant: the radiation is strongly peaked around the direction of the quark $\left(\theta_{q} \approx 0\right)$ or of the antiquark $\left(\theta_{\bar{q}} \approx 0\right)$, 
where it is dominated by the respective direct emission. However for large emission angles $\theta_{q}, \theta_{\bar{q}} \gg \theta_{q \bar{q}}$ one has $\theta_{q} \simeq \theta_{\bar{q}}$ and $\tau_{q} \simeq \tau_{\bar{q}}$ and then the total radiation vanishes: the direct emissions are compensated by the interference term.

We now turn to the description of the medium-induced gluon radiation from an energetic $q \bar{q}$ dipole created in the medium. It is intuitively clear that, if the dipole angle is sufficiently large (larger than the maximal emission angle $\theta_{s}$ introduced in the previous subsection, cf. eq. (2.10)), the radiation patterns produced by the quark and the antiquark via interactions in the medium have no overlap with each other and thus they are independent. The question we would like to address is what happens when the dipole opening angle is not that large. In that case, and in view of the experience with radiation in the vacuum, one may expect the dipole antenna pattern to be affected by interference effects between the emissions by the quark and the antiquark. However, as we shall now argue, this expectation is generally incorrect: for a sufficiently dense medium and a dipole angle $\theta_{q \bar{q}}$ which is not very small (see below for the precise condition), only those gluons which are emitted very close to the $q \bar{q}$ vertex can be coherent with both emitters and thus lead to interference. Accordingly, the interference effects are parametrically suppressed as compared to the direct emissions from each of the quarks.

The requirement that the two sources $(q$ and $\bar{q})$ be coherent with each other has two aspects:

(i) Quantum coherence. The emission process preserves the quantum coherence of the $q \bar{q}$ system so long as the virtual gluon overlaps with both sources in the course of formation. (After formation, the gluon rescattering in the medium can change its momentum but cannot affect the interference process since the gluon is already decorrelated from its sources.) For that to be possible, the transverse wavelength $\lambda_{f}=1 / k_{f}$ of the gluon at the time of formation should be larger than the typical $q \bar{q}$ distance around that time. For emissions in the vacuum, this condition immediately leads to angular ordering, as discussed around eq. (2.11). But for the medium-induced emissions, the situation is more subtle: during the gluon formation, the transverse size of the $q \bar{q}$ system increases from $r_{\min } \simeq \theta_{q \bar{q}} t_{1}$ to $r_{\max } \simeq \theta_{q \bar{q}} t_{2}$, where $t_{1}$ is the time when the emission is initiated and $t_{2}=t_{1}+\tau_{f}$. Our calculations in section 5 and in appendix A (see eqs. (5.12) and (A.7)) show that the relevant $q \bar{q}$ size to be compared to $\lambda_{f}$ is the geometric average of these two extreme scales. This reflects the diffusive nature of the gluon dynamics during formation. That is, the condition of quantum coherence amounts to $\sqrt{r_{\min } r_{\max }} \lesssim \lambda_{f}$, or

$$
\sqrt{t_{1}\left(t_{1}+\tau_{f}\right)} \lesssim \frac{\lambda_{f}}{\theta_{q \bar{q}}} \equiv \tau_{\lambda}
$$

The scale $\tau_{\lambda}$ introduced above will be referred to as the transverse resolution time. Using $\lambda_{f}=1 / k_{f}$ with $k_{f}$ given by eq. (2.4), it is easy to see that

$$
\tau_{\lambda}=\frac{1}{\theta_{q \bar{q}}(\hat{q} \omega)^{1 / 4}}=\tau_{f} \frac{\theta_{f}}{\theta_{q \bar{q}}},
$$

where the second estimate follows since $k_{f} \simeq \omega \theta_{f}$ and $\tau_{f} \simeq 2 /\left(\omega \theta_{f}^{2}\right)$. 
Eq. (2.13) implies an upper limit on $t_{1}$ that we shall now explicitly work out. It is first of all clear that $t_{1}<\tau_{\lambda}$. But the actual constraint on $t_{1}$ can be even more restrictive, depending upon the ratio $\theta_{q \bar{q}} / \theta_{f}$ [cf. eq. (2.14)]:

(i.a) For relatively small dipole angles $\theta_{q \bar{q}} \ll \theta_{f}$, one has $\tau_{\lambda} \gg \tau_{f}$ and then eq. (2.13) implies $t_{1} \lesssim \tau_{\lambda}$. In this regime, the interference occurs between the typical BDMPS-Z spectra of the two emitters, which during the formation process are localized at angles $\sim \theta_{f} \gg \theta_{q \bar{q}}$ around their respective sources: if initiated at a time $t_{1} \lesssim \tau_{\lambda}$, the medium-induced emissions by the quark and respectively the antiquark will overlap with each other and thus interfere.

(i.b) For larger dipole angles $\theta_{q \bar{q}} \gg \theta_{f}$, one has $\tau_{\lambda} \ll \tau_{f}$ and therefore $t_{1} \ll \tau_{f}$ as well. Then eq. (2.13) simplifies to

$$
t_{1} \lesssim \frac{\tau_{\lambda}^{2}}{\tau_{f}} \equiv \tau_{\text {int }}
$$

which introduces a new temporal scale - the interference time $\tau_{\text {int }}$ - which in this regime is much smaller than $\tau_{\lambda}$. This new scale can be rewritten as

$$
\tau_{\text {int }}=\frac{2}{\omega \theta_{q \bar{q}}^{2}}=\tau_{f}\left(\frac{\theta_{f}}{\theta_{q \bar{q}}}\right)^{2},
$$

which is recognized as the vacuum-like formation time for a gluon emitted at an angle $\sim \theta_{q \bar{q}}$. The emergence of this scale is quite natural: in order to overlap with both sources, the gluon must be emitted at a relatively large angle, of order $\theta_{q \bar{q}}$, with respect to its parent fermion. Since $\theta_{q \bar{q}} \gg \theta_{f}$, it is clear that such an emission cannot be triggered by medium interactions; rather, it occurs like in the vacuum, with formation time (2.16). But such a vacuum-like emission by one fermion can interfere with a medium-induced emission by the other fermion and thus contribute to the BDMPS-Z spectrum of the dipole. Indeed, a gluon emitted at an angle $\sim \theta_{q \bar{q}}$, say, by the antiquark will be co-moving with the quark and hence it will behave in the same way as a typical gluon from the quark wavefunction. That is, it will decohere from the quark via medium rescattering and eventually emerge at an angle $\sim \theta_{f}$ w.r.t. the quark (see figure 3 right). Thus, in this regime, one can speak of vacuum-medium interferences.

(ii) Color coherence. In the vacuum, the color state of the dipole is conserved until a gluon emission takes place and the interference pattern is governed solely by quantum coherence. In the medium, the interactions with the medium change the color of each of the propagating parton, via 'color rotation'. For an energetic parton, this rotation amounts to multiplying its wavefunction by a $\mathrm{SU}\left(N_{c}\right)$ matrix-valued phase (a Wilson line) which involves the random color field generated by the constituents of the medium evaluated along the parton trajectory.

For the $q \bar{q}$ pair we have two such Wilson lines, one for each fermion. The color coherence is measured by the 2-point correlation function of these Wilson lines, as 
obtained after averaging over the fluctuations of the background field. Within the 'multiple soft scattering approximation', this 2-point function can be computed to all orders in the medium effects (see eq. (3.27) and eq. (5.3)). As discussed around eq. (5.4), the quark and the antiquark lose any trace of their original color correlation after the decoherence time

$$
\tau_{\mathrm{coh}}=\frac{2}{\left(\hat{q} \theta_{q \bar{q}}^{2}\right)^{1 / 3}}=\tau_{f}\left(\frac{\theta_{f}}{\theta_{q \bar{q}}}\right)^{2 / 3} .
$$

Accordingly, interference effects are possible only for the gluon emissions initiated at a time $t_{1}$ smaller than $\tau_{\text {coh }}$. This scale $\tau_{\text {coh }}$ can be related to $L$ by using eqs. (2.17) and (2.7):

$$
\tau_{\mathrm{coh}} \simeq\left(\frac{\theta_{c}}{\theta_{q \bar{q}}}\right)^{2 / 3} L .
$$

Quite remarkably, this estimate involves the limiting angle $\theta_{c}$ of the BDMPS-Z spectrum, although the present physical context is quite different: the scale $\tau_{\text {coh }}$ refers to the color coherence between the two emitters independently of their radiation.

The previous discussion shows that the study of interference effects for the mediuminduced dipole radiation is a multi-scale problem. While the details of the in-medium dipole antenna pattern are expected to depend upon all these scales, the phase-space for interference is controlled by the smallest of them, $\tau_{\min }=\min \left(\tau_{\lambda}, \tau_{\text {int }}, \tau_{\text {coh }}\right)$. As a consequence, the interference contribution to the gluon spectrum (that is, the contribution of diagrams in which the gluon is emitted by the quark in the amplitude and by the antiquark in the complex conjugate amplitude, or vice versa), does not scale with the medium length $L$, but with $\tau_{\min }$.

Remarkably, eqs. (2.14), (2.16) and (2.17), which can be summarized as

$$
\tau_{f} \sim \tau_{\text {coh }}\left(\frac{\theta_{q \bar{q}}}{\theta_{f}}\right)^{2 / 3} \sim \tau_{\lambda} \frac{\theta_{q \bar{q}}}{\theta_{f}} \sim \tau_{\text {int }}\left(\frac{\theta_{q \bar{q}}}{\theta_{f}}\right)^{2} \sim L \sqrt{\frac{\omega}{\omega_{c}}},
$$

show that, for a given in-medium formation time $\tau_{f}$, all the three time scales relevant for coherence depend solely upon the ratio $\theta_{q \bar{q}} / \theta_{f}$. Physically, this is a consequence of the fact that, at formation, the medium-induced gluon distribution (2.8) is characterized by the formation angle $\theta_{f}$ alone (for a given $\tau_{f}$ ). Hence, when discussing interference effects, it is natural to compare $\theta_{q \bar{q}}$ to $\theta_{f}$. In addition, as we shall see, there is a change of regime when $\tau_{\text {coh }}$ becomes as large as the medium size $L$, which according to eq. (2.18) happens when $\theta_{q \bar{q}} \simeq \theta_{c}$. We are thus led to consider the following three ranges for $\theta_{q \bar{q}}$ :

1. Relatively large dipole angles, $\theta_{f} \lesssim \theta_{q \bar{q}} \lesssim \theta_{s}$. In this regime eqs. (2.14), (2.16) and (2.17) imply the following hierarchy of scales

$$
\tau_{\text {int }} \lesssim \tau_{\lambda} \lesssim \tau_{\text {coh }} \lesssim \tau_{f} \quad \text { when } \quad \theta_{q \bar{q}} \gtrsim \theta_{f},
$$

which shows that, for such large dipole angles, the condition (2.15) of quantum coherence is the most restrictive one. Accordingly, in this regime, the longitudinal 
phase-space for interferences is of order $\sim \tau_{\text {int }} \tau_{f}$ and thus is suppressed with respect to the corresponding phase-space $\sim \tau_{f} L$ for direct emissions by each of the two sources by a factor

$$
\mathcal{R}=\frac{\tau_{\text {int }}}{L} \sim \sqrt{\frac{\omega}{\omega_{c}}}\left(\frac{\theta_{f}}{\theta_{q \bar{q}}}\right)^{2} \ll 1 .
$$

The range of values spanned by $\mathcal{R}$ within this regime is displayed in eq. (5.17) below.

2. Relatively small dipole angles $\theta_{c} \ll \theta_{q \bar{q}} \ll \theta_{f}$. In this case, the strongest limitation on the phase-space for interference comes from the requirement of color coherence, as clear from the fact that the ordering of time scales is now reverted:

$$
\tau_{f} \ll \tau_{\text {coh }} \ll \tau_{\lambda} \ll \tau_{\text {int }} \quad \text { when } \quad \theta_{q \bar{q}} \ll \theta_{f} .
$$

So, the longitudinal phase-space for interference is now of order $\tau_{\operatorname{coh}} \tau_{f}$. So long as $\theta_{q \bar{q}} \gg \theta_{c}$, this is still strongly suppressed as compared to the phase-space $\sim \tau_{f} L$ for direct emissions, as manifest from eq. (2.18). Hence, in this regime too, the interference contribution to the spectrum is parametrically small (see also eq. (5.18) for the corresponding range):

$$
\mathcal{R}=\frac{\tau_{\mathrm{coh}}}{L}=\left(\frac{\theta_{c}}{\theta_{q \bar{q}}}\right)^{2 / 3} \ll 1 .
$$

Note that, in this case, the medium-induced radiation by the dipole (the incoherent sum of the two corresponding spectra by the quark and the antiquark) is distributed at large angles $\theta_{q} \simeq \theta_{\bar{q}} \gtrsim \theta_{f} \gg \theta_{q \bar{q}}$, that is, well outside the dipole cone. One may wonder why the total radiation in that case is not simply zero (as it would be for the large angle radiation by a color-singlet dipole in the vacuum). The reason is that, so long as $\theta_{q \bar{q}} \gg \theta_{c}$, a $q \bar{q}$ pair immersed in the medium is not a 'color singlet' anymore, except for a very brief period of time $\tau_{\text {coh }} \ll L$.

3. Very small dipoles angles $\theta_{q \bar{q}} \lesssim \theta_{c}$. When the dipole angle is even smaller, $\theta_{q \bar{q}} \lesssim \theta_{c}$, the color coherence time $\tau_{\text {coh }}$ becomes as large as the medium size $L$, cf. eq. (2.18). Then, the $q \bar{q}$ pair preserves its color and quantum coherence throughout the medium, so the interference effects are not suppressed anymore and they act towards reducing the medium-induced radiation by the dipole. For sufficiently small angles $\theta_{q \bar{q}} \ll \theta_{c}$, the color decoherence is parametrically small and the total (in-medium) radiation becomes negligible: the interference effects and the direct emissions nearly compensate each other. ${ }^{2}$ This conclusion is in agreement ${ }^{3}$ with the results in [26], obtained by working to leading order in the 'opacity expansion' [13] (the single scattering approximation).

In summary, we have argued that for sufficiently large dipole angles $\theta_{q \bar{q}} \gg \theta_{c}$, the interference effects for the medium-induced radiation are negligible, so the total BDMPS-Z

\footnotetext{
${ }^{2}$ The net result should be of order $\left(\theta_{q \bar{q}} / \theta_{c}\right)^{2}$, as clear by inspection of eq. (3.29) below.

${ }^{3}$ We would like to thank Carlos Salgado for useful discussions on this point.
} 
spectrum by the dipole is the incoherent sum of the respective spectra produced by the quark and the antiquark. For smaller angles $\theta_{q \bar{q}} \lesssim \theta_{c}$, the interference effects are not suppressed anymore and they eventually cancel the direct emissions when $\theta_{q \bar{q}} \ll \theta_{c}$. The transition between the two regimes, occurring at $\theta_{q \bar{q}} \simeq \theta_{c}$, could in principle be studied within the formalism that we shall develop later. However, such a study goes beyond the approximation schemes that we shall use throughout this paper and which are adapted to the most interesting regime at $\theta_{q \bar{q}} \gg \theta_{c}$.

Note that, although so far we have focused on gluons with relatively soft energies, $\omega \ll \omega_{c}$, our main conclusions remain valid when $\omega$ approaches the limiting value $\omega_{c}$, as we now argue. When $\omega \sim \omega_{c}$, one has $\tau_{f} \sim L$ and $\theta_{f} \sim \theta_{c}$, so the intermediate regime of 'relatively small dipole angles' disappears. Yet, eq. (2.21) implies that, so long as $\theta_{q \bar{q}} \gg \theta_{f}\left(\omega_{c}\right)=\theta_{c}$, the interference effects are relatively small even for $\omega \sim \omega_{c}$. This is so because the time scale $\tau_{\text {int }}$ which limits quantum coherence is still much smaller than $L$ in this regime.

We conclude our discussion by observing that the emission of BDMPS-Z-like gluons is not the only medium-induced radiation by the dipole. Indeed, in ref. [27], it has been shown that, as a consequence of color decoherence in a dense medium, the dipole produces additional, soft, gluon radiation, which is emitted outside the medium $\left(t_{1} \gtrsim L\right)$ and also outside the dipole cone $\left(\theta_{q}, \theta_{\bar{q}}>\theta_{q \bar{q}}\right)$. As we shall explain in sections 3.2 and 6 , this alternative mechanism operates only for relatively small dipole angles $\theta_{q \bar{q}} \ll \theta_{f}$ and the associated radiation is mostly collinear with the $q \bar{q}$ axis. Moreover, the associated gluon spectrum is simply the bremsstrahlung spectrum and hence it is independent of the medium size $L$. On the contrary, the medium-induced radiation that we consider is emitted at larger angles, within a range $\theta_{f} \lesssim \theta_{q} \lesssim \theta_{s}$, and it has a strength proportional to the medium length. Hence, these two mechanisms, which are simultaneously present in the medium, lead to gluon spectra with very small overlap.

\section{General set-up and formalism}

In this section, we shall more precisely describe our physical problem - a color dipole which radiates gluons while propagating through a QCD medium (say, a quark-gluon plasma) and the formalism to be used for its study. As noticed in the Introduction, a similar set-up has been also used in refs. [26, 27]. But the focus there was on some special physical conditions, allowing for additional simplifications: the single scattering approximation ('dilute medium') in [26] and the restriction to out-of-medium emissions ('soft and collinear gluons') in [27]. Here, we shall keep our discussion as general as possible, in such a way to encompass the physics of medium-induced gluon radiation in the multiple soft scattering regime. In the process, we shall also make contact with the results in ref. [27] and clarify their applicability.

\subsection{The amplitude for gluon emission}

The in-medium dipole dynamics will be treated in the semi-classical approximation, that is, by solving Yang-Mills equations in which the dipole enters as a classical source of 
color charge. The medium rescattering will be resummed to all orders via a background field method. The effects of this rescattering on the quark and the antiquark legs of the dipole will be treated in the eikonal approximation. The corresponding effects on the emitted gluon will be treated exactly (within the semi-classical approximation), by using an appropriate background field propagator. The background field is assumed to be random, with a Gaussian distribution, and the average over its fluctuations will be performed using techniques borrowed from the color glass condensate [30]. The underlying assumptions are that the two quarks are very energetic, with momenta much larger than any momentum which can be transferred by the medium, whereas the emitted gluon carries (transverse) momenta comparable to those of the medium. Under these assumptions, our calculations are correct to lowest order in the color charge of the dipole but to all orders in the medium effects. This formalism has been used in ref. [33] to study the radiation by a single quark and shown to encompass the essential BDMPS-Z physics.

The color dipole is a pair of classical, massless, particles with opposite color charges (so that the pair is a color singlet) which is produced at time $t_{0}=0$ by some hard process occurring inside the medium. After being produced, the two particles separate from each other at constant velocities, $\boldsymbol{u}=\boldsymbol{p}_{q} / E_{q}$ for the quark $(q)$ and $\overline{\boldsymbol{u}}=\boldsymbol{p}_{\bar{q}} / E_{\bar{q}}$ for the antiquark $(\bar{q})$, which make a relative angle $\theta_{q \bar{q}}: \boldsymbol{u} \cdot \overline{\boldsymbol{u}}=\cos \theta_{q \bar{q}}$. Here $\boldsymbol{p}_{q}$ and $E_{q}=\left|\boldsymbol{p}_{q}\right|$ are the 3-momentum and the energy of the quark (and similarly for the antiquark), assumed to much larger than any other scale in the problem. We choose the longitudinal axis $\left(x^{3}\right)$ as the direction of motion of the center-of-mass of the $q \bar{q}$ pair. In the medium rest frame, the dipole has a relatively large longitudinal boost $\gamma \gg 1$ and hence a small opening angle $\theta_{q \bar{q}} \sim 1 / \gamma$. This angle will be nevertheless assumed to be significantly larger than the critical angle for medium-induced radiation $\theta_{c} \sim 1 /\left(Q_{s} L\right)$, which is very small $\left(\theta_{c} \ll 1\right)$ as previously explained. The dipole propagates through the medium along a longitudinal distance $L$ before escaping into the vacuum.

The $Q C D$ medium is described as a random color background field $\mathcal{A}_{a}^{\mu}$ with a Gaussian distribution. As well known e.g. from the experience with the color glass condensate [30], this description becomes simpler by working in a Lorentz frame in which the medium is strongly boosted (an 'infinite momentum frame'). For the problem at hand, it is convenient to choose the 'dipole frame' in which the COM of the $q \bar{q}$ pair is nearly at rest, meaning that the plasma is boosted (essentially, by the dipole $\gamma$ factor introduced above) in the negative $x^{3}$ direction. In this new frame and in light-cone (LC) gauge $A_{a}^{+}=0$, the background field has only one non-trivial component, $\mathcal{A}_{a}^{\mu}=\delta^{\mu-} \mathcal{A}_{a}^{-}$, which is moreover independent of the LC 'time' $x^{-}$, by Lorentz time dilation. We have introduced here the LC components of the 4 -vector $\mathcal{A}_{a}^{\mu}$, defined in the standard way; e.g. $x^{\mu}=\left(x^{+}, x^{-}, \boldsymbol{x}_{\perp}\right)$, with

$$
x^{+}=\frac{1}{\sqrt{2}}\left(x^{0}+x^{3}\right), \quad x^{-}=\frac{1}{\sqrt{2}}\left(x^{0}-x^{3}\right), \quad \boldsymbol{x}_{\perp}=\left(x^{1}, x^{2}\right) .
$$

In the dipole frame, both the dipole angle $\theta_{q \bar{q}}$ and the characteristic medium angle $\theta_{c}$ are enhanced by a factor $\gamma$ (so, in particular, $\theta_{q \bar{q}} \sim \mathcal{O}(1)$ ), but the inequality $\theta_{q \bar{q}} \gg \theta_{c}$ remains of course true. In view of that, and in order to avoid a proliferation of symbols, we shall use the same notations for quantities in the plasma rest frame and in the dipole frame - 
the difference should be clear from the context. Moreover, we shall often use the smallangle version of a parametric estimate (e.g., $\tau_{q} \simeq 2 / \omega \theta_{q}^{2}$ ) even when working in the boosted frame, where the angles are not necessarily small; what we truly mean by such a writing is an estimate which becomes true after boosting back to the plasma rest frame.

Another advantage of using the dipole frame refers to the correlations between the charged constituents of the medium: the longitudinal $\left(x^{+}\right)$range of the correlations, which was $1 / \mu_{D}$ in the original frame, is now Lorentz-contracted to $1 /\left(\gamma \mu_{D}\right)$. When probing this distribution over relatively large longitudinal separations $\Delta x^{+} \gg 1 /\left(\gamma \mu_{D}\right)$, one can describe the medium constituents as independent color charges with a current density $J_{\text {med }}^{\mu, a}(x)=\delta^{\mu-} \rho^{a}\left(x^{+}, \boldsymbol{x}_{\perp}\right)$ and a local 2-point correlation

$$
\left\langle\rho^{a}\left(x^{+}, \boldsymbol{x}_{\perp}\right) \rho^{b}\left(y^{+}, \boldsymbol{y}_{\perp}\right)\right\rangle=\delta^{a b} \delta\left(x^{+}-y^{+}\right) \delta^{(2)}\left(\boldsymbol{x}_{\perp}-\boldsymbol{y}_{\perp}\right) n_{0},
$$

where $n_{0}$ is the average color charge squared per unit volume, assumed to be homogeneous. (For a longitudinally expanding medium, this would be a function of $x^{+}$.) If the medium is a weakly coupled quark-gluon plasma (QGP), then $n_{0} \propto \gamma T \mu_{D}^{2}$ in the dipole frame. Such a color charge distribution gives rise to the following distribution for the background field $\mathcal{A}_{a}^{-}$:

$$
\left\langle\mathcal{A}_{a}^{-}\left(x^{+}, \boldsymbol{q}_{\perp}\right) \mathcal{A}_{b}^{-}\left(y^{+}, \boldsymbol{k}_{\perp}\right)\right\rangle=\delta_{a b} \delta\left(x^{+}-y^{+}\right)(2 \pi)^{2} \delta^{(2)}\left(\boldsymbol{q}_{\perp}-\boldsymbol{k}_{\perp}\right) \frac{n_{0}}{\left(q_{\perp}^{2}+\mu_{D}^{2}\right)^{2}} .
$$

The Debye screening has been heuristically implemented as a 'gluon mass' $\mu_{D}$, although the actual mechanisms at work are generally more complicated.

The gluon radiation by the dipole will be described in the classical approximation, as the additional color field (on top of the background field) generated by the $q \bar{q}$ pair. The classical approximation is correct when the gluon is soft relative to its sources, meaning that $\omega=|\boldsymbol{k}| \ll E_{q}, E_{\bar{q}}$. The color field $a_{a}^{\mu}$ describing the radiation is a small perturbation of the background field and will be obtained by solving the linearized version of the YangMills equation for the total field $A^{\mu}=\delta^{\mu-} \mathcal{A}^{-}+a^{\mu}$ (color indices will be often kept implicit in what follows)

$$
D_{\nu} F^{\nu \mu}=\delta^{\mu-} \rho+J_{\text {dip }}^{\mu}
$$

The dipole color current $J_{\text {dip }}^{\mu}=J_{q}^{\mu}+J_{\bar{q}}^{\mu}$ involves contributions from the quark and the antiquark and also depends upon the background field, because it obeys a covariant conservation law: $\mathcal{D}_{\mu} J_{\text {dip }}^{\mu}=0$, where $\mathcal{D}^{\mu}=\partial^{\mu}+\delta^{\mu-} i g \mathcal{A}^{-}$.

In the vacuum $\left(\mathcal{A}^{-}=0\right)$, the color current associated with a pair of classical particles with constant velocities can be written as $j_{\text {dip }}^{\mu}=j_{q}^{\mu}+j_{\bar{q}}^{\mu}$, with

$$
\begin{aligned}
& j_{q, a}^{\mu}(x)=g u^{\mu} \theta\left(x^{+}\right) \delta\left(x^{-}-u^{-} x^{+}\right) \delta^{(2)}\left(\boldsymbol{x}_{\perp}-\boldsymbol{u}_{\perp} x^{+}\right) \mathcal{C}_{a}, \\
& j_{\bar{q}, a}^{\mu}(x)=-g \bar{u}^{\mu} \theta\left(x^{+}\right) \delta\left(x^{-}-\bar{u}^{-} x^{+}\right) \delta^{(2)}\left(\boldsymbol{x}_{\perp}-\overline{\boldsymbol{u}}_{\perp} x^{+}\right) \mathcal{C}_{a} .
\end{aligned}
$$

We have used here LC notations, with $u^{\mu} \equiv p_{q}^{\mu} / p_{q}^{+}=\left(1, u^{-}, \boldsymbol{u}_{\perp}\right)$ and $\bar{u}^{\mu} \equiv p_{\bar{q}}^{\mu} / p_{\bar{q}}^{+}=$ $\left(1, \bar{u}^{-}, \overline{\boldsymbol{u}}_{\perp}\right)$. Note that for the right-moving dipole, $x^{+}$plays the role of 'time' while $x^{-}$is the 'longitudinal coordinate'. The 'color charges' $\mathcal{C}_{a}$ are the components of a color vector in the adjoint representation describing the orientation of the quark current in the internal 
$\mathrm{SU}\left(N_{c}\right)$ space; for the antiquark, $\overline{\mathcal{C}}_{a}=-\mathcal{C}_{a}$. The current is conserved, $\partial_{\mu} j_{\text {dip }}^{\mu}=0$, since $\partial_{\mu} j_{q, a}^{\mu}=g \mathcal{C}_{a} \delta^{(4)}(x)=-\partial_{\mu} j_{\bar{q}, a}^{\mu}$

In the eikonal approximation, the effect of the medium on the dipole consists merely in color rotations, separately for the quark and the antiquark:

$$
J_{q, a}^{\mu}(x)=\mathcal{U}_{q}^{a b}\left(x^{+}, 0\right) j_{q, a}^{\mu}(x), \quad J_{\bar{q}, a}^{\mu}(x)=\mathcal{U}_{\bar{q}}^{a b}\left(x^{+}, 0\right) j_{\bar{q}, a}^{\mu}(x) .
$$

$\mathcal{U}_{q}\left(x^{+}, 0\right)$ is a Wilson line in the adjoint representation, which is the special case of

$$
\mathcal{U}\left(x^{+}, y^{+} ;\left[\boldsymbol{r}_{\perp}\right]\right)=\mathrm{P} \exp \left\{-i g \int_{y^{+}}^{x^{+}} \mathrm{d} z^{+} \mathcal{A}^{-}\left(z^{+}, \boldsymbol{r}_{\perp}\left(z^{+}\right)\right)\right\}
$$

for $y^{+}=0$ and the transverse path $\boldsymbol{r}_{\perp}\left(z^{+}\right)=\boldsymbol{u}_{\perp} z^{+}$(the quark trajectory in the transverse plane). In eq. (3.7), $\mathcal{A}^{-}=\mathcal{A}_{a}^{-} T^{a}$ is a color matrix in the adjoint representation and the symbol $\mathrm{P}$ denotes time-ordering in $z^{+}$. Using $\left(\partial / \partial x^{+}\right) \mathcal{U}_{q}^{a b}=-i g \mathcal{A}_{a c}^{-}(x) \mathcal{U}_{q}^{c b}$, it is easy to check that $\mathcal{D}_{\mu} J_{q, a}^{\mu}=g \mathcal{C}_{a} \delta^{(4)}(x)=-\mathcal{D}_{\mu} J_{\bar{q}, a}^{\mu}$, so the dipole current is covariantly conserved, as it should. Note that, although the wavefunction of a physical quark is known to transform according to the fundamental representation of the color group, the corresponding color current (3.6) involves a Wilson line in the adjoint representation, since this current is a vector in the color space $\mathrm{SU}\left(N_{c}\right)$. The only trace of the underlying fundamental representation lies in the normalization of the color vector $\mathcal{C}_{a}$, namely $\mathcal{C}_{a} \mathcal{C}_{a}=C_{F}=\left(N_{c}^{2}-1\right) / 2 N_{c}$.

In the LC gauge $A^{+}=0$, only the transverse components $a^{i}$ (with $i=1,2$ ) of the radiated field contribute to the matrix element for gluon emission (see below). The corresponding, linearized, equation of motion is readily obtained from eq. (3.4) and reads

$$
\left(2 \partial^{+} \mathcal{D}^{-}-\nabla_{\perp}^{2}\right) a^{i}=J_{\text {dip }}^{i}-\frac{\partial^{i}}{\partial^{+}} J_{\text {dip }}^{+} .
$$

This equation can be formally solved in terms of the background field Klein-Gordon propagator, i.e. the Green's function for the differential operator in the left hand side. The corresponding solution is well known in the literature (see e.g. [33]) and will be succinctly described here. Given that the background field is independent of $x^{-}$, it is convenient to first perform a Fourier transform to the $k^{+}$representation. Then the solution to eq. (3.8) can be written as

$$
a_{a}^{i}\left(x^{+}, \boldsymbol{x}_{\perp} ; k^{+}\right)=\frac{i}{2 k^{+}} \int \mathrm{d} y^{+} \mathrm{d} \boldsymbol{y}_{\perp} \mathcal{G}_{a b}\left(x^{+}, \boldsymbol{x}_{\perp} ; y^{+}, \boldsymbol{y}_{\perp} ; k^{+}\right) \mathcal{J}_{b}^{i}\left(y^{+}, \boldsymbol{y}_{\perp} ; k^{+}\right),
$$

where $\mathcal{J}^{i}$ refers to the total current in the r.h.s. of eq. (3.8) and the Green's function $\mathcal{G}$ obeys

$$
\left(i \mathcal{D}^{-}+\frac{\nabla_{\perp}^{2}}{2 k^{+}}\right) \mathcal{G}\left(x^{+}, \boldsymbol{x}_{\perp} ; y^{+}, \boldsymbol{y}_{\perp} ; k^{+}\right)=i \delta\left(x^{+}-y^{+}\right) \delta^{(2)}\left(\boldsymbol{x}_{\perp}-\boldsymbol{y}_{\perp}\right) .
$$

$\mathcal{G}$ is formally the same as the $D=2+1$ Schrödinger evolution operator for a quantummechanical particle with mass $k^{+}$and time $x^{+}$propagating in a time-dependent potential $g \mathcal{A}^{-}$. As well known, this propagator admits the following representation as a path integral:

$$
\mathcal{G}\left(x^{+}, \boldsymbol{x}_{\perp} ; y^{+}, \boldsymbol{y}_{\perp} ; k^{+}\right)=\int\left[D \boldsymbol{r}_{\perp}\left(z^{+}\right)\right] \exp \left\{i \frac{k^{+}}{2} \int_{y^{+}}^{x^{+}} \mathrm{d} z^{+} \dot{\boldsymbol{r}}_{\perp}^{2}\left(z^{+}\right)\right\} \mathcal{U}\left(x^{+}, y^{+} ;\left[\boldsymbol{r}_{\perp}\right]\right)
$$


with the paths $\boldsymbol{r}_{\perp}\left(z^{+}\right)$obeying the boundary conditions $\boldsymbol{r}_{\perp}\left(y^{+}\right)=\boldsymbol{y}_{\perp}$ and $\boldsymbol{r}_{\perp}\left(x^{+}\right)=\boldsymbol{x}_{\perp}$. The corresponding vacuum propagator $\left(\mathcal{A}^{-}=0\right)$ will be also needed:

$$
\mathcal{G}_{0}\left(x^{+}, \boldsymbol{x}_{\perp} ; y^{+}, \boldsymbol{y}_{\perp} ; k^{+}\right)=\Theta\left(x^{+}-y^{+}\right) \frac{k^{+}}{2 \pi i\left(x^{+}-y^{+}\right)} \exp \left\{i \frac{k^{+}\left(\boldsymbol{x}_{\perp}-\boldsymbol{y}_{\perp}\right)^{2}}{2\left(x^{+}-y^{+}\right)}\right\} .
$$

Note that eq. (3.11) goes beyond the eikonal approximation in the sense that the gluon trajectory is not a priori imposed (as we did for the quark and the antiquark), but rather is determined by the gluon interactions with the background field.

Given the solution $a^{i}$, the gluon emission amplitude is obtained as (for an on-shell gluon with 4-momentum $k^{\mu}$, color $a$, and polarization $\lambda$ )

$$
\mathcal{M}_{\lambda}^{a}\left(k^{+}, \boldsymbol{k}_{\perp}\right)=-\lim _{k^{2} \rightarrow 0} k^{2} a_{\mu}^{a}(k) \epsilon_{\lambda}^{\mu}(k), \quad \epsilon_{\lambda}^{\mu}\left(k^{+}, \boldsymbol{k}_{\perp}\right)=\left(0, \frac{\boldsymbol{\epsilon}_{\perp} \cdot \boldsymbol{k}_{\perp}}{k^{+}}, \boldsymbol{\epsilon}_{\perp}\right) .
$$

In the LC gauge $a^{+}=0$, this involves only the transverse components $a^{i}$, as anticipated. Using eq. (3.9) for $x^{+} \rightarrow \infty$ together with the following composition law (valid for $\left.x^{+}>z^{+}>y^{+}\right)$

$$
\mathcal{G}\left(x^{+}, \boldsymbol{x}_{\perp} ; y^{+}, \boldsymbol{y}_{\perp} ; k^{+}\right)=\int \mathrm{d} \boldsymbol{z}_{\perp} \mathcal{G}\left(x^{+}, \boldsymbol{x}_{\perp} ; z^{+}, \boldsymbol{z}_{\perp} ; k^{+}\right) \mathcal{G}\left(z^{+}, \boldsymbol{z}_{\perp} ; y^{+}, \boldsymbol{y}_{\perp} ; k^{+}\right),
$$

applied to $z^{+}=L^{+} \equiv \sqrt{2} L$ (notice that for $x^{+}>L^{+}$, one has $\mathcal{A}^{-}=0$ and then $\mathcal{G}=\mathcal{G}_{0}$ ), one obtains the amplitude as a sum of two pieces,

$$
\mathcal{M}_{a}^{i}\left(k^{+}, \boldsymbol{k}_{\perp}\right) \equiv-\lim _{k^{2} \rightarrow 0} k^{2} a_{a}^{i}(k)=\mathcal{M}_{a}^{i, \text { in }}+\mathcal{M}_{a}^{i, \text { out }}
$$

describing emissions inside the medium $\left(0<x^{+}<L^{+}\right)$and outside the medium $\left(x^{+}>L^{+}\right)$, respectively. Each of these pieces is a sum of quark and antiquark contributions and below we only show the respective quark contributions. The 'out' piece is the simplest:

$$
\begin{aligned}
\mathcal{M}_{a, q}^{i, \text { out }}\left(k^{+}, \boldsymbol{k}_{\perp}\right) & =\int \mathrm{d}^{4} x \mathrm{e}^{i k \cdot x} \Theta\left(x^{+}-L^{+}\right) \mathcal{J}_{a, q}^{i}(x) \\
& =g\left(u^{i}-v^{i}\right) \mathcal{U}_{q}^{a b}\left(L^{+}, 0\right) \mathcal{C}_{b} \int_{L^{+}}^{\infty} \mathrm{d} x^{+} \mathrm{e}^{i(k \cdot u) x^{+}}
\end{aligned}
$$

where $k^{-}=\boldsymbol{k}_{\perp}^{2} / 2 k^{+}, \mathcal{J}_{q}^{i}=J_{q}^{i}-\left(\partial^{i} / \partial^{+}\right) J_{q}^{+}$, and in the second line we have used the $\delta$-functions in eq. (3.5) to perform the integrations over $x^{-}$and $\boldsymbol{x}_{\perp}$ and denoted $v^{\mu} \equiv$ $k^{\mu} / k^{+}=\left(1, v^{-}, \boldsymbol{v}_{\perp}\right)$. The 'in' piece of the quark-emission amplitude reads

$$
\begin{aligned}
\mathcal{M}_{a, q}^{i, \text { in }}\left(k^{+}, \boldsymbol{k}_{\perp}\right)=g & \int_{0}^{L^{+}} \mathrm{d} x^{+} \mathrm{e}^{i k^{-} L^{+}+i\left(k^{+} u^{-}\right) x^{+}} \int \mathrm{d} \boldsymbol{z}_{\perp} \mathrm{e}^{-i \boldsymbol{k}_{\perp} \cdot \boldsymbol{z}_{\perp}} \\
& \left.\left(u^{i}+i \partial_{x}^{i} / k^{+}\right) \mathcal{G}_{a b}\left(L^{+}, \boldsymbol{z}_{\perp} ; x^{+}, \boldsymbol{x}_{\perp} ; k^{+}\right)\right|_{\boldsymbol{x}_{\perp}=\boldsymbol{u}_{\perp} x^{+}} \mathcal{U}_{q}^{b c}\left(x^{+}, 0\right) \mathcal{C}_{c} .
\end{aligned}
$$

The corresponding formulæ for the antiquark are obtained by replacing $u^{\mu} \rightarrow \bar{u}^{\mu}$ and $\mathcal{C}_{a} \rightarrow-\mathcal{C}_{a}$. 
Given the amplitude, the emission probability $\mathcal{P}$ and the gluon spectrum are obtained by taking the modulus squared and then summing over colors and polarizations:

$$
\omega \frac{\mathrm{d} N}{\mathrm{~d}^{3} \boldsymbol{k}}=\frac{1}{16 \pi^{3}} \mathcal{P}(\boldsymbol{k}), \quad \mathcal{P}(\boldsymbol{k}) \equiv \sum_{a, i}\left\langle\left|\mathcal{M}_{a}^{i}\right|^{2}\right\rangle,
$$

where we have written the gluon momentum in normal coordinates as $k^{\mu}=(\omega, \boldsymbol{k})$ with $\omega=|\boldsymbol{k}|$ and performed the polarization sum by using $\sum_{\lambda} \epsilon_{\lambda}^{i}(k) \epsilon_{\lambda}^{j *}(k)=\delta^{i j}$. The brackets in eq. (3.18) refer to the medium average according to eq. (3.3).

The amplitude $\mathcal{M}_{a}^{i}$ is truly a sum of four terms: $(q$, in), (q, out), $(\bar{q}$, in), and ( $\bar{q}$, out). Hence the emission probability in eq. (3.18) involves 16 terms: 8 of them describe direct emissions by either the quark or the antiquark, and 8 represent $q \bar{q}$ interference terms. Each of these types of contributions - direct or interference - involves three types of pieces: (in, in), (in, out), or (out, out). For the (in, in) contributions, the gluon is emitted inside the medium in both the direct amplitude and the complex conjugate one ${ }^{4}$ denoting the respective emission times as $x^{+}$and $y^{+}$, we have $0<x^{+}, y^{+}<L^{+}$. For the (in, out) terms, one has $0<x^{+}<L^{+}$and $y^{+}>L^{+}$, or vice-versa. Finally, for the (out, out) pieces, both $x^{+}$and $y^{+}$are larger than $L^{+}$.

We anticipate that, for the problem of medium-induced gluon radiation, the (in, in) contributions will be the most important ones, both for direct emissions and for the interference terms. Here, however, we shall start by computing the respective (out, out) pieces, with the purpose of illustrating the medium averaging and the phenomenon of color decoherence in the simplest possible setting. This will also allow us to make contact with the results in ref. [27].

\subsection{The 'out-out' terms as a warm up}

Consider first the direct gluon emission, say from the quark. Eq. (3.16) implies

$$
\begin{aligned}
\mathcal{P}_{q}^{\text {(out) }}(\boldsymbol{k}) & =g^{2}\left(\boldsymbol{u}_{\perp}-\boldsymbol{v}_{\perp}\right)^{2}\left\langle\mathcal{U}_{q}^{a b}\left(L^{+}, 0\right) \mathcal{C}_{b} \mathcal{C}_{d} \mathcal{U}_{q}^{\dagger a d}\left(L^{+}, 0\right)\right\rangle \int_{L^{+}}^{\infty} \mathrm{d} x^{+} \int_{L^{+}}^{\infty} \mathrm{d} y^{+} \mathrm{e}^{i(k \cdot u)\left(x^{+}-y^{+}\right)} \\
& =g^{2} C_{F} \frac{\left(\boldsymbol{u}_{\perp}-\boldsymbol{v}_{\perp}\right)^{2}}{(k \cdot u)^{2}}=\frac{2 g^{2} C_{F}}{\left(k^{+}\right)^{2}} \frac{1}{v \cdot u}
\end{aligned}
$$

where the second line follows after using $\mathcal{C}_{b} \mathcal{C}_{d}=\delta_{b d} C_{F} /\left(N_{c}^{2}-1\right)$. (This is the condition that, prior to the emission, the $q \bar{q}$ pair be in a color singlet state.) We have also used (recall that e.g. $v^{\mu}=\left(1, v^{-}, \boldsymbol{v}_{\perp}\right)$ with $v^{2}=0$ and hence $2 v^{-}=\boldsymbol{v}_{\perp}^{2}$ )

$$
\left(\boldsymbol{u}_{\perp}-\boldsymbol{v}_{\perp}\right)^{2}=\boldsymbol{u}_{\perp}^{2}+\boldsymbol{v}_{\perp}^{2}-2 \boldsymbol{u}_{\perp} \cdot \boldsymbol{v}_{\perp}=2\left(u^{-}+v^{-}-\boldsymbol{u}_{\perp} \cdot \boldsymbol{v}_{\perp}\right)=2 v \cdot u .
$$

An expression similar to eq. (3.21) but with $v \cdot u \rightarrow v \cdot \bar{u}$ holds for the direct emission by the antiquark. Note that there is no medium dependence in the final result for $\mathcal{P}_{q}^{\text {(out) }}$ because (a) the quark Wilson lines in the direct and the complex conjugate amplitude have compensated each other, and (b) there was a similar cancelation of the $L$-dependent

\footnotetext{
${ }^{4}$ It would be perhaps more appropriate to say that the gluon is emitted in the direct amplitude and reabsorbed in the complex conjugate amplitude. For brevity, we shall refer to all such processes as 'emissions'.
} 
phases $\mathrm{e}^{ \pm i(k \cdot u) L^{+}}$generated by the lower limit $L^{+}$of the time integrations. Accordingly, eq. (3.21) is formally identical to the corresponding probability in the vacuum, $\mathcal{P}_{q}^{(\mathrm{vac})}$. For later use, it is convenient to rewrite this vacuum probability as

$$
\mathcal{P}_{q}^{(\mathrm{vac})}(\boldsymbol{k})=2 g^{2} C_{F}\left(\boldsymbol{u}_{\perp}-\boldsymbol{v}_{\perp}\right)^{2} \tau_{q}^{2},
$$

where $\tau_{q}=1 /[\sqrt{2}(k \cdot u)]$ is the formation time for a gluon emission by the quark. Indeed,

$$
k \cdot u=\frac{\omega E_{q}}{p_{q}^{+}}\left(1-\cos \theta_{q}\right) \simeq \frac{\omega \theta_{q}^{2}}{2 \sqrt{2}} \quad \Longrightarrow \quad \tau_{q}=\frac{1}{\sqrt{2}(k \cdot u)} \simeq \frac{2}{\omega \theta_{q}^{2}},
$$

where one recognizes the expression (2.1) for the formation time at small angles. (We recall that the small angle approximations are strictly valid in the plasma rest frame.)

As explained in section 2, throughout this paper we are mostly interested in emissions at relatively large angles, for which the formation times are small: $\tau_{q} \ll L^{+}$. On the other hand, the (out, out) piece (3.19) of the emission probability is controlled, by construction, by emission times $x^{+}$and $y^{+}$within the range $L^{+} \leq x^{+}, y^{+} \lesssim L^{+}+\tau_{q}$, which are much larger than $\tau_{q}$. It would be very unnatural that vacuum-like emissions be delayed up to times much larger than the characteristic formation time $\tau_{q}$. But as a matter of facts, when $\tau_{q} \ll L^{+}$there is no physical out-of-medium emission; in that case, eq. (3.19) represents merely a piece of the total result which cancels against other pieces. More precisely, the boundary terms generated by emission times within an interval $\Delta x^{+} \simeq \tau_{q}$ around $L^{+}$ cancel when adding together the (in, in), (in, out), and (out, out) contributions. Such cancelations occur separately for the direct emissions and for the interference terms. The net result is that all the vacuum-like emissions at large angles are emitted at early times $x^{+}, y^{+} \lesssim \tau_{q} \ll L^{+}$, as expected on physical grounds.

The above argument also explains why the (out, out) piece plays no physical role for the situation of interest in this paper, which is characterized by small formation times: $\tau_{q}, \tau_{\bar{q}} \ll L$. On the other hand, this piece becomes important for the relatively soft and collinear emissions with relatively large formation times $\tau_{q}, \tau_{\bar{q}} \gtrsim L$. This is the situation considered in ref. [27]. To make contact with the results in that paper, we shall now consider the (out, out) contribution to the interference term. From eq. (3.16), this is obtained as

$$
\mathcal{I}^{\text {(out) }}(\boldsymbol{k})=-\frac{2 g^{2} C_{F}}{\left(k^{+}\right)^{2}} \frac{\left(u^{i}-v^{i}\right)\left(\bar{u}^{i}-v^{i}\right)}{(v \cdot u)(v \cdot \bar{u})} \cos \left[L^{+} k \cdot(u-\bar{u})\right] S_{q \bar{q}}\left(L^{+}, 0\right)
$$

where the quark and the antiquark Wilson lines combined in the following 2-point function

$$
S_{q \bar{q}}\left(L^{+}, 0\right)=\frac{1}{N_{c}^{2}-1}\left\langle\operatorname{Tr} \mathcal{U}_{q}\left(L^{+}, 0\right) \mathcal{U}_{\bar{q}}^{\dagger}\left(L^{+}, 0\right)\right\rangle
$$

which describes the residual color coherence between the two fermions after having crossed the medium - that is, the probability for the $q \bar{q}$ pair to remain in a color singlet state. $S_{q \bar{q}}$ is formally the same as the scattering $S$-matrix for a dipole made with a pair of colored particles in the adjoint representation that we shall succinctly refer to as the ' $q \bar{q}$ dipole'. 
For a background field with a Gaussian distribution, cf. eq. (3.3), the expectation value in eq. (3.24) is easily computed as (see e.g. [30])

$$
S_{\text {dip }}\left(x^{+}, y^{+} ;\left[\boldsymbol{r}_{\perp}\right]\right)=\exp \left\{-g^{2} N_{c} \int_{y^{+}}^{x^{+}} \mathrm{d} z^{+} n_{0}\left(z^{+}\right) \int \frac{\mathrm{d}^{2} \boldsymbol{q}_{\perp}}{(2 \pi)^{2}} \frac{1-\mathrm{e}^{i \boldsymbol{q}_{\perp} \cdot \boldsymbol{r}_{\perp}\left(z^{+}\right)}}{\left(\boldsymbol{q}_{\perp}^{2}+\mu_{D}^{2}\right)^{2}}\right\}
$$

where for later convenience we have kept generic endpoints in time, $y^{+}$and $x^{+}$, and a generic 'trajectory' $\boldsymbol{r}_{\perp}\left(z^{+}\right)$for the dipole transverse size in the interval $y^{+}<z^{+}<x^{+}$. For the cases of interest in this work, the dipole size is always much smaller than the medium screening length, $r \equiv\left|\boldsymbol{r}_{\perp}\right| \ll \mu_{D}^{-1}$. Then the integral over $\boldsymbol{q}_{\perp}$ in eq. (3.25) is controlled by transverse momenta within the range $\mu_{D}<q_{\perp}<1 / r$ and to leading logarithmic accuracy can be estimated by expanding $\mathrm{e}^{i \boldsymbol{q}_{\perp} \cdot \boldsymbol{r}_{\perp}}$ to second order. This yields

$$
S_{\text {dip }}\left(x^{+}, y^{+} ;\left[\boldsymbol{r}_{\perp}\right]\right) \simeq \exp \left\{-\frac{\alpha_{s} N_{c}}{4} \int_{y^{+}}^{x^{+}} \mathrm{d} z^{+} n_{0}\left(z^{+}\right) r^{2}\left(z^{+}\right) \ln \frac{1}{r^{2}\left(z^{+}\right) \mu_{D}^{2}}\right\},
$$

where the $\operatorname{logarithm} \rho \equiv \ln \left(1 / r^{2} \mu_{D}^{2}\right)$ is assumed to be relatively large, $\rho \gg 1$. A more compact version of eq. (3.26) can be obtained by assuming $n_{0}\left(z^{+}\right)=n_{0}$ to be constant and neglecting the variation of the logarithm within the interval of integration; then,

$$
S_{\text {dip }}\left(x^{+}, y^{+} ;\left[\boldsymbol{r}_{\perp}\right]\right) \simeq \exp \left\{-\frac{1}{4} \hat{q} \rho \int_{y^{+}}^{x^{+}} \mathrm{d} z^{+} r^{2}\left(z^{+}\right)\right\},
$$

where $^{5}$ (the saturation scale $Q_{s}$ is introduced for later reference)

$$
\hat{q} \equiv \alpha_{s} N_{c} n_{0} \sim \alpha_{s} N_{c} \mu_{D}^{2} T, \quad Q_{s}^{2} \equiv \hat{q} L^{+},
$$

and it is understood that the $\operatorname{logarithm} \rho$ in eq. (3.27) is evaluated with the maximal dipole size $r_{\max }$ within the interval $y^{+}<z^{+}<x^{+}$.

When applying these formulæ to the $q \bar{q}$ dipole in eq. (3.24), one has $\boldsymbol{r}_{\perp}\left(z^{+}\right)=\left(\boldsymbol{u}_{\perp}-\right.$ $\left.\overline{\boldsymbol{u}}_{\perp}\right) z^{+}$with $0<z^{+}<L^{+}$and therefore

$$
S_{q \bar{q}}\left(L^{+}, 0\right) \simeq \exp \left\{-\frac{1}{12} \hat{q} \rho\left(\boldsymbol{u}_{\perp}-\overline{\boldsymbol{u}}_{\perp}\right)^{2}\left(L^{+}\right)^{3}\right\} \simeq \exp \left\{-\frac{1}{24}\left(Q_{s} \theta_{q \bar{q}} L^{+}\right)^{2} \rho\right\},
$$

where in writing the second estimate we have used a small-angle approximation which holds, strictly speaking, in the plasma rest frame (the product $\theta_{q \bar{q}} L^{+}$is boost invariant):

$$
\left(\boldsymbol{u}_{\perp}-\overline{\boldsymbol{u}}_{\perp}\right)^{2}=2 u \cdot \bar{u}=2 \frac{p_{q} \cdot p_{\bar{q}}}{p_{q}^{+} p_{\bar{q}}^{+}}=2 \frac{E_{q} E_{\bar{q}}}{p_{q}^{+} p_{\bar{q}}^{+}}\left(1-\cos \theta_{q \bar{q}}\right) \simeq \frac{\theta_{q \bar{q}}^{2}}{2} .
$$

At this point one should recall that we consider a relatively large dipole angle, $\theta_{q \bar{q}} \gg \theta_{c}$ or $Q_{s} \theta_{q \bar{q}} L^{+} \gg 1$. The exponent in eq. (3.29) is therefore large, which implies that $S_{q \bar{q}} \ll 1$ : the (out, out) contribution to interference is washed out by color decoherence in the

\footnotetext{
${ }^{5}$ Eqs. (3.28) and (2.3) are consistent with each other since $\ell \sim 1 /\left(\alpha_{s} N_{c} T\right)$ for a weakly-coupled QGP.
} 
medium. Since they follow different trajectories, the quark and the antiquark undergo different color precessions, so after leaving the medium they do not form a color singlet anymore. We see that, in the soft and collinear regime where the (out, out) piece yields a physical contribution, the corresponding interference term vanishes. Then the total outof-medium radiation by the dipole is simply the incoherent sum of two vacuum-like contributions (cf. eq. (3.19)), by the quark and the antiquark. This is the main conclusion in ref. [27] and admits interesting consequences: it implies that, in the presence of the medium, there should be an enhancement in the radiation at large angles, outside the dipole cone: $\theta_{q}, \theta_{\bar{q}}>\theta_{q \bar{q}}$.

To fully appreciate this conclusion, it is important to specify the kinematical region where it applies. From the previous arguments, it is clear that this relies on two main assumptions: (i) relatively large formation times ${ }^{6} \tau_{q}, \tau_{\bar{q}} \gtrsim L$, and (ii) a sufficiently large dipole angle $\theta_{q \bar{q}} \gg \theta_{c}$. As we shall now argue, these conditions are satisfied for sufficiently soft gluons and for relatively small, but not too small, emission $\left(\theta_{q}, \theta_{\bar{q}}\right)$ and dipole $\left(\theta_{q \bar{q}}\right)$ angles. The precise conditions read

$$
\omega \ll \omega_{c} \quad \text { and } \quad \theta_{c} \ll \theta_{q}, \theta_{\bar{q}}, \theta_{q \bar{q}} \lesssim \theta_{c}\left(\frac{\omega_{c}}{\omega}\right)^{1 / 2} .
$$

The upper limit on $\omega$ comes up by combining the two conditions above and focusing on emission angles which are commensurable with the dipole angle: $\theta_{q} \sim \theta_{\bar{q}} \sim \theta_{q \bar{q}}$ (this is the regime where the conclusion in ref. [27] have non-trivial consequences). Then, one can write

$$
\tau_{q} \simeq \frac{2}{\omega \theta_{q}^{2}} \gtrsim L \quad \Longrightarrow \quad \omega \lesssim \frac{2}{L \theta_{q \bar{q}}^{2}} \ll \frac{2}{L \theta_{c}^{2}}=\omega_{c},
$$

where we have used $\theta_{q} \sim \theta_{q \bar{q}} \gg \theta_{c}$ and $\theta_{c}^{2}=2 /\left(\omega_{c} L\right)$, cf. eq. (2.5). Then the upper limit on the values of the angles follows by rewriting the condition $\tau_{q} \gtrsim L$ in the form

$$
\theta_{q} \lesssim \sqrt{\frac{2}{\omega L}}=\theta_{c} \sqrt{\frac{\omega_{c}}{\omega}}
$$

It should be also clear from the above that for a very small dipole angle $\theta_{q \bar{q}} \lesssim \theta_{c}$, the medium effects become irrelevant (since the $q \bar{q}$ pair preserves its color coherence throughout the medium), so the soft emissions with $\tau_{q} \gtrsim L$ proceed exactly as in the vacuum - in particular, the dipole antenna shows the characteristic angular ordering.

Note that the region (3.31) has some overlap with the 'small-angle regime' for mediuminduced gluon radiation as defined in section 2.2 - that is, the regime characterized by $\theta_{c} \ll \theta_{q \bar{q}} \ll \theta_{f}$. There is, however, an important difference: the respective range in section 2.2 refers to the dipole angle $\theta_{q \bar{q}}$ alone; while this angle can be as small as $\theta_{c}$, the actual emission angles for the BDMPS-Z gluons are much larger: $\theta_{q}, \theta_{\bar{q}} \gtrsim \theta_{f} \gg \theta_{c}$. On the other hand, the upper limit in eq. (3.31) is much smaller than $\theta_{f}$, as it can be easily

\footnotetext{
${ }^{6}$ Formally, this condition is necessary to avoid the rapid oscillations of the cosine factor in eq. (3.23), whose argument is the same as $L^{+} k \cdot(u-\bar{u})=L / \tau_{q}-L / \tau_{\bar{q}}$. Less formally, the regime of large formation times $\gtrsim L$ is the only one where the (out, out) piece of the spectrum is physically irrelevant, as already explained.
} 
checked using eq. (2.10). So, even for dipole angles $\theta_{q \bar{q}}$ as small as shown in eq. (3.31), the out-of-medium emissions discussed in ref. [27] and the BDMPS-Z-like emissions that we presently focus on are geometrically separated, with the latter being distributed at significantly larger angles than the former.

\section{Medium-induced gluon radiation: direct emission}

Starting with this section, we shall concentrate on the in-medium, or (in, in), pieces, which are the dominant contributions to medium-induced gluon radiation in the kinematical range of interest (relatively small frequencies, $\omega \ll \omega_{c}$, or large emission angles $\theta_{q} \gtrsim \theta_{f}(\omega) \gg$ $\left.\theta_{c}\right)$. Although we are ultimately interested in the quark-antiquark interference terms, for which we shall present original results in the next section, we shall start our analysis with the direct emission terms, from which we shall extract the BDMPS-Z spectrum. This will give us the opportunity to develop a series of approximations that we shall test on the case of direct emissions and then apply to the interference terms. More precise calculations, which confirm the results to be obtained in sections 4 and 5 , will be presented in appendix A.

The probability for in-medium gluon radiation by the quark is obtained by taking the modulus squared of the amplitude (3.17), summing over the final color indices, averaging over the initial ones, and performing the medium average over the background field. This yields

$$
\begin{aligned}
\mathcal{P}_{q}^{(\mathrm{in})}(\boldsymbol{k})= & 2 g^{2} C_{F} \operatorname{Re} \int_{0}^{L^{+}} \mathrm{d} x^{+} \int_{0}^{x^{+}} \mathrm{d} y^{+} \mathrm{e}^{i k^{+} u^{-}\left(x^{+}-y^{+}\right)} \\
& \times \int \mathrm{d} \boldsymbol{z}_{1 \perp} \int \mathrm{d} \boldsymbol{z}_{2 \perp} \mathrm{e}^{-i \boldsymbol{k}_{\perp} \cdot\left(\boldsymbol{z}_{1 \perp}-\boldsymbol{z}_{2 \perp}\right)}\left(u^{i}+i \partial_{x}^{i} / k^{+}\right)\left(u^{i}-i \partial_{y}^{i} / k^{+}\right) \\
& \times \frac{1}{N_{c}^{2}-1}\left\langle\operatorname{Tr} \mathcal{G}\left(L^{+}, \boldsymbol{z}_{1 \perp} ; x^{+}, \boldsymbol{x}_{\perp} ; k^{+}\right) \mathcal{U}_{q}\left(x^{+}, y^{+}\right) \mathcal{G}^{\dagger}\left(L^{+}, \boldsymbol{z}_{2 \perp} ; y^{+}, \boldsymbol{y}_{\perp} ; k^{+}\right)\right\rangle,
\end{aligned}
$$

where $\mathcal{U}_{q}\left(x^{+}, y^{+}\right)$is given by eq. (3.7) with $\boldsymbol{r}_{\perp}\left(z^{+}\right)=\boldsymbol{u}_{\perp} z^{+}$and it is understood that after the performing the transverse derivatives $\partial_{x}^{i}$ and $\partial_{y}^{i}$ one sets $\boldsymbol{x}_{\perp}=\boldsymbol{u}_{\perp} x^{+}$and $\boldsymbol{y}_{\perp}=\boldsymbol{u}_{\perp} y^{+}$. In writing eq. (4.1) we have restricted the time integrals to $0<y^{+}<x^{+}<L^{+}$and multiplied the result by a factor of 2 . The Feynman graph representing this emission is shown in figure 4.

Note that the quark Wilson lines prior to the first emission time $y^{+}$have canceled each other between the direct and the complex conjugate amplitude. The color trace in the last line of eq. (4.1) can be further simplified by using the fact that the background field correlations are local in time. To that aim one first uses the composition law (3.14) to break the last gluon propagator in eq. (4.1) into two pieces - from $y^{+}$to $x^{+}$and from $x^{+}$to $L^{+}$. Then the medium average factorizes as (below, the $k^{+}$variable is kept implicit, 


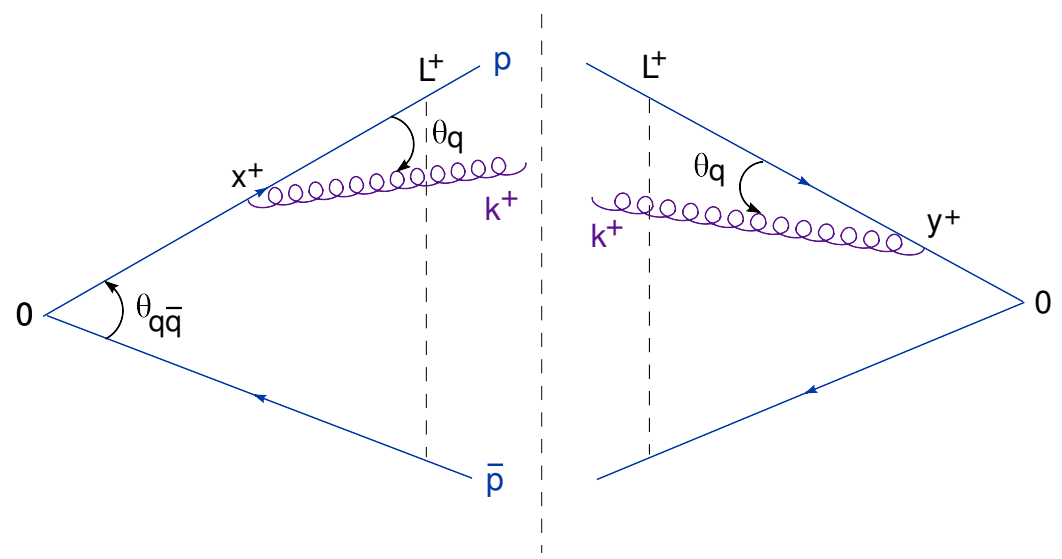

Figure 4. The standard representation of the Feynman graph for direct emission by the quark (amplitude times the complex conjugate amplitude).

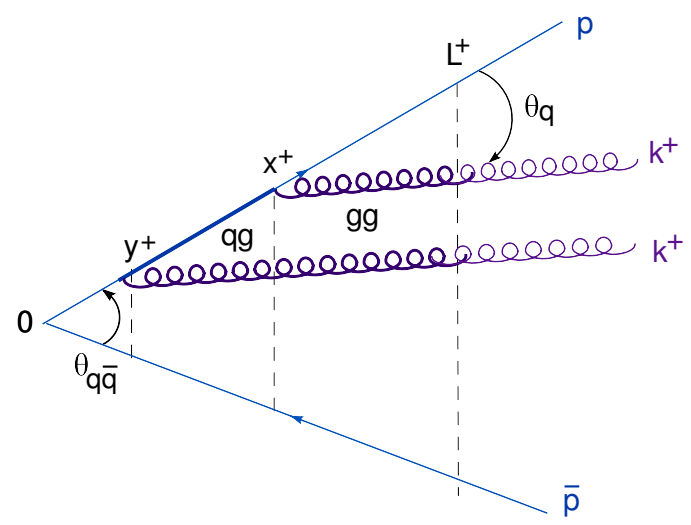

Figure 5. A folded version of the Feynman graph for direct emission where the amplitude and the complex conjugate amplitude are represented on top of each other, to more clearly exhibit the $q g$ and $g g$ dipoles. The (quark and gluon) Wilson lines are indicated with thick lines.

to simplify writing)

$$
\begin{aligned}
\int \mathrm{d} \boldsymbol{z}_{\perp} \frac{1}{N_{c}^{2}-1}\langle & \left.\operatorname{Tr} \mathcal{G}\left(L^{+}, \boldsymbol{z}_{1 \perp} ; x^{+}, \boldsymbol{x}_{\perp}\right) \mathcal{U}_{q}\left(x^{+}, y^{+}\right) \mathcal{G}^{\dagger}\left(x^{+}, \boldsymbol{z}_{\perp} ; y^{+}, \boldsymbol{y}_{\perp}\right) \mathcal{G}^{\dagger}\left(L^{+}, \boldsymbol{z}_{2 \perp} ; x^{+}, \boldsymbol{z}_{\perp}\right)\right\rangle \\
= & \int \mathrm{d} \boldsymbol{z}_{\perp} \frac{1}{N_{c}^{2}-1}\left\langle\operatorname{Tr} \mathcal{G}\left(L^{+}, \boldsymbol{z}_{1 \perp} ; x^{+}, \boldsymbol{x}_{\perp}\right) \mathcal{G}^{\dagger}\left(L^{+}, \boldsymbol{z}_{2 \perp} ; x^{+}, \boldsymbol{z}_{\perp}\right)\right\rangle \\
& \times \frac{1}{N_{c}^{2}-1}\left\langle\operatorname{Tr} \mathcal{U}_{q}\left(x^{+}, y^{+}\right) \mathcal{G}^{\dagger}\left(x^{+}, \boldsymbol{z}_{\perp} ; y^{+}, \boldsymbol{y}_{\perp}\right)\right\rangle
\end{aligned}
$$

The two color traces in the r.h.s. of eq. (4.2) are recognized as the 2-body propagators of two effective dipoles - a quark-gluon $(q g)$ dipole extending from $y^{+}$to $x^{+}$and a gluongluon $(g g)$ dipole from $x^{+}$to $L^{+}$- whose interactions in the medium are here computed beyond the eikonal approximation (cf. the discussion after eq. (3.12)). These dipoles can be easier visualized by folding the Feynman graph in figure 4 in such a way that the direct and complex conjugate amplitudes overlap with each other, as shown in figure 5. 
Using (3.11), one obtains the following path-integral representation for the propagator of the $q g$ dipole:

$$
\begin{aligned}
\mathcal{K}_{q g}\left(x^{+}, \boldsymbol{z}_{\perp} ; y^{+}, \boldsymbol{y}_{\perp} ; k^{+}\right) & \equiv \frac{1}{N_{c}^{2}-1}\left\langle\operatorname{Tr} \mathcal{U}_{q}\left(x^{+}, y^{+}\right) \mathcal{G}^{\dagger}\left(x^{+}, \boldsymbol{z}_{\perp} ; y^{+}, \boldsymbol{y}_{\perp}\right)\right\rangle \\
& =\int\left[D \boldsymbol{r}_{\perp}\right] \exp \left\{-i \frac{k^{+}}{2} \int_{y^{+}}^{x^{+}} \mathrm{d} z^{+} \dot{\boldsymbol{r}}_{\perp}^{2}\right\} S_{q g}\left(x^{+}, y^{+} ;\left[\boldsymbol{r}_{\perp}-\boldsymbol{u}_{\perp} z^{+}\right]\right),
\end{aligned}
$$

which features a $q g$ pair with fluctuating size $\boldsymbol{r}_{\perp}\left(z^{+}\right)-\boldsymbol{u}_{\perp} z^{+}$and path-dependent $S$-matrix

$$
S_{q g}\left(x^{+}, y^{+} ;\left[\boldsymbol{r}_{\perp}-\boldsymbol{u}_{\perp} z^{+}\right]\right) \simeq \exp \left\{-\frac{1}{4} \hat{q} \rho \int_{y^{+}}^{x^{+}} \mathrm{d} z^{+}\left(\boldsymbol{r}_{\perp}\left(z^{+}\right)-\boldsymbol{u}_{\perp} z^{+}\right)^{2}\right\} .
$$

We recall that the boundary conditions for the gluon paths are $\boldsymbol{r}_{\perp}\left(y^{+}\right)=\boldsymbol{y}_{\perp}$ and $\boldsymbol{r}_{\perp}\left(x^{+}\right)=$ $\boldsymbol{z}_{\perp}$ and that $\rho$ is a slowly varying function of the dipole size (cf. eq. (3.26)).

As for the $g g$ dipole in eq. (4.2), the corresponding mathematics turns out to be simpler: on the average, the medium is homogeneous in the transverse plane, as manifest on eq. (3.3). Then the medium averaging also averages out the fluctuations in the dipole transverse size, with the net effect that the respective $S$-matrix depends only upon the initial dipole size at time $x^{+}$, that is $\boldsymbol{x}_{\perp}-\boldsymbol{z}_{\perp}$. Specifically, the following identity holds (see e.g. $[19,33]$ for details):

$$
\begin{gathered}
\int \mathrm{d} \boldsymbol{z}_{1 \perp} \int \mathrm{d} \boldsymbol{z}_{2 \perp} \mathrm{e}^{-i \boldsymbol{k}_{\perp} \cdot\left(\boldsymbol{z}_{1 \perp}-\boldsymbol{z}_{2 \perp}\right)} \frac{1}{N_{c}^{2}-1}\left\langle\operatorname{Tr} \mathcal{G}\left(L^{+}, \boldsymbol{z}_{1 \perp} ; x^{+}, \boldsymbol{x}_{\perp}\right) \mathcal{G}^{\dagger}\left(L^{+}, \boldsymbol{z}_{2 \perp} ; x^{+}, \boldsymbol{z}_{\perp}\right)\right\rangle \\
=\mathrm{e}^{-i \boldsymbol{k}_{\perp} \cdot\left(\boldsymbol{x}_{\perp}-\boldsymbol{z}_{\perp}\right)} S_{g g}\left(L^{+}, x^{+} ; \boldsymbol{x}_{\perp}-\boldsymbol{z}_{\perp}\right),
\end{gathered}
$$

with (compare to eq. (3.27))

$$
S_{g g}\left(L^{+}, x^{+} ; \boldsymbol{x}_{\perp}-\boldsymbol{z}_{\perp}\right) \simeq \exp \left\{-\frac{1}{4} \hat{q} \rho\left(L^{+}-x^{+}\right)\left(\boldsymbol{x}_{\perp}-\boldsymbol{z}_{\perp}\right)^{2}\right\} .
$$

Note that in writing eqs. (4.4) and (4.6) above, we have tacitly assumed that the respective dipole sizes are much smaller than $\mu_{D}^{-1}$, so that the approximations leading to eqs. (3.26)(3.27) indeed apply. This will be checked later, when we shall see that the typical dipole sizes are of order $1 / k_{f}$ for (4.4) and respectively of order $1 / Q_{s}$ for (4.6).

Putting together the previous results, we deduce the following expression for the probability for direct emission from the quark

$$
\begin{aligned}
\mathcal{P}_{q}^{(\mathrm{in})}(\boldsymbol{k})= & 2 g^{2} C_{F} \operatorname{Re} \int_{0}^{L^{+}} \mathrm{d} x^{+} \int_{0}^{x^{+}} \mathrm{d} y^{+} \mathrm{e}^{i k^{+} u^{-}\left(x^{+}-y^{+}\right)}\left(u^{i}+i \partial_{x}^{i} / k^{+}\right)\left(u^{i}-i \partial_{y}^{i} / k^{+}\right) \\
& \times \int \mathrm{d} \boldsymbol{z}_{\perp} \mathrm{e}^{-i \boldsymbol{k}_{\perp} \cdot\left(\boldsymbol{x}_{\perp}-\boldsymbol{z}_{\perp}\right)} \mathcal{K}_{q g}\left(x^{+}, \boldsymbol{z}_{\perp} ; y^{+}, \boldsymbol{y}_{\perp} ; k^{+}\right) S_{g g}\left(L^{+}, x^{+} ; \boldsymbol{x}_{\perp}-\boldsymbol{z}_{\perp}\right),
\end{aligned}
$$

where it is understood that $\boldsymbol{x}_{\perp} \rightarrow \boldsymbol{u}_{\perp} x^{+}$and $\boldsymbol{y}_{\perp} \rightarrow \boldsymbol{u}_{\perp} y^{+}$after taking the derivatives. Within the limits of our calculation, this expression is exact. It is also rather formal, in the sense of involving a path integral and holding for an arbitrary kinematics of the emitted gluon. In the 'harmonic approximation', which consists in treating the slowly varying 
logarithm $\rho$ in eqs. (4.4) and (4.6) as a fixed quantity, the integrations become Gaussian and can be performed exactly (see the appendix). To keep the discussion as intuitive as possible, in what follows we shall perform a series of approximations which are valid in the kinematics of interest.

But before we proceed with more formal steps, let us emphasize a point of physics: ${ }^{7}$ the gluon formation time $\tau_{f}$ for medium-induced radiation is controlled by the intermediate, quark-gluon dipole, stage of the dynamics in eq. (4.7) and hence it is of the order of the typical duration $x^{+}-y^{+}$of that stage. Indeed, the $S$-matrix (4.4) of this effective dipole, built with the quark in the direct amplitude and the gluon in the complex conjugate one (or vice-versa), is a measure of the color coherence between the emitted gluon and the parent quark. So long as this dipole is relatively small (meaning for sufficiently small time separations $x^{+}-y^{+}$), one has $S_{q g} \simeq 1$ and then one cannot distinguish the gluon from the quark: in any process involving color exchanges, the emerging quark-gluon pair acts in the same way as the original, bare, quark would do. But with increasing $x^{+}-y^{+}$, the dipole size increases (via gluon diffusion) and then $S_{q g}$ starts to decrease from one, because of the medium rescattering. One can consider the gluon as being formed when the $q g$ dipole suffers a first inelastic collision in the medium, i.e. when the exponent in $S_{q g}$ becomes of $\mathcal{O}(1)$. The respective value of $x^{+}-y^{+}$sets the formation time. For even larger values of $x^{+}$, one has $S_{q g} \ll 1$ and the emission probability is strongly suppressed.

The starting point of our approximation is an expression for the propagator (4.3) of the $q g$ dipole valid in the harmonic approximation. With $\rho \approx$ const. and absorbed into the normalization of $\hat{q}$ for convenience, ${ }^{8}$ the path integral (4.3) describes a harmonic oscillator with imaginary squared frequency

$$
\Omega^{2}=i \frac{\hat{q}}{2 k^{+}} \quad \Longrightarrow \quad \Omega=\frac{1+i}{\sqrt{2}} \sqrt{\frac{\hat{q}}{2 k^{+}}},
$$

and hence it can be exactly computed (see e.g. $[11,19]$ for details and also the appendix below). To be specific, let us ignore the transverse derivatives in eq. (4.7) for the time being (we shall return to them latter) and fix $\boldsymbol{x}_{\perp}=\boldsymbol{u}_{\perp} x^{+}$and $\boldsymbol{y}_{\perp}=\boldsymbol{u}_{\perp} y^{+}$. Then one obtains

$$
\begin{aligned}
\mathcal{K}_{q g}\left(x^{+}, \boldsymbol{b}_{\perp}+\boldsymbol{u}_{\perp} x^{+} ; y^{+}, \boldsymbol{u}_{\perp} y^{+} ; k^{+}\right)= & \exp \left\{-i k^{+}\left[\left(x^{+}-y^{+}\right) \frac{\boldsymbol{u}_{\perp}^{2}}{2}+\boldsymbol{u}_{\perp} \cdot \boldsymbol{b}_{\perp}\right]\right\} \\
& \times \mathcal{K}_{q g}\left(x^{+}, \boldsymbol{b}_{\perp} ; y^{+}, \mathbf{0}_{\perp} ; k^{+}\right)
\end{aligned}
$$

where we set $\boldsymbol{b}_{\perp} \equiv \boldsymbol{z}_{\perp}-\boldsymbol{u}_{\perp} x^{+}$and

$\mathcal{K}_{q g}\left(x^{+}, \boldsymbol{b}_{\perp} ; y^{+}, \mathbf{0}_{\perp} ; k^{+}\right)=\frac{k^{+} \Omega}{2 \pi i \sinh \Omega\left(x^{+}-y^{+}\right)} \exp \left\{-\frac{k^{+} \Omega}{2 i} \operatorname{coth} \Omega\left(x^{+}-y^{+}\right) \boldsymbol{b}_{\perp}^{2}\right\}$

\footnotetext{
${ }^{7}$ We would like to thank Al Mueller for an illuminating discussion of this point.

${ }^{8}$ This is a standard convention in the literature; the factors of $\rho$ can be recovered, if needed, by replacing everywhere $\hat{q} \rightarrow \hat{q} \rho$.
} 
The quantity $\boldsymbol{b}_{\perp}$ is the transverse size of the $q g$ dipole at time $x^{+}$and thus also the size of the ensuing $g g$ dipole at any time $z^{+}>x^{+}$. We shall denote

$$
\tau_{f} \equiv \frac{1}{|\Omega|}=\sqrt{\frac{2 k^{+}}{\hat{q}}},
$$

anticipating that this quantity plays the role of the formation time.

Two limits of eq. (4.10) will be useful in what follows:

(i) Small times $|\Omega|\left(x^{+}-y^{+}\right) \ll 1$ or $x^{+}-y^{+} \ll \tau_{f}$ : then, by expanding the r.h.s. of eq. (4.10) to quadratic order in $|\Omega|\left(x^{+}-y^{+}\right)$one finds

$$
\mathcal{K}_{q g}\left(x^{+}, \boldsymbol{b}_{\perp} ; y^{+}, \mathbf{0}_{\perp} ; k^{+}\right) \simeq \frac{k^{+}}{2 \pi i\left(x^{+}-y^{+}\right)} \exp \left\{-i \frac{k^{+} b_{\perp}^{2}}{2\left(x^{+}-y^{+}\right)}-\frac{1}{12} \hat{q}\left(x^{+}-y^{+}\right) b_{\perp}^{2}\right\} .
$$

This is recognized as the saddle point approximation to (4.3) with the saddle point determined by the kinetic piece of the action alone; that is, $\mathcal{K}_{q g} \approx \mathcal{G}_{0} S_{q g}$ where $\mathcal{G}_{0}$ is the free propagator (3.12) and $S_{q g}$ is the $S$-matrix (4.4) evaluated along the classical path, which reads:

$$
\boldsymbol{r}_{\text {class }}\left(z^{+}\right)-\boldsymbol{u}_{\perp} z^{+}=\frac{z^{+}-y^{+}}{x^{+}-y^{+}} \boldsymbol{b}_{\perp} .
$$

(ii) Large times $|\Omega|\left(x^{+}-y^{+}\right) \gg 1$ or $x^{+}-y^{+} \gg \tau_{f}$ : then, one finds

$$
\mathcal{K}_{q g}\left(x^{+}, \boldsymbol{b}_{\perp} ; y^{+}, \mathbf{0}_{\perp} ; k^{+}\right) \propto \frac{k^{+}}{\tau_{f}} \mathrm{e}^{-\left(x^{+}-y^{+}\right) / \tau_{f}} \exp \left\{-\frac{1+i}{4} \sqrt{\hat{q} k^{+}} b_{\perp}^{2}\right\} .
$$

Eq. (4.12) shows that at early times $x^{+}-y^{+} \lesssim \tau_{f}$ the size of the $q g$ dipole increases through diffusion, $b_{\perp}^{2} \propto\left(x^{+}-y^{+}\right) / k^{+}$(cf. the first term in the exponent) and this increase enhances the dipole rescattering off the medium (the second term in the exponent, as coming from $S_{q g}$ ). When $x^{+}-y^{+} \simeq \tau_{f}$, this second term becomes of order one, showing that $\tau_{f}$ is the formation time, as anticipated. For larger times $x^{+}-y^{+} \gg \tau_{f}$, the dipole propagator is exponentially suppressed, cf. eq. (4.14), meaning that the color coherence of the $q g$ pair has been destroyed by the medium. The maximal size of the $q g$ dipole, as attained for $x^{+}-y^{+} \simeq \tau_{f}$, is ${ }^{9}$

$$
b_{f}^{2} \simeq \frac{\tau_{f}}{k^{+}} \sim \frac{1}{\sqrt{\hat{q} \omega}} .
$$

Via the uncertainty principle, this yields the typical transverse momentum of the gluon at the formation time as $k_{f}^{2} \simeq 1 / b_{f}^{2} \simeq \sqrt{\omega \hat{q}}$, in agreement with eq. (2.4). This obeys the scaling law $k_{f}^{2} \simeq \hat{q} \tau_{f}$ showing that this transverse momentum has been acquired via medium rescattering during a time $\tau_{f}$. Since $\tau_{f} \ll L^{+}$, this $k_{\perp}$ is much smaller than the final momentum of the gluon, which can be as large as $k_{\perp} \sim Q_{s}$, as we shall shortly see.

To compute the final gluon spectrum, one has to also take into account the medium rescattering after the time of formation, as encoded in the $S$-matrix (4.6) of the $g g$ dipole.

\footnotetext{
${ }^{9}$ As usual, when writing parametric estimates, we ignore numerical factors and identify $k^{+}$with $\omega$.
} 
Specifically, the function $S_{g g}\left(L^{+}, x^{+} ; \boldsymbol{x}_{\perp}-\boldsymbol{z}_{\perp}\right)$ controls the range of the integration over $\boldsymbol{z}_{\perp}=\boldsymbol{b}_{\perp}+\boldsymbol{u}_{\perp} x^{+}$, which in turn fixes the final transverse momentum $\boldsymbol{k}_{\perp}$ via the Fourier transform in eq. (4.7). As we shall shortly check, the typical values for $b_{\perp}$ allowed by the $g g$ dipole are much smaller than $b_{f}$. Hence, in evaluating this Fourier transform, one can replace the propagator $\mathcal{K}_{q g}$ of the $q g$ dipole by the corresponding free propagator $\mathcal{G}_{0}$ (which carries the whole dependence upon $\boldsymbol{b}_{\perp}$ in the limit where $b_{\perp} \ll b_{f}$ ). Then the integral over $\boldsymbol{b}_{\perp}$ reduces to

$$
\mathrm{e}^{-i \frac{k^{+} \boldsymbol{u}_{\perp}^{2}}{2}\left(x^{+}-y^{+}\right)} \int \mathrm{d} \boldsymbol{b}_{\perp} \mathrm{e}^{i \boldsymbol{b}_{\perp} \cdot\left(\boldsymbol{k}_{\perp}-k^{+} \boldsymbol{u}_{\perp}\right)} \exp \left\{\frac{-i k^{+} b_{\perp}^{2}}{2\left(x^{+}-y^{+}\right)}\right\} \exp \left\{-\frac{\hat{q}}{4}\left(L^{+}-x^{+}\right) b_{\perp}^{2}\right\}
$$

where it was important to also include the phase factor in the r.h.s. of eq. (4.9) (which is a part of the free propagator $\left.\mathcal{G}_{0}\left(x^{+}, \boldsymbol{b}_{\perp}+\boldsymbol{u}_{\perp} x^{+} ; y^{+}, \boldsymbol{u}_{\perp} y^{+} ; k^{+}\right)\right)$. The overall phase in front of the above integral is such that it exactly cancels the phase $\mathrm{e}^{i k^{+} u^{-}\left(x^{+}-y^{+}\right)}$in eq. (4.7). This is worth emphasizing in view of the discussion of the interference terms in section 5 , where the corresponding phase cancelation does not hold — which in turn has important consequences.

For medium-induced radiation, the time variables $x^{+}$and $y^{+}$can lie anywhere within the medium, $0<x^{+}, y^{+}<L^{+}$(except very close to the boundaries ${ }^{10}$ ), with the condition that $x^{+}-y^{+} \sim \tau_{f} \ll L^{+}$. Accordingly $\hat{q}\left(L^{+}-x^{+}\right)$is parametrically of the same order as $\hat{q} L^{+}=Q_{s}^{2}$, and thus is much larger than $k^{+} / \tau_{f} \sim k_{f}^{2}$. Hence the integral in eq. (4.16) is controlled by the last factor inside the integrand and yields

$$
\int \mathrm{d} \boldsymbol{b}_{\perp} \mathrm{e}^{i \boldsymbol{b}_{\perp} \cdot\left(\boldsymbol{k}_{\perp}-k^{+} \boldsymbol{u}_{\perp}\right)} \exp \left\{-\frac{1}{4} Q_{s}^{2} b_{\perp}^{2}\right\} \sim \frac{1}{Q_{s}^{2}} \exp \left\{-\frac{\left(\boldsymbol{k}_{\perp}-k^{+} \boldsymbol{u}_{\perp}\right)^{2}}{Q_{s}^{2}}\right\} .
$$

Note that $k^{+} \boldsymbol{u}_{\perp}$ is the transverse momentum inherited by the gluon from its parent quark. Accordingly, $\boldsymbol{k}_{\perp}-k^{+} \boldsymbol{u}_{\perp}$ is the additional transverse momentum acquired by the gluon from the medium and is the same as the component of the gluon momentum which is transverse to the quark; indeed, using eq. (3.22) one can write

$$
\left(\boldsymbol{k}_{\perp}-k^{+} \boldsymbol{u}_{\perp}\right)^{2}=2 k^{+}(k \cdot u) \simeq\left(\omega \theta_{q}\right)^{2} .
$$

Hence, eq. (4.17) shows that the momentum gained by the gluon via medium rescattering can be as large as $Q_{s}$, as anticipated. Since $Q_{s} \gg k_{f}$, it is clear that most of this momentum gets accumulated after the gluon formation. This is confirmed by the fact that the Fourier transform in (4.17) is controlled by the $S$-matrix of the final $g g$ dipole.

The last ingredient that we need in order to evaluate eq. (4.7) is the action of the transverse derivatives like $\left(u^{i}+i \partial_{x}^{i} / k^{+}\right)$. These will be shortly computed, but their effect can be anticipated on physical grounds: from the construction of the amplitude in eq. (3.17), we recall that the derivative $\partial_{x}^{i}$ acts on the gluon propagator at the emission point. Hence, the operator $\left(u^{i}+i \partial_{x}^{i} / k^{+}\right)$measures the difference between the transverse orientations of

\footnotetext{
${ }^{10}$ Very small values $0<x^{+}, y^{+}<\omega / Q_{s}^{2} \ll \tau_{f}$ corresponds to vacuum-like emissions with relatively large momenta $k_{\perp} \gtrsim Q_{s}$. Values close to $L^{+}$, such that $L^{+}-\tau_{f}<x^{+}, y^{+}<L^{+}$, yield boundary terms which cancel when summing up together the (in, in), (in, out), and (out, out) contributions.
} 
the source and of the emitted gluon, at the time of formation. Then, clearly, we expect its magnitude to be of order $\theta_{f} \equiv k_{f} / \omega$ (the formation angle introduced in eq. (2.7)). To check that, we shall compute

$$
\left(u^{i}+i \partial_{x}^{i} / k^{+}\right)\left(u^{i}-i \partial_{y}^{i} / k^{+}\right) \mathcal{K}_{q g}\left(x^{+}, \boldsymbol{b}_{\perp}+\boldsymbol{x}_{\perp} ; y^{+}, \boldsymbol{y}_{\perp} ; k^{+}\right)
$$

with the derivatives evaluated at $\boldsymbol{x}_{\perp}=\boldsymbol{u}_{\perp} x^{+}$and $\boldsymbol{y}_{\perp}=\boldsymbol{u}_{\perp} y^{+}$. The general expression for $\mathcal{K}_{q g}$ which is required for that purpose is given in the appendix. But for a parametric estimate, one can replace $\mathcal{K}_{q g}$ by the free propagator $\mathcal{G}_{0}$. We thus deduce

$$
\begin{aligned}
\left(u^{i}-\frac{i \partial_{y}^{i}}{k^{+}}\right)\left(u^{i}+\frac{i \partial_{x}^{i}}{k^{+}}\right) \mathcal{G}_{0} & =\left(u^{i}-\frac{i \partial_{y}^{i}}{k^{+}}\right)\left[\left(u^{i}-k^{+} \frac{b^{i}+x^{i}-y^{i}}{x^{+}-y^{+}}\right) \mathcal{G}_{0}\right] \\
& =\left[\frac{b_{\perp}^{2}}{\left(x^{+}-y^{+}\right)^{2}}+\frac{2 i}{k^{+}\left(x^{+}-y^{+}\right)}\right] \mathcal{G}_{0}
\end{aligned}
$$

where the last equality is obtained after setting $x^{i}-y^{i}=u^{i}\left(x^{+}-y^{+}\right)$. Using $x^{+}-y^{+} \sim \tau_{f}$ and $b_{\perp} \sim 1 / Q_{s}$, it is easy to check that the second term in the square brackets is the dominant one and is of order $1 /\left(k^{+} \tau_{f}\right) \sim \theta_{f}^{2}$, as anticipated.

We are finally in a position to estimate the spectrum (4.7) for direct emissions. To that aim, one has to multiply the Gaussian in eq. (4.17) by a factor $L^{+} \tau_{f}$ coming from the integrals over the time variables $y^{+}$and $x^{+}$(this factor is the longitudinal phase-space for medium-induced gluon radiation), by the factor $\theta_{f}^{2}$ which estimates the effects of the transverse derivatives, by a factor $k^{+} /\left(x^{+}-y^{+}\right) \sim \omega / \tau_{f}$ coming from the normalization of $\mathcal{G}_{0}$ in eq. (4.12) and, finally, by the overall factor $g^{2} C_{F}$ manifest on eq. (4.7). Putting all that together, one finds

$$
\mathcal{P}_{q}^{(\mathrm{in})}\left(\omega, \boldsymbol{k}_{\perp}\right) \propto \alpha_{s} C_{F} \theta_{f}^{2} L^{+} \frac{\omega}{Q_{s}^{2}} \exp \left\{-\frac{\left(\boldsymbol{k}_{\perp}-k^{+} \boldsymbol{u}_{\perp}\right)^{2}}{Q_{s}^{2}}\right\} .
$$

Eq. (4.21) is indeed the expected parametric estimate for the BDMPS-Z spectrum of the medium-induced radiation by a quark. A perhaps more familiar form of this spectrum is obtained by using eq. (2.7) for $\theta_{f}$ to deduce (for $\boldsymbol{u}_{\perp}=0$ )

$$
\omega \frac{\mathrm{d} N}{\mathrm{~d} \omega \mathrm{d} k_{\perp}^{2}} \propto \frac{\alpha_{s} C_{F}}{\sqrt{\omega \hat{q}}} \exp \left\{-\frac{k_{\perp}^{2}}{Q_{s}^{2}}\right\}
$$

It is here understood that $k_{\perp} \gtrsim k_{f} \simeq(\omega \hat{q})^{1 / 4}$, since the gluon acquires a transverse momentum of order $k_{f}$ already by the time of formation. The spectrum (4.22) is roughly flat in the range $k_{f}<k_{\perp}<Q_{s}$ and it is exponentially suppressed at larger values $k_{\perp} \gg Q_{s}$. After integration over $k_{\perp}$ and recalling that $Q_{s}^{2}=\hat{q} L^{+} \gg k_{f}^{2}$ and $\omega_{c}=\hat{q} L^{2} / 2$, this yields

$$
\omega \frac{\mathrm{d} N}{\mathrm{~d} \omega} \propto \alpha_{s} C_{F} \sqrt{\frac{\omega_{c}}{\omega}} \Longrightarrow \Delta E \equiv \int_{0}^{\omega_{c}} \omega \frac{\mathrm{d} N}{\mathrm{~d} \omega} \propto \alpha_{s} C_{F} \omega_{c}
$$

where the integration has been restricted to the phase-space for medium-induced radiation, i.e. $\omega_{\min }<\omega \leq \omega_{c}$, cf. eq. (2.9) (but the lower limit is irrelevant for computing the total energy loss, which is dominated by the upper limit $\omega_{c}$ ). 


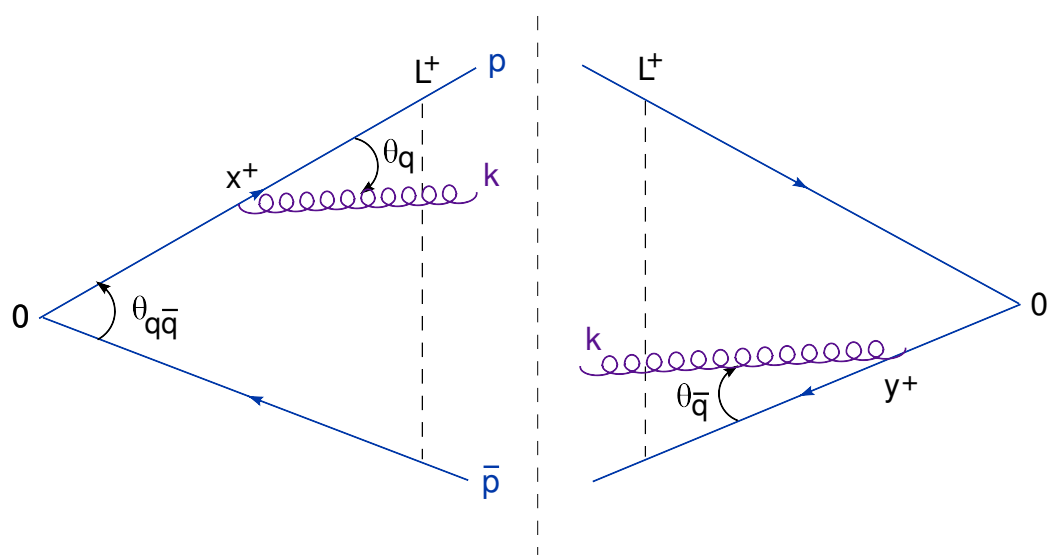

Figure 6. A Feynman graph for interference (amplitude times the complex conjugate amplitude).

It is finally interesting to compare the spectrum (4.22) for medium-induced radiation to the bremsstrahlung spectrum in eq. (2.2), for the same kinematics. By inspection of these equations, it is apparent that the medium-induced spectrum is formally the same (for any $k_{\perp}$ within the range $k_{f}<k_{\perp}<Q_{s}$ ) as the vacuum spectrum evaluated at $k_{\perp}=k_{f}$. Hence, clearly,

$$
\frac{\mathcal{P}_{q}^{(\mathrm{in})}}{\mathcal{P}_{q}^{\text {(vac) }}} \sim \frac{k_{\perp}^{2}}{\sqrt{\omega \hat{q}}} \sim \frac{k_{\perp}^{2}}{k_{f}^{2}},
$$

which shows that the medium-induced radiation dominates over bremsstrahlung for all the relevant momenta. This ratio is largest for $k_{\perp} \simeq Q_{s}$, when it becomes

$$
\frac{\mathcal{P}_{q}^{(\mathrm{in})}}{\mathcal{P}_{q}^{(\mathrm{vac})}} \sim \frac{L^{+}}{\tau_{f}} \sim \sqrt{\frac{\omega_{c}}{\omega}} \gg 1 \quad \text { for } \quad k_{\perp} \simeq Q_{s} .
$$

Physically, this is so because a gluon which is formed via medium rescattering can be emitted at any place inside the medium $\left(x^{+}, y^{+} \lesssim L^{+}\right)$, in contrast to the vacuum-like emissions, which are restricted to relatively short distances/times $x^{+}, y^{+} \lesssim \tau_{q} \ll L^{+}$. Accordingly, the longitudinal phase-space $L^{+} \tau_{f}$ for medium-induced radiation is parametrically larger than the corresponding phase-space $\sim \tau_{q}^{2}$ for bremsstrahlung.

\section{Medium-induced gluon radiation: interference terms}

We now turn to the main problem of interest for us in this paper, namely the contribution of the quark-antiquark interference to the medium-induced gluon radiation (see figure 6). Once again, we shall focus on the (in, in) piece, where the gluons are emitted inside the medium in both the direct and the complex conjugate amplitude. The respective contribution to the gluon spectrum is obtained by multiplying the quark amplitude (3.17) by the complex conjugate of the corresponding antiquark amplitude, performing the average over the medium and the sum (average) over the final (initial) color indices. 


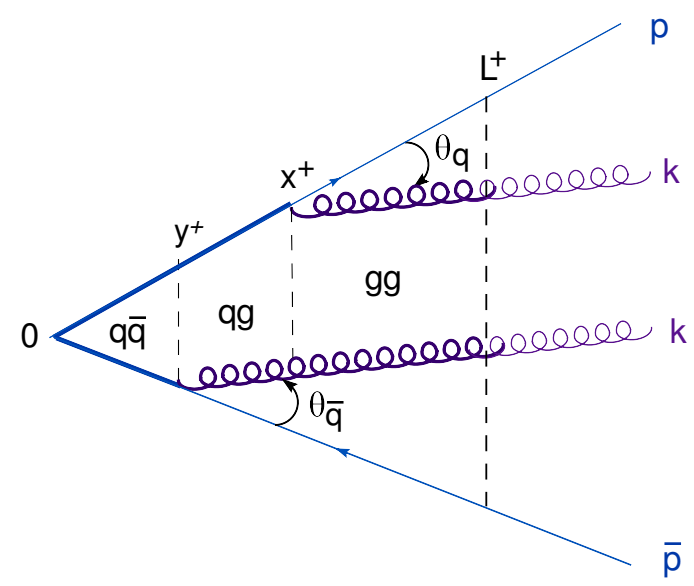

Figure 7. A folded version of the Feynman graph for interference where the amplitude and the complex conjugate amplitude are represented on top of each other, to more clearly exhibit the $q \bar{q}$, $q g$ and $g g$ dipoles. The (quark and gluon) Wilson lines are indicated with thick lines.

This yields

$$
\begin{aligned}
\mathcal{I}^{(\text {in })}(\boldsymbol{k})= & -2 g^{2} C_{F} \operatorname{Re} \int_{0}^{L^{+}} \mathrm{d} x^{+} \int_{0}^{x^{+}} \mathrm{d} y^{+} \mathrm{e}^{i k^{+}\left(u^{-} x^{+}-\bar{u}^{-} y^{+}\right)} \\
& \times \int \mathrm{d} \boldsymbol{z}_{1 \perp} \int \mathrm{d} \boldsymbol{z}_{2 \perp} \mathrm{e}^{-i \boldsymbol{k}_{\perp} \cdot\left(\boldsymbol{z}_{1 \perp}-\boldsymbol{z}_{2 \perp}\right)}\left(u^{i}+i \partial_{x}^{i} / k^{+}\right)\left(\bar{u}^{i}-i \partial_{y}^{i} / k^{+}\right) \\
& \times \frac{1}{N_{c}^{2}-1}\left\langle\operatorname{Tr} \mathcal{G}\left(L^{+}, \boldsymbol{z}_{1 \perp} ; x^{+}, \boldsymbol{x}_{\perp} ; k^{+}\right) \mathcal{U}_{q}\left(x^{+}, 0\right) \mathcal{U}_{\bar{q}}^{\dagger}\left(y^{+}, 0\right) \mathcal{G}^{\dagger}\left(L^{+}, \boldsymbol{z}_{2 \perp} ; y^{+}, \boldsymbol{y}_{\perp} ; k^{+}\right)\right\rangle, \\
& +(q \rightarrow \bar{q}),
\end{aligned}
$$

where the Wilson lines $\mathcal{U}_{q}\left(x^{+}, 0\right)$ and $\mathcal{U}_{\bar{q}}^{\dagger}\left(y^{+}, 0\right)$ refer to the quark and the antiquark, respectively, and it is understood that after performing the transverse derivatives $\partial_{x}^{i}$ and $\partial_{y}^{i}$ one has to identify $\boldsymbol{x}_{\perp}$ and $\boldsymbol{y}_{\perp}$ with the emission points $\boldsymbol{u}_{\perp} x^{+}$and $\overline{\boldsymbol{u}}_{\perp} y^{+}$, respectively. The explicit integrals in eq. (5.1) are written for the situation where the gluon is emitted at $x^{+}$ by the quark in the direct amplitude and absorbed by the antiquark at $y^{+}$in the complex conjugate amplitude, with $y^{+}<x^{+}$. The other possible configurations are obtained by exchanging the quark and the antiquark, as indicated in the last line of eq. (5.1). After 'folding' the Feynman graph as shown in figure 7, in such a way to superpose direct and conjugate amplitudes, one can view $y^{+}$as the 'first emission time', for an emission off the antiquark, and $x^{+}$as the 'second emission time', for an emission by the quark. Although somewhat formal, this perspective allows one to easily visualise the effective 'color dipoles' encoded in eq. (5.1), that we now discuss.

The subsequent manipulations are rather similar to those in section 4. Once again, one splits the quark Wilson line as $\mathcal{U}_{q}\left(x^{+}, 0\right)=\mathcal{U}_{q}\left(x^{+}, y^{+}\right) \mathcal{U}_{q}\left(y^{+}, 0\right)$ and one breaks the last gluon propagator into two pieces, from $y^{+}$to $x^{+}$and from $x^{+}$to $L^{+}$, by introducing an intermediate integration point $\boldsymbol{z}_{\perp}$. Then one uses the locality of the medium correlations in time to factorize the color trace into effective dipole contributions (cf. eq. (4.2)). This procedure now generates three dipole $S$-matrices: a quark-antiquark $(q \bar{q})$ dipole which 
extends in time from 0 up to $y^{+}$, a quark-gluon $(q g)$ dipole from $y^{+}$to $x^{+}$, and a gluongluon $(g g)$ dipole from $x^{+}$to $L^{+}$. The integrations over $\boldsymbol{z}_{1 \perp}$ and $\boldsymbol{z}_{2 \perp}$ are again performed as in eq. (4.5) and the outcome can be written as (compare to eq. (4.7))

$$
\begin{aligned}
\mathcal{I}^{(\mathrm{in})}(\boldsymbol{k})= & -2 g^{2} C_{F} \operatorname{Re} \int_{0}^{L^{+}} \mathrm{d} x^{+} \int_{0}^{x^{+}} \mathrm{d} y^{+} \mathrm{e}^{i k^{+}\left(u^{-} x^{+}-\bar{u}^{-} y^{+}\right)}\left(u^{i}+i \partial_{x}^{i} / k^{+}\right)\left(\bar{u}^{i}-i \partial_{y}^{i} / k^{+}\right) \\
& \times S_{q \bar{q}}\left(y^{+}, 0\right) \int \mathrm{d} \boldsymbol{z}_{\perp} \mathrm{e}^{-i \boldsymbol{k}_{\perp} \cdot\left(\boldsymbol{x}_{\perp}-\boldsymbol{z}_{\perp}\right)} \mathcal{K}_{q g}\left(x^{+}, \boldsymbol{z}_{\perp} ; y^{+}, \boldsymbol{y}_{\perp} ; k^{+}\right) S_{g g}\left(L^{+}, x^{+} ; \boldsymbol{x}_{\perp}-\boldsymbol{z}_{\perp}\right) \\
& +(q \rightarrow \bar{q}),
\end{aligned}
$$

where it is understood that $\boldsymbol{x}_{\perp} \rightarrow \boldsymbol{u}_{\perp} x^{+}$and $\boldsymbol{y}_{\perp} \rightarrow \overline{\boldsymbol{u}}_{\perp} y^{+}$after taking the derivatives. The $q \bar{q}$ dipole is evaluated similarly to eq. (3.29):

$$
S_{q \bar{q}}\left(y^{+}, 0\right) \simeq \exp \left\{-\frac{1}{12} \hat{q} \rho\left(\boldsymbol{u}_{\perp}-\overline{\boldsymbol{u}}_{\perp}\right)^{2}\left(y^{+}\right)^{3}\right\} \simeq \exp \left\{-\frac{1}{24} \hat{q} \rho \theta_{q \bar{q}}^{2}\left(y^{+}\right)^{3}\right\} .
$$

(We have also used $\left(\boldsymbol{u}_{\perp}-\overline{\boldsymbol{u}}_{\perp}\right)^{2} \simeq \theta_{q \bar{q}}^{2} / 2$ for small angles.) The $q g$ dipole is now built with the quark line in the direct amplitude and the gluon emitted by the antiquark in the complex conjugate amplitude. The corresponding propagator $\mathcal{K}_{q g}$ is defined as in eqs. (4.3)-(4.4) but with different boundary conditions for the path integral (4.3), namely $\boldsymbol{r}_{\perp}\left(y^{+}\right)=\overline{\boldsymbol{u}}_{\perp} y^{+}$ and $\boldsymbol{r}_{\perp}\left(x^{+}\right)=\boldsymbol{z}_{\perp}$. Finally, the $g g$ dipole is given by eq. (4.6), as before.

There are several important differences between the interference term (5.2) and the corresponding expression (4.7) for the direct emission. Two of them are quite obvious:

(a) The presence of the initial $q \bar{q}$ dipole, which measures the color coherence between the quark and the antiquark at the time $y^{+}$of the first emission. This phenomenon introduces an upper limit on $y^{+}$(as anticipated in eq. (2.17)):

$$
y^{+} \lesssim \tau_{\text {coh }} \simeq\left(\frac{24}{\hat{q} \rho \theta_{q \bar{q}}^{2}}\right)^{1 / 3} \sim L^{+}\left(\frac{\theta_{c}}{\theta_{q \bar{q}}}\right)^{2 / 3} .
$$

Indeed, for larger values $y^{+} \gtrsim \tau_{\text {coh }}$, one has $S_{q \bar{q}}\left(y^{+}, 0\right) \ll 1$, meaning that the color coherence is washed out. The parametric estimate in the r.h.s. shows that $\tau_{\text {coh }} \ll L^{+}$ so long as $\theta_{q \bar{q}} \gg \theta_{c}$. (As before, in writing parametric estimates we neglect numerical factors and treat $\rho$ as a constant, conveniently reabsorbed into the definition of $\hat{q}$.)

(b) The fact that the $q g$ dipole starts at $y^{+}$with a non-zero transverse size $r_{0}$ equal to the separation between the quark and the antiquark at that time (the maximal size of the $q \bar{q}$ dipole): $r_{0}=\left|\boldsymbol{u}_{\perp}-\overline{\boldsymbol{u}}_{\perp}\right| y^{+} \sim \theta_{q \bar{q}} y^{+}$.

A third difference between direct and interference terms, which is perhaps less obvious at this stage but will play a major role for the final results, is the following:

(c) The vacuum-like phase $\mathrm{e}^{i k^{+}\left(u^{-} x^{+}-\bar{u}^{-} y^{+}\right)}$in eq. (5.2) is not compensated in the calculation of medium-induced radiation, in contrast to what happened for the direct emissions (recall the discussion after eq. (4.16)). Rather, there is a left-over phase which controls the quantum coherence between the two emitters (see eq. (5.10) below). 
By itself, the constraint (5.4) represents a strong limitation on the longitudinal phasespace for interference and shows that the interference terms are suppressed with respect to the direct emissions. However, it turns out that the limitation introduced by the quantum coherence, cf. point (c) above, can be even stronger, depending upon the value of $\theta_{q \bar{q}}$. To understand this, we now perform a more detailed analysis of eq. (5.2).

The first step consists in clarifying the formation time. From section 4 , we recall that this is controlled by the propagator $\mathcal{K}_{q g}$ of the quark-gluon dipole. In the present case, this propagator measures the (quantum and color) coherence between the gluon emitted by one of the emitters and the other emitter. The 'exact' expression of $\mathcal{K}_{q g}$ valid in the harmonic approximation will be given in the appendix. Here we shall merely use a combination of small-time and large-time approximations, like in eqs. (4.12)-(4.14). The time scale separating between the two regimes is, once again, $\tau_{f}=1 /|\Omega|$, cf. eq. (4.11).

For small $x^{+}-y^{+} \ll \tau_{f}, \mathcal{K}_{q g}$ can be approximated by the saddle point approximation to the path integral in eq. (4.3), with the saddle point determined by the kinetic term alone. The corresponding classical path is readily determined as

$$
\boldsymbol{r}_{\text {class }}\left(z^{+}\right)-\boldsymbol{u}_{\perp} z^{+}=\boldsymbol{r}_{0}+\frac{z^{+}-y^{+}}{x^{+}-y^{+}}\left(z_{\perp}-\boldsymbol{u}_{\perp} x^{+}+\boldsymbol{r}_{0}\right), \quad \boldsymbol{r}_{0} \equiv\left(\overline{\boldsymbol{u}}_{\perp}-\boldsymbol{u}_{\perp}\right) y^{+} .
$$

As in section 4 , it is convenient to change variables from $\boldsymbol{z}_{\perp}$ (the gluon transverse position at time $x^{+}$) to $\boldsymbol{b}_{\perp} \equiv \boldsymbol{z}_{\perp}-\boldsymbol{u}_{\perp} x^{+}$(the final size of the $q g$ dipole and hence also the size of the $g g$ dipole at any time $z^{+} \geq x^{+}$). Then the saddle point (5.5) yields $\mathcal{K}_{q g} \approx \mathcal{G}_{0} S_{q g}$, with

$$
\begin{aligned}
\mathcal{G}_{0}\left(x^{+}, \boldsymbol{z}_{\perp} ; y^{+}, \overline{\boldsymbol{u}}_{\perp} y^{+} ; k^{+}\right) & =\frac{k^{+}}{2 \pi i\left(x^{+}-y^{+}\right)} \exp \left\{-i \frac{k^{+}\left(\boldsymbol{b}_{\perp}+\boldsymbol{u}_{\perp} x^{+}-\overline{\boldsymbol{u}}_{\perp} y^{+}\right)^{2}}{2\left(x^{+}-y^{+}\right)}\right\}, \\
S_{q g}\left(x^{+}, y^{+} ; \boldsymbol{b}_{\perp}\right) & \approx \exp \left\{-\frac{1}{12} \hat{q}\left(x^{+}-y^{+}\right)\left(b_{\perp}^{2}+r_{0}^{2}+\boldsymbol{b}_{\perp} \cdot \boldsymbol{r}_{0}\right)\right\}
\end{aligned}
$$

For larger time difference, $x^{+}-y^{+} \gg \tau_{f}$, the $q g$ dipole is exponentially suppressed, as manifest on eq. (4.14): $\mathcal{K}_{q g} \propto \mathrm{e}^{-\left(x^{+}-y^{+}\right) / \tau_{f}}$.

So, clearly, the actual formation time cannot be larger than $\tau_{f}$. However, depending upon the value of $r_{0} \sim \theta_{q \bar{q}} y^{+}$, this time could be shorter — that would be the case if the exponent in eq. (5.7) could become of order one already for $x^{+}-y^{+} \ll \tau_{f}$. To find out what is the actual scenario, one needs to consider $\mathcal{K}_{q g}$ simultaneously with the other constraints on the time integrations in eq. (5.2), which are specific to the interference problem. The first one is the condition for color coherence between the two emitters, as expressed by eq. (5.4). The second one is the condition for their quantum coherence, as encoded in the phase $\mathrm{e}^{i k^{+}\left(u^{-} x^{+}-\bar{u}^{-} y^{+}\right)}$manifest in eq. (5.2) together with a similar phase encoded in $\mathcal{K}_{q g}$ (see below).

To better appreciate the role of these phases, let us first consider the vacuum limit of the present calculation. In the vacuum, all the dipole $S$-matrices are set to one, the function $\mathcal{K}_{q g}$ reduces to the free gluon propagator $\mathcal{G}_{0}$, and then the integral over $\boldsymbol{z}_{\perp}$ in eq. (5.2) is straightforward. Using the integration variable $\boldsymbol{b}_{\perp} \equiv \boldsymbol{z}_{\perp}-\boldsymbol{u}_{\perp} x^{+}$, one finds

$$
\mathrm{e}^{i k^{+}\left(u^{-} x^{+}-\bar{u}^{-} y^{+}\right)} \int \mathrm{d} \boldsymbol{b}_{\perp} \mathrm{e}^{i \boldsymbol{b}_{\perp} \cdot \boldsymbol{k}_{\perp}} \mathcal{G}_{0}\left(x^{+}-y^{+} ; \boldsymbol{b}_{\perp}+\boldsymbol{u}_{\perp} x^{+}-\overline{\boldsymbol{u}}_{\perp} y^{+}\right)=\mathrm{e}^{i(k \cdot u) x^{+}-i(k \cdot \bar{u}) y^{+}}
$$


where the r.h.s. is recognized as the product of phases controlling the in-vacuum emission times, for the quark and the antiquark respectively. These phases imply $x^{+} \lesssim \tau_{q}$ and $y^{+} \lesssim \tau_{\bar{q}}$, where we recall that $\tau_{q} \propto 1 /(k \cdot u) \sim 1 / \omega \theta_{q}^{2}$ and similarly for $\tau_{\bar{q}}$. Then the time integrations generate the expected longitudinal phase-space $\tau_{q} \tau_{\bar{q}}$ for interference in the vacuum.

In the medium, the integral over $\boldsymbol{b}_{\perp}$ is controlled by the $S$-matrix $S_{g g}\left(L^{+}, x^{+} ; \boldsymbol{b}_{\perp}\right)$ of the $g g$ dipole, which enforces a rather small value $b_{\perp} \sim 1 / Q_{s}$. One can then replace $\mathcal{K}_{q g} \approx \mathcal{G}_{0}$ for the purposes of the $\boldsymbol{b}_{\perp}$-integration, which thus amounts to

$$
\begin{gathered}
\mathrm{e}^{i k^{+}\left(u^{-} x^{+}-\bar{u}^{-} y^{+}\right)} \int \mathrm{d} \boldsymbol{b}_{\perp} \mathrm{e}^{i \boldsymbol{b}_{\perp} \cdot \boldsymbol{k}_{\perp}} \exp \left\{-i \frac{k^{+}\left(\boldsymbol{b}_{\perp}+\boldsymbol{u}_{\perp} x^{+}-\overline{\boldsymbol{u}}_{\perp} y^{+}\right)^{2}}{2\left(x^{+}-y^{+}\right)}\right\} \exp \left\{-\frac{1}{4} Q_{s}^{2} b_{\perp}^{2}\right\} \\
\sim \mathrm{e}^{i \Phi} \frac{1}{Q_{s}^{2}} \exp \left\{-\frac{1}{Q_{s}^{2}}\left(\boldsymbol{k}_{\perp}-k^{+} \frac{\boldsymbol{u}_{\perp} x^{+}-\overline{\boldsymbol{u}}_{\perp} y^{+}}{x^{+}-y^{+}}\right)^{2}\right\}
\end{gathered}
$$

with the phase

$$
\Phi \equiv k^{+}\left(u^{-} x^{+}-\bar{u}^{-} y^{+}\right)-\frac{k^{+}\left(\boldsymbol{u}_{\perp} x^{+}-\overline{\boldsymbol{u}}_{\perp} y^{+}\right)^{2}}{2\left(x^{+}-y^{+}\right)}=-\frac{k^{+}\left(\boldsymbol{u}_{\perp}-\overline{\boldsymbol{u}}_{\perp}\right)^{2} x^{+} y^{+}}{2\left(x^{+}-y^{+}\right)} .
$$

In these manipulations, we have anticipated that $k^{+} /\left(x^{+}-y^{+}\right) \sim k_{f}^{2} \ll Q_{s}^{2}$ and we have used $\boldsymbol{u}_{\perp}^{2}=2 u^{-}$. At this point, one should recall that in the corresponding calculation for direct emissions, eqs. (4.16)-(4.17), the analog of this phase $\Phi$ has exactly canceled. The phase (5.10) is not quite the same as it would be in the vacuum, cf. eq. (5.8): it does not constrain the $x^{+}$and $y^{+}$variables individually, but a particular combination of them.

To summarize, the integrations over $x^{+}$and $y^{+}$are controlled by the following product

$$
\exp \left\{-i \frac{k^{+} \theta_{q \bar{q}}^{2} x^{+} y^{+}}{4\left(x^{+}-y^{+}\right)}\right\} \exp \left\{-\frac{1}{24} \hat{q}\left(x^{+}-y^{+}\right)\left(\theta_{q \bar{q}} y^{+}\right)^{2}\right\} \exp \left\{-\frac{x^{+}-y^{+}}{\tau_{f}}\right\}
$$

together with the constraint (5.4) coming from color coherence. The first factor in eq. (5.11) is the 'vacuum-like' phase $\Phi$. The second factor comes from the $S$-matrix (5.7) of the $q g$ dipole, where we have neglected $b_{\perp} \sim 1 / Q_{s}$ next to $r_{0} \sim \theta_{q \bar{q}} y^{+}$. The third factor is of course the large-time decay of the $q g$ propagator.

The four constraints introduced by the three factors in eq. (5.11) together with eq. (5.4) have to be simultaneously considered. Their analysis becomes streamlined if one first identifies the characteristic times scales associated with each of them. Let us enumerate these scales here:

(i) The color coherence time $\tau_{\text {coh }}$ : this is the maximal value of the first emission time $y^{+}$at which the quark and the antiquark do still form a color singlet. This scale is shown in eq. (5.4).

(ii) The in-medium formation time (here in the context of interference): this is the typical time interval $x^{+}-y^{+}$during which the $q g$ dipole loses quantum and color coherence. As we shall shortly argue, this scale is still determined by the last factor in eq. (5.11), like for direct emissions, and thus is equal to $\tau_{f}$, cf. eq. (2.4). 
(iii) The transverse resolution time $\tau_{\lambda}$ : this represents the characteristic time scale for quantum coherence between the two emitters during the process of gluon formation. This scale is determined by the phase in the first factor in eq. (5.11): the condition $\Phi \lesssim 1$ together with the fact that $x^{+}-y^{+} \sim \tau_{f}$ implies the following constraint on the emission times $x^{+}$and $y^{+}$:

$$
x^{+} y^{+} \lesssim \frac{4 \tau_{f}}{k^{+} \theta_{q \bar{q}}^{2}} \Longrightarrow \sqrt{x^{+} y^{+}} \lesssim \frac{\lambda_{f}}{\theta_{q \bar{q}}} \equiv \tau_{\lambda}
$$

where $\lambda_{f}=1 / k_{f}$ is the transverse wavelength of the gluon at the time of formation (we have used $\tau_{f} \sim k^{+} / k_{f}^{2}$ ). Some useful estimates for $\tau_{\lambda}$ have been given in eq. (2.14).

Eq. (5.12) represents in an average way the condition that the gluon overlap with both sources during the formation time. Since $x^{+} \simeq y^{+}+\tau_{f}$, it is clear that this condition must be viewed as a constraint on the first emission time $y^{+}$.

(iv) The interference time $\tau_{\text {int }}$ : as discussed in section 2.2 , this is the upper limit on $y^{+}$ which follows from eq. (5.12) in the large angle regime where $\theta_{q \bar{q}} \gg \theta_{f}$ (and hence $\left.\tau_{\lambda} \ll \tau_{f}\right)$ :

$$
y^{+} \lesssim \tau_{\text {int }} \equiv \frac{\tau_{\lambda}^{2}}{\tau_{f}}=\frac{2}{\omega \theta_{q \bar{q}}^{2}} .
$$

Some useful estimates for $\tau_{\text {int }}$ are shown in eq. (5.13). In the other interesting regime at small angles $\theta_{q \bar{q}} \ll \theta_{f}$ (or $\tau_{\lambda} \gg \tau_{f}$ ), the upper limit on $y^{+}$is essentially $\tau_{\lambda}$ (see section 2.2 for details).

Above, we have implicitly assumed that the original size $r_{0} \sim \theta_{q \bar{q}} y^{+}$of the $q g$ dipole does not to influence the formation time. Let us now check that this is indeed the case. The exponent in the middle factor in eq. (5.11) becomes of order one when $x^{+}-y^{+} \sim \tau_{f}$ and $y^{+} \sim \tau_{\lambda}$ (we have used $\hat{q} \tau_{f} \simeq k_{f}^{2}$ ). Since $y^{+}$cannot become larger than $\tau_{\lambda}$, as clear from eq. (5.12), we conclude that the original dipole size $r_{0}$ plays at most a marginal role in the formation process and thus cannot modify the formation time to parametric accuracy.

We have thus recognized in our calculation all the characteristic time scales for color and quantum coherence that were previously introduced, via physical considerations, in section 2.2. The interplay between these scales leads to the various regimes for interference identified in section 2.2 , that we shall not repeat here. Rather, we shall now explicitly check the arguments in section 2.2 concerning the suppression of the interference effects relative to the direct emissions.

To that aim, we shall estimate the contribution of the interference terms to the spectrum for medium-induced radiation for dipole angles $\theta_{q \bar{q}} \gg \theta_{c}$. This contribution is obtained by multiplying the Gaussian in eq. (5.9) by the corresponding longitudinal phasespace $\tau_{\min } \tau_{f}$, by the normalization $k^{+} /\left(x^{+}-y^{+}\right) \sim \omega / \tau_{f}$ of the gluon propagator (5.6), and by a factor $\theta_{f}^{2}$ which estimates the transverse derivatives in eq. (5.2). As in section 2.2, $\tau_{\min } \equiv \min \left(\tau_{\text {int }}, \tau_{\text {coh }}\right)$ is the smallest among the coherence scales which limit $y^{+}$in the context of interference: $\tau_{\min }=\tau_{\text {int }}$ when $\theta_{q \bar{q}} \gtrsim \theta_{f}$ and, respectively, $\tau_{\min }=\tau_{\text {coh }}$ when 
$\theta_{c} \ll \theta_{q \bar{q}} \lesssim \theta_{f}$. The angular factor $\theta_{f}^{2}$ is the same as for direct emissions, as it will be shortly checked. Putting all this together, one finds

$$
\mathcal{I}^{(\mathrm{in})}\left(\omega, \boldsymbol{k}_{\perp}\right) \propto-\alpha_{s} C_{F} \theta_{f}^{2} \tau_{\min } \frac{\omega}{Q_{s}^{2}} \exp \left\{-\frac{\left(\boldsymbol{k}_{\perp}-\Delta \boldsymbol{k}_{\perp}\right)^{2}}{Q_{s}^{2}}\right\} .
$$

The off-set $\boldsymbol{\Delta} \boldsymbol{k}_{\perp}$ in the Gaussian is obtained from the respective quantity in eq. (5.6) after averaging over the emission times:

$$
\Delta \boldsymbol{k}_{\perp}=k^{+}\left\langle\frac{\boldsymbol{u}_{\perp} x^{+}-\overline{\boldsymbol{u}}_{\perp} y^{+}}{x^{+}-y^{+}}\right\rangle \simeq k^{+} \boldsymbol{u}_{\perp}+k^{+}\left(\boldsymbol{u}_{\perp}-\overline{\boldsymbol{u}}_{\perp}\right) \frac{\tau_{\min }}{\tau_{f}} .
$$

Since $\tau_{\min } / \tau_{f} \ll 1$, it is clear that the second term in the r.h.s. is negligible as compared to the first one. Hence the ensuing off-set $\Delta \boldsymbol{k}_{\perp} \simeq k^{+} \boldsymbol{u}_{\perp}$ is the same as for direct emissions by the quark, cf. eq. (4.21). This is in agrement with our physical picture that, in order to allow for interferences, the gluon emitted by the antiquark must be co-moving with the quark (cf. figure 3 right). Clearly, for the reversed situation, where the gluon is emitted by the quark (and thus is co-moving with the antiquark), one would obtain $\Delta \boldsymbol{k}_{\perp} \simeq k^{+} \overline{\boldsymbol{u}}_{\perp}$.

By taking the ratio between the interference term (5.14) and the spectrum (4.21) for a direct emission by the quark, one finds, for $k_{f} \lesssim k_{\perp} \lesssim Q_{s}$,

$$
\mathcal{R}\left(\omega, \boldsymbol{k}_{\perp}\right) \equiv \frac{\left|\mathcal{I}^{(\mathrm{in})}\right|}{\mathcal{P}_{q}^{(\mathrm{in})}} \simeq \frac{\tau_{\text {min }}}{L},
$$

which in turn implies

$$
\left(\frac{\omega}{\omega_{c}}\right)^{1 / 2} \gtrsim \mathcal{R}\left(\omega, k_{\perp}\right) \gtrsim \frac{\omega}{\omega_{c}} \quad \text { when } \quad \theta_{f} \lesssim \theta_{q \bar{q}} \lesssim \theta_{s},
$$

for relatively large dipole angles $\theta_{q \bar{q}} \gtrsim \theta_{f}$ where $\tau_{\text {min }}=\tau_{\text {int }}$, and respectively

$$
1 \gg \mathcal{R}\left(\omega, k_{\perp}\right) \gtrsim\left(\frac{\omega}{\omega_{c}}\right)^{1 / 2} \quad \text { when } \quad \theta_{c} \ll \theta_{q \bar{q}} \lesssim \theta_{f},
$$

for smaller angles, $\theta_{c} \lesssim \theta_{f}$, where $\tau_{\text {min }}=\tau_{\text {coh. }}$. Eqs. (5.16)-(5.18) explicitly show the suppression of the interference effects relative to the direct emissions for the mediuminduced radiation of the dipole and confirm the respective estimates in section 2.2.

To complete the argument, one still needs to evaluate the transverse derivatives appearing in eq. (5.2), that is

$$
\left(u^{i}+i \partial_{x}^{i} / k^{+}\right)\left(\bar{u}^{i}-i \partial_{y}^{i} / k^{+}\right) \mathcal{K}_{q g}\left(x^{+}, \boldsymbol{b}_{\perp}+\boldsymbol{x}_{\perp} ; y^{+}, \boldsymbol{y}_{\perp} ; k^{+}\right)
$$

with the derivatives evaluated at $\boldsymbol{x}_{\perp}=\boldsymbol{u}_{\perp} x^{+}$and $\boldsymbol{y}_{\perp}=\overline{\boldsymbol{u}}_{\perp} y^{+}$. Like in the corresponding calculation for direct emission, eq. (4.19), we can replace $\mathcal{K}_{q g} \rightarrow \mathcal{G}_{0}$ to obtain a parametric estimate. This yields

$$
\begin{aligned}
\left(u^{i}+i \partial_{x}^{i} / k^{+}\right)\left(\bar{u}^{i}-i \partial_{y}^{i} / k^{+}\right) \mathcal{G}_{0} \rightarrow & \left(u^{i}-\frac{b^{i}+u^{i} x^{+}-\bar{u}^{i} y^{+}}{x^{+}-y^{+}}\right)\left(\bar{u}^{i}-\frac{b^{i}+u^{i} x^{+}-\bar{u}^{i} y^{+}}{x^{+}-y^{+}}\right) \\
& +\frac{2 i}{k^{+}\left(x^{+}-y^{+}\right)} .
\end{aligned}
$$


The last term in the r.h.s. if of order $1 /\left(k^{+} \tau_{f}\right) \sim \theta_{f}^{2}$. For small dipole angles $\theta_{q \bar{q}} \ll \theta_{f}$ it is easy to check that this is the dominant term. So, in what follows we focus on the less trivial case where $\theta_{q \bar{q}} \gg \theta_{f}$. Then one can use $y^{+} \simeq \tau_{\text {int }} \ll x^{+} \simeq \tau_{f}$ and $b_{\perp} \sim 1 / Q_{s}$ to simplify the algebra. The terms within the brackets in the r.h.s. of eq. (5.20) thus yield

$$
\frac{\left[b^{i}-\left(\bar{u}^{i}-u^{i}\right) y^{+}\right]\left[b^{i}-\left(\bar{u}^{i}-u^{i}\right) x^{+}\right]}{\left(x^{+}-y^{+}\right)^{2}} \simeq \frac{\boldsymbol{b}_{\perp} \cdot\left(\boldsymbol{u}_{\perp}-\overline{\boldsymbol{u}}_{\perp}\right)+\tau_{\text {int }}\left(\boldsymbol{u}_{\perp}-\overline{\boldsymbol{u}}_{\perp}\right)^{2}}{\tau_{f}} .
$$

Using $\left(\boldsymbol{u}_{\perp}-\overline{\boldsymbol{u}}_{\perp}\right)^{2} \sim \theta_{q \bar{q}}^{2}$ together with eq. (2.19), it becomes clear that the last term above is of order $\theta_{f}^{2}$. As for the first term, this is estimated as (after performing the $\boldsymbol{b}_{\perp}$-integration, cf. eq. (5.9))

$$
\frac{\left(\boldsymbol{k}_{\perp}-k^{+} \boldsymbol{u}_{\perp}\right) \cdot\left(\boldsymbol{u}_{\perp}-\overline{\boldsymbol{u}}_{\perp}\right)}{Q_{s}^{2} \tau_{f}} \lesssim \frac{\omega \theta_{q} \theta_{q \bar{q}}}{Q_{s}^{2} \tau_{f}} \sim \theta_{f}^{2} \frac{\theta_{q} \theta_{q \bar{q}}}{\theta_{s}^{2}} \lesssim \theta_{f}^{2} .
$$

We have also used here eq. (4.18) together with the relations $k_{f}^{2} \sim \omega / \tau_{f}$ and $k_{f} / Q_{s}=\theta_{f} / \theta_{s}$. To conclude, the dominant effect of the transverse derivatives in the interference terms is a factor $\theta_{f}^{2}$, so like for the direct emissions.

\section{Discussion and outlook}

Throughout this work, we have mostly focussed on medium-induced radiation of the BDMPS-Z type, whose main characteristic is that the gluons are emitted inside the medium, as a result of multiple scattering. However, we have also noticed at several places that this is not the only type of medium-induced radiation for the case of a dense medium. Indeed, as found in refs. [26, 27] (and reviewed in our section 3.2), the color decoherence of the $q \bar{q}$ antenna leads to additional radiation outside of the medium, which is localized in a region of (angular) phase space that would be forbidden — by destructive interference in the vacuum. The essential reason why this new type of radiation exists is because the characteristic time scale $\tau_{\text {coh }}$ beyond which the $q \bar{q}$ pair loses its color coherence becomes much smaller than the vacuum-like formation time for a gluon radiated outside the dipole cone, which is typically $\tau_{\text {int }}=2 /\left(\omega \theta_{q \bar{q}}^{2}\right)$. However, according to eq. (2.19), the inequality $\tau_{\text {coh }} \ll \tau_{\text {int }}$ holds whenever $\theta_{q \bar{q}} \ll \theta_{f}$, which allows for (vacuum) formation times $\tau_{\text {int }}$ that are both larger and smaller than the medium size $L$. Hence, the same mechanism could in principle lead to additional gluon radiation inside the medium. This possibility has not been mentioned in the previous literature, so we shall succinctly explore it here, via qualitative considerations based on our previous results.

Specifically, one has $\tau_{\text {int }} \simeq L$ when $\theta_{q \bar{q}} \simeq \theta_{\text {out }}$, where

$$
\theta_{\text {out }} \equiv \sqrt{\frac{2}{\omega L}}=\theta_{c} \sqrt{\frac{\omega_{c}}{\omega}}=\theta_{f}\left(\frac{\omega}{\omega_{c}}\right)^{1 / 4}
$$

is the same as the upper limit in our eq. (3.31). So, a priori there are two angular regions where the mechanism proposed in refs. [26, 27] could operate: ${ }^{11}$ (i) $\theta_{c} \ll \theta_{q \bar{q}} \lesssim \theta_{\text {out }}$, where $\tau_{\text {int }} \gtrsim L$, so the respective emissions take place outside the medium; this is the situation

\footnotetext{
${ }^{11}$ We recall that the lower limit $\theta_{c}$ on $\theta_{q \bar{q}}$ comes from the condition that $\tau_{\text {coh }} \ll L$, cf. eq. (2.18).
} 
considered in $[26,27]$ and (ii) $\theta_{\text {out }} \ll \theta_{q \bar{q}} \ll \theta_{f}$ where $\tau_{\text {int }} \ll L$, so the gluons are emitted (via vacuum-like processes) inside the medium; this is the new possibility that we would like to explore. Note that, together, these two angular domains completely overlap with our region of 'relatively small dipole angles' for BDMPS-Z radiation, as defined in section 2.2. This observation naturally leads to the following two questions: (a) what is the dominant mechanism for medium-induced radiation for dipole angles within this common range at $\theta_{c} \ll \theta_{q \bar{q}} \ll \theta_{f}$, and (b) what are the most interesting values of $\theta_{q \bar{q}}$ for applications to the phenomenology? These are the main questions that we would like to address in this section.

With respect to the first question above, its answer depends upon the ration $\theta_{q \bar{q}} / \theta_{\text {out }}$, as we argue now. When $\theta_{c} \ll \theta_{q \bar{q}} \lesssim \theta_{\text {out }}$, that is, in region (i) above, the radiation due to the new mechanism of refs. [26, 27] is concentrated outside the dipole cone, but relatively close to it: indeed, this radiation has the angular distribution of the usual bremsstrahlung spectrum, that is, it is strongly peaked around the sources and it decays as $1 / \theta$ at large emission angles $\theta \gg \theta_{q \bar{q}}$. By contrast, the BDMPS-Z gluons are emitted at relatively large angles $\theta_{f} \gg \theta_{\text {out }}$ w.r.t. their sources, meaning far outside the dipole cone. Hence in range (i) for $\theta_{q \bar{q}}$, both types of medium-induced radiation exist, but they are widely separated in angle from each other: the out-of-medium emissions dominate the spectrum for $\theta_{q}, \theta_{\bar{q}} \sim \theta_{q \bar{q}}$, while the in-medium emissions à la BDMPS-Z dominate for $\theta_{q}, \theta_{\bar{q}} \sim \theta_{f}$.

Consider now larger dipole angles, $\theta_{\text {out }} \ll \theta_{q \bar{q}} \ll \theta_{f}$ (the angular region (ii)). Then the previous discussion of the BDMPS-Z gluons goes unchanged, whereas the mechanism proposed in refs. $[26,27]$ is expected to become ineffective: indeed, in-medium radiation with small emissions angles $\theta_{q \bar{q}} \ll \theta_{f}$ and hence relatively large formation time $\tau_{\text {int }} \gg \tau_{f}$ is strongly suppressed as compared to the BDMPS-Z radiation, since the soft gluons cannot avoid accumulating transverse momenta of order $k_{f}$, via medium rescattering; as a consequence, they are liberated from the parent quark after a relatively short time $\tau_{f}$ and at an angle $\sim \theta_{f}$. Hence, for dipole angles within region (ii), the medium-induced radiation is predominantly of the BDMPS-Z type and is distributed at large angles $\theta \gtrsim \theta_{f} \gg \theta_{q \bar{q}}$, far outside the dipole cone.

We thus expect the physical consequences of the two mechanisms for medium-induced radiation to be quite different: whereas the BDMPS-Z gluons are more effective in broadening the energy distribution of a jet in the transverse plane, in qualitative agreement with the observations at the LHC $[2,3]$, the mechanism proposed in $[26,27]$ is probably more important for the softening of the intra-jet radiation and its redistribution at small angles. A more detailed phenomenological analysis is still needed before drawing firm conclusions on the last point.

Since the in-medium antenna pattern is so sensitive to the value of the dipole angle, it is important to estimate what are the relevant values in the context of heavy ion collisions. A physical process where in-medium, color-singlet, antennas like the one that we have discussed are naturally generated is the hadronic decay of a heavy vector boson like the $\mathrm{Z}$ or the W. In this scenario, the dipole angle of the pair depends upon the boson kinematics, in particular, on its boost: $\theta_{q \bar{q}} \sim 1 / \gamma$. However, while such bosons are copiously produced in $\mathrm{Pb}+\mathrm{Pb}$ collisions at the $\mathrm{LHC}$, their identification via hadronic decays is complicated, if at all possible, by the large QCD background. ${ }^{12}$

\footnotetext{
${ }^{12}$ We thank P. Quiroga, S. Sapeta and G. Soyez for useful discussions on this topic.
} 
Another source of in-medium antennas, but typically in non-singlet color representations, is the evolution of jets produced via hard processes in heavy collisions. Although our calculations have been restricted to the color singlet case, our arguments are sufficiently general to be applicable to a qualitative discussion of antennas in other representations. We expect that the physical regimes for interference summarized in section 2.2 will apply for any color representation. In particular, our main conclusion remains unchanged: for relatively large angles $\theta_{q \bar{q}} \gg \theta_{c}$, the interference effects are suppressed and the overall antenna pattern is the sum of two independent BDMPS-Z spectra produced by the two legs of the antenna. For smaller angles, on the other hand, the interference effects are important and their result is such that, when $\theta_{q \bar{q}} \ll \theta_{c}$, the total in-medium radiation by the antenna coincides with that by a single source which carries the global color charge of the antenna (e.g., a source in the adjoint representation if the antenna was produced by a gluon decay). It would be interesting to check this conclusion explicitly. (The calculation of the octet channel in [27] provides a partial check in that sense.)

Within the in-medium jet evolution we can distinguish two types of antennas: those generated via hard, vacuum-like, parton splittings and those arising via medium-induced emissions. Addressing the relevant angles in either case will ultimately resort on in-medium Monte-Carlo generators, such as [34-39], which can keep track of all the kinematical and probabilistic effects. Here we will provide some simple estimates based on physical arguments, to be ultimately confronted to explicit calculations. For simplicity, we shall treat $\hat{q}$ as a fixed parameter in these estimates, in lines with our general strategy throughout this paper.

Consider first an antenna resulting from medium-induced radiation. The main question is, what is the typical angle between the emitted gluon and the parent parton by the time of a subsequent gluon emission. This angle starts with a value $\sim \theta_{f}$ at the time of formation but it can be enlarged by additional multiple scattering occurring after the formation. So, we need to estimate the typical time interval $\tau_{\text {rad }}$ between two successive emissions. The probability for emitting a new gluon can be estimated as $\mathcal{P} \sim \alpha_{s} C_{R} n_{\text {eff }}$ where $n_{\text {eff }} \equiv \tau_{\text {rad }} / \tau_{f}$ is the number of effective scattering centers along $\tau_{\text {rad }}$. This probability becomes of $\mathcal{O}(1)$ when

$$
\tau_{\text {rad }} \sim \frac{\tau_{f}}{\alpha_{s} C_{R}} .
$$

This estimate is a bit simplistic, since emissions can happen at different frequencies and the relevant probability is the inclusive one. Since the number of emitted gluons grows with decreasing $\omega$, cf. eq. (2.8), a more realistic estimate (or, at least, a strict lower limit) reads

$$
\tau_{\mathrm{rad}}>\frac{\tau_{f}\left(\omega_{\min }\right)}{\alpha_{s} C_{R}}=\frac{\ell}{\alpha_{s} C_{R}},
$$

where $\omega_{\min }$ is the lowest energy for BDMPS-Z gluons, cf. eq. (2.6), and $\ell$ is the mean free path for elastic collisions. Thus, for gluon frequencies which are not parametrically larger (in $\alpha_{s}$ ) that $\omega_{\min }$, we have $\tau_{\text {rad }} \gg \tau_{f}(\omega)$ and the partons that form the antenna acquire a significant momentum by the time of secondary emissions, leading to an effective dipole angle much larger than $\theta_{f}$. Thus, medium-induced gluons lead, typically, to relatively 
large dipoles according to the classification of section 2.2. This implies that the interference effects can be safely ignored when studying the jet evolution via medium-induced radiation. Note also that for the medium-induced parton cascade, this radiation time $\tau_{\text {rad }}$ plays the role of an effective medium length. Hence that fact that $\tau_{\text {rad }} \gg \tau_{f}$ guarantees the validity of our central argument for the suppression of the interference terms (the suppression factor being $\tau_{f} / \tau_{\text {rad }}$ in this case).

A different source of antennas propagating in the medium is the vacuum-like evolution of hard partons. This refers to the emission of gluons with large transverse momenta $k_{\perp} \gg Q_{s}$ and hence very short formation times $\tau_{q} \ll \tau_{f}$ (for a given frequency). The precise values of the dipole opening depends on the kinematics of the intervening hard processes, in particular on their energy and virtuality. Indeed, for a hard parton of energy $E$ and virtuality $Q$ which emits a hard gluon carrying a fraction $z$ of its energy, the angle of emission is

$$
\theta_{\text {hard }}^{2} \approx \frac{1}{z(1-z)} \frac{Q^{2}}{E^{2}}
$$

and the emission time is estimated via the uncertainty principle as

$$
\tau_{\text {hard }} \sim \frac{E}{Q^{2}} \sim \frac{1}{z(1-z)} \frac{1}{\theta_{\text {hard }}^{2} E} .
$$

(Using $\omega \simeq z E$ for a small- $z$ emission and $\omega \theta_{\text {hard }} \simeq k_{\perp}$, it becomes clear that eq. (6.5) is consistent with our basic formula (2.1) for the formation time.) Thus, unless the branchings are very asymmetric, the vacuum-like evolution can produce antennas with very small angles and at very early times $\tau_{\text {hard }} \ll L$. We conclude that light jets (those with jet mass much smaller than their total energy) can be sources for all the different types of dipoles discussed in section 2.2, with a predilection though for small and very small dipoles in the sense of that discussion. Depending upon the precise relation between the emission angle $\theta_{\text {hard }}$ and the characteristic medium angle $\theta_{c}$, the antenna created via such a hard branching can either act as a set of two independent sources of BDMPS-Z gluons (if $\theta_{\text {hard }} \gg \theta_{c}$ ), or radiate such gluons in the same way as the parent parton would do (if $\theta_{\text {hard }} \lesssim \theta_{c}$ ).

So far, we have discussed the in-medium hard branchings only as a mechanism for generating antennas, but we have not addressed the medium effects on such a branching by itself. As a matter of facts, we do not expect such effects to be significant: the in-medium emissions of relatively hard gluons with transverse momenta $k_{\perp} \gg Q_{s}$ should proceed exactly as in the vacuum, for both direct emissions and the corresponding interference phenomena leading to angular ordering. This is quite clear from the fact that the respective formation time $\tau_{\text {hard }}$ is much shorter than the time scale $\tau_{\text {coh }}$ for the color decoherence of the sources. Since there was some confusion on this point in the recent literature [40], we would like to take this opportunity and fully clarify this issue. The analysis in ref. [40] was based on the assumption that the color decorrelation time (the analog of our $\tau_{\text {coh }}$ ) is to be identified with the mean free path $\ell$ of a colored parton propagating through the medium (as introduced in the discussion in section 2.1). That assumption would be correct if and only if the two emitters which form the antenna would undergo independent color rotations in the medium, which would be the case if their transverse separation $r_{\perp} \sim \tau_{q} \theta_{q \bar{q}}$ at the 
time of emission was larger than the Debye screening length $\mu_{D}^{-1}$. Clearly, this can only happen for extremely soft radiated gluons, with transverse momenta $k_{\perp} \simeq \omega \theta_{q \bar{q}} \lesssim \mu_{D}$. For the medium created in heavy ion collisions at the current energies, this scale $\mu_{D}$ is of the order of a few hundred MeV. Gluons with such momenta are truly soft and do not significantly contribute to the in-medium evolution of a hard jet, which rather proceeds via hard, vacuum-like, emissions and semi-hard, medium-induced, ones.

\section{Acknowledgments}

We would like to thank Al Mueller for insightful and patient explanations on the BDMPS-Z physics, and Peter Arnold, Andrei Leonidov, Cyrille Marquet, Guilherme Milhano, Carlos Salgado, and Urs Wiedemann for many related discussions and useful comments on the manuscript.

\section{A Momentum space analysis of the gluon spectrum}

The total radiation probability from the dipole, which includes the direct emissions from the quark, eq. (4.7), and the antiquark (as obtained by replacing $u \rightarrow \bar{u}$ within eq. (4.7)) and the quark-antiquark interference terms, eq. (5.2), can be compactly expressed as

$$
\begin{aligned}
& \mathcal{P}_{\text {tot }}^{(\text {in })}=2 g^{2} C_{F} \operatorname{Re} \sum_{F, L=q, \bar{q}} \operatorname{Sign}(F, L) \times \\
& \int_{0}^{L^{+}} \mathrm{d} x^{+} \int_{0}^{x^{+}} \mathrm{d} y^{+} \int \mathrm{d}^{2} \boldsymbol{b}_{\perp} \mathrm{e}^{i \boldsymbol{k}_{\perp} \cdot \boldsymbol{b}_{\perp}} S_{g g}\left(L^{+}, x^{+} ; \boldsymbol{b}_{\perp}\right) S_{F, L}\left(y^{+}, \mathbf{0}\right) I_{u_{L}, u_{F}}\left(x^{+}, y^{+}, \boldsymbol{b}_{\perp}\right)
\end{aligned}
$$

(Note a slight change in the notations for the quark 4-velocities as compared to the main text: we identify $u_{q} \equiv u$ and $u_{\bar{q}} \equiv \bar{u}$. To avoid cumbersome notations, we shall indicate the transverse components of $u_{F}$ and $u_{L}$ by boldface symbols without the ' $\perp$ ' subscript: $\boldsymbol{u}_{F}$ and $\left.\boldsymbol{u}_{L}.\right)$ The quark-quark dipole is trivial when both indexes are the same $\left(S_{q q}=S_{\bar{q} \bar{q}}=1\right)$ and the function $\operatorname{Sign}(F, L)=1$ if $F=L$ and $\operatorname{Sign}(F, L)=-1$ otherwise. $^{13}$ These four terms summarize the four possible combinations appearing in the total emission probability which are the direct emissions from either the quark or the antiquark (Fig 5) and the two interference terms in which the gluon is first emitted, at time $y^{+}$, by the fermion which has velocity $u_{F}$ and then absorbed, at time $x^{+}$, by the other fermion, with velocity $u_{L}$ (figure 7). The function $I_{u_{L}, u_{F}}\left(x^{+}, y^{+}, \boldsymbol{b}_{\perp}\right)$ encodes the quark-gluon dipole together with its transverse derivatives:

$$
\begin{aligned}
I_{u_{L}, u_{F}}\left(x^{+}, y^{+}, \boldsymbol{b}_{\perp}\right)= & \mathrm{e}^{i k^{+}\left(u_{L}^{-} x^{+}-u_{F}^{-} y^{+}\right)}\left(u_{L}^{i}+i \partial_{x}^{i} / k^{+}\right)\left(u_{F}^{i}-i \partial_{y}^{i} / k^{+}\right) \\
& \left.\mathcal{K}_{q g}\left(x^{+}, \boldsymbol{x}_{\perp}+\boldsymbol{b}_{\perp} ; y^{+}, \boldsymbol{y}_{\perp} ; k^{+}\right)\right|_{\boldsymbol{x}_{\perp}=\boldsymbol{u}_{L} x^{+}, \boldsymbol{y}_{\perp}=\boldsymbol{u}_{F} y^{+}}
\end{aligned}
$$

For the case of direct emissions, where $u_{L}$ and $u_{F}$ coincide with each other, the $q g$ dipole is made with the gluon and the quark which has emitted that gluon (the parent quark).

\footnotetext{
${ }^{13}$ Eq. (4.7) corresponds to the term $u_{L}=u_{F}=u$ and eq. (5.2) is obtained by setting $u_{L}=u$ and $u_{F}=\bar{u}$. A change of variables $\boldsymbol{b}_{\perp}=\boldsymbol{z}_{\perp}-\boldsymbol{x}_{\perp}$ has been also performed.
} 
For the interference terms, this dipole is made with the gluon emitted by the quark with velocity $u_{F}$ and the other quark, which has a velocity $u_{L}$.

We restrict ourselves to the 'harmonic approximation', where the slowly varying logarithm $\rho$ which enters the various dipole $S$-matrices (see e.g. eqs. (4.4) and (4.6)) is treated as a fixed quantity, reabsorbed into the normalization of $\hat{q}$. In this approximation, the quark-gluon path integral (4.3) is exactly known [11, 19] for the case of a single emitter with vanishing transverse velocity. The generalization to the present case, where the quark which enters the quark-gluon dipole possesses a non-zero transverse velocity $\boldsymbol{u}_{L}$, is easily to find and reads

$$
\begin{aligned}
\mathcal{K}_{q g}\left(x^{+}, \boldsymbol{x}_{\perp} ; y^{+}, \boldsymbol{y}_{\perp} ; k^{+}\right)= \\
\mathrm{e}^{-i k^{+} u_{L}^{-}\left(x^{+}-y^{+}\right)+i k^{+} \boldsymbol{u}_{L} \cdot(\boldsymbol{x}-\boldsymbol{y})_{\perp}} \\
\quad \times\left.\frac{A}{2 \pi} \exp \left\{-\frac{A}{2}\left(B\left(\overline{\boldsymbol{x}}_{\perp}^{2}+\overline{\boldsymbol{y}}_{\perp}^{2}\right)-2 \overline{\boldsymbol{x}}_{\perp} \cdot \overline{\boldsymbol{y}}_{\perp}\right)\right\}\right|_{\overline{\boldsymbol{x}}_{\perp}=\boldsymbol{x}_{\perp}-\boldsymbol{u}_{L} x^{+}, \overline{\boldsymbol{y}}_{\perp}=\boldsymbol{y}_{\perp}-\boldsymbol{u}_{L} y^{+}}
\end{aligned}
$$

where $\Omega$ has been already defined in eq. (4.8) and we have introduced

$$
A \equiv \frac{k^{+} \Omega}{i \sinh \Omega\left(x^{+}-y^{+}\right)}, \quad B \equiv \cosh \Omega\left(x^{+}-y^{+}\right) .
$$

\section{A.1 The gluon spectrum at the time of formation}

The quark-gluon dipole encodes the process of in-medium gluon formation. Right after formation, the gluon spectrum is obtained via the Fourier transform of eq. (A.2). After some lengthy but straightforward algebra, we obtain

$$
\begin{aligned}
& I_{u_{L}, u_{F}}\left(x^{+}, y^{+}, \boldsymbol{q}_{\perp}\right)= \frac{1}{B}\left[\left(\boldsymbol{v}_{\perp}-\boldsymbol{u}_{L}\right)^{2} \frac{1}{B}+\left(\boldsymbol{v}_{\perp}-\boldsymbol{u}_{L}\right) \cdot\left(\boldsymbol{u}_{L}-\boldsymbol{u}_{F}\right)\left(1+C \Omega y^{+}\right)\right] \\
& \times \exp \left\{-i C \frac{k^{+}\left(\boldsymbol{v}_{\perp}-\boldsymbol{u}_{L}\right)^{2}}{2 \Omega}+i \frac{k^{+}}{2}\left(\boldsymbol{u}_{L}-\boldsymbol{u}_{F}\right)^{2}\left(1+C \Omega y^{+}\right) y^{+}\right. \\
&\left.\quad+i \frac{k^{+}}{B}\left(\boldsymbol{v}_{\perp}-\boldsymbol{u}_{L}\right) \cdot\left(\boldsymbol{u}_{L}-\boldsymbol{u}_{F}\right) y^{+}\right\}
\end{aligned}
$$

where $\boldsymbol{v}_{\perp} \equiv \boldsymbol{q}_{\perp} / k^{+}$is the gluon transverse velocity when it is formed and $C \equiv \tanh \Omega\left(x^{+}-\right.$ $\left.y^{+}\right)$has the properties that $C / \Omega \simeq x^{+}-y^{+}$when $x^{+}-y^{+} \ll \tau_{f}$ and $C \rightarrow 1$ when $x^{+}-y^{+} \gg \tau_{f}$. (Recall that $\tau_{f} \equiv 1 /|\Omega|$, cf. eq. (4.11).)

The analysis of this expression shows the main features of the gluon spectrum at the time of formation. For $x^{+}-y^{+} \simeq \tau_{f}$, which is the typical value fixed by the subsequent integrations over $x^{+}$and $y^{+}$, one can write $C \Omega \approx 1 / \tau_{f}$ to parametric accuracy, and then the first term in the exponent is parametrically the same as

$$
\exp \left\{-i \frac{k^{+}\left(\boldsymbol{v}_{\perp}-\boldsymbol{u}_{L}\right)^{2}}{2 \tau_{f}}\right\}=\exp \left\{-i \frac{\left(\boldsymbol{q}_{\perp}-k^{+} \boldsymbol{u}_{L}\right)^{2}}{2 k_{f}^{2}}\right\} .
$$

Hence, for both the interference and the direct terms, the transverse momentum distribution is a Gaussian with width $k_{f}^{2} \sim \sqrt{\omega \hat{q}}$ centered around the direction $\boldsymbol{u}_{L}$ of the quark which 
enters the quark-gluon dipole. (Note that, in the interference term, this is not the quark which emitted the gluon, but the other quark.)

Concerning the angular structure of the spectrum, as encoded in the first line of eq. (A.5), this is a natural generalization of the corresponding result in the vacuum, to which it reduces, as it should, in the limit $|\Omega| \rightarrow 0$. Indeed, in that limit, $B \rightarrow 1, C \rightarrow 0$, so the expression within the square brackets becomes $\left(\boldsymbol{v}_{\perp}-\boldsymbol{u}_{L}\right) \cdot\left(\boldsymbol{v}_{\perp}-\boldsymbol{u}_{F}\right)$; this is the expected result for both the direct terms, cf. eq. (3.21), and the interference ones, cf. eq. (3.23). (Of course, in the vacuum, the gluon velocity at the time of formation is the same as its final velocity.) One can similarly check that, when $|\Omega| \rightarrow 0$, the exponent in eq. (A.5) reduces to the respective vacuum result, i.e., to the formation-time phases visible e.g. in eq. (5.8).

In addition to the angle and momentum distributions, eq. (A.5) also shows what are the time scales involved in the radiation process. The overall prefactor $1 / B$, which at long times behaves as $1 / B \sim \exp \left\{-\Omega\left(x^{+}-y^{+}\right)\right\}$, sets the formation time of the gluons as $x^{+}-y^{+} \simeq \tau_{f}$, in agreement with eq. (2.4). The other relevant time scale is the typical value of the first emission time $y^{+}$(more properly, this is the time at which the gluon formation is initiated). For direct emissions $\left(\boldsymbol{u}_{L}=\boldsymbol{u}_{F}\right)$, there is no characteristic value of $y^{+}$and emissions happen all along the medium length with equal probability. By contrast, for the interference terms $\left(\boldsymbol{u}_{L} \neq \boldsymbol{u}_{F}\right)$, the values of $y^{+}$are constrained by two new time scales, $\tau_{\lambda}$ and $\tau_{\text {int }}$, which are generated by the middle term in the exponent in eq. (A.5) and its interplay with the other terms.

Specifically, for $x^{+}-y^{+} \simeq \tau_{f}$, one can write $1+C \Omega y^{+} \approx\left(\tau_{f}+y^{+}\right) / \tau_{f} \approx x^{+} / \tau_{f}$ to parametric accuracy, and hence

$$
\frac{i k^{+}}{2}\left(\boldsymbol{u}_{L}-\boldsymbol{u}_{F}\right)^{2}\left(1+C \Omega y^{+}\right) y^{+} \approx i \frac{k^{+} \theta_{q \bar{q}}^{2} x^{+} y^{+}}{4 \tau_{f}} .
$$

This is clearly equivalent with the first factor in eq. (5.11). As explained in sections 2.2 and 5, this term encodes two time scales: $\tau_{\lambda}$, which is an upper limit on $\sqrt{y^{+}\left(y^{+}+\tau_{f}\right)}$ for generic values of $\theta_{q \bar{q}}$, cf. eq. (5.12), and $\tau_{\text {int }}$, which is the ensuing limit on $y^{+}$for relatively large angles $\theta_{q \bar{q}} \gg \theta_{f}$, cf. eq. (5.13). The last term in the exponent in eq. (A.5), which involves the momentum acquired by the gluon during formation, leads to the same time scale $\tau_{\lambda}$, as clear from the fact that $k^{+}\left|\boldsymbol{v}_{\perp}-\boldsymbol{u}_{L}\right| \sim \omega \theta_{f}$ for the typical gluon velocity $\boldsymbol{v}_{\perp}$. Finally, in addition to eq. (A.5), the time dependence of the interference term is also sensitive to the overall suppression due to the initial $q \bar{q}$ dipole, eq. (5.3), which introduces the additional time scale $\tau_{\text {coh }}$ for color decoherence.

At this level, it is straightforward to make contact between eq. (A.5) and the expression (2.8) for the 'formation' spectrum deduced in section 2.2 via qualitative arguments: for direct emissions, the only surviving term in the first line of eq. (A.5) is $\left(\boldsymbol{v}_{\perp}-\boldsymbol{u}_{L}\right)^{2} \simeq \theta_{q}^{2}$. To compute the spectrum at the formation time, one needs to perform the time integrations in eq. (A.1) without the factor $S_{g g}$ there, which describes multiple scattering after formation. For $\tau_{f} \ll L$ the integral over the time difference $x^{+}-y^{+}$is cut off by the factor $1 / B^{2}$ and yields a factor $\tau_{f}$, while the subsequent integral over $y^{+}$is unrestricted and yields a factor $L$. Altogether, we have a factor $\theta_{f}^{2} \tau_{f} L$ multiplying the Gaussian in eq. (A.6) plus, of course, the overall factor $\alpha_{s} C_{F}$. This reproduces the parametric estimate in eq. (2.8). 


\section{A.2 The final gluon spectrum}

The previous arguments also show that, in order to compute the final spectrum, as it would be measured by a detector, one needs to also take into account the $S$-matrix $S_{g g}$ of $g g$ dipole. Working in the momentum representation, the final spectrum is obtained by convoluting the spectrum at the time of formation with the Fourier transform of $S_{g g}$. Within the 'harmonic approximation', this amounts to an additional Gaussian broadening:

$$
F_{u_{L}, u_{F}}\left(x^{+}, y^{+}, \boldsymbol{k}_{\perp}\right)=\int \frac{\mathrm{d}^{2} \boldsymbol{q}_{\perp}}{(2 \pi)^{2}} I_{u_{L}, u_{F}}\left(x^{+}, y^{+}, \boldsymbol{q}_{\perp}\right) \frac{4 \pi}{Q_{s}^{2}} \mathrm{e}^{-\left(\boldsymbol{k}_{\perp}-\boldsymbol{q}_{\perp}\right)^{2} / Q_{s}^{2}}
$$

where $Q_{s}^{2} \equiv \hat{q}\left(L^{+}-x^{+}\right)$depends upon the final formation time $x^{+}$. Since the typical gluons are produced within the bulk of the medium $\left(x^{+} \ll L^{+}\right)$, one can neglect the $x^{+_{-}}$ dependence of $Q_{s}^{2}$ to parametric accuracy. After also using $\tau_{f} \ll L$, we find (with $Q_{s}^{2}=\hat{q} L^{+}$ from now on)

$$
\begin{aligned}
& F_{u_{L}, u_{F}}\left(x^{+}, y^{+}, \boldsymbol{k}_{\perp}\right) \approx \\
& \approx \frac{2 k^{+} \Omega}{B Q_{s}^{2}}\left[\frac{2}{B} \frac{\Omega}{i k^{+}}+\frac{y^{+} \Omega\left(\boldsymbol{u}_{L}-\boldsymbol{u}_{F}\right)^{2}}{\sinh \Omega\left(x^{+}-y^{+}\right)}\left(1+y^{+} \Omega\left(1+\frac{2}{B}\right)\right)-\right. \\
& \left.\quad 2 i \frac{\Omega}{Q_{s}^{2}}\left(\boldsymbol{k}_{\perp}-k^{+} \boldsymbol{u}_{L}\right) \cdot\left(\boldsymbol{u}_{L}-\boldsymbol{u}_{F}\right)\left(1+y^{+} \Omega\left(1+\frac{4}{B}\right)\right)\right] \times \\
& \quad \exp \left\{i \frac{k^{+}}{2}\left(\boldsymbol{u}_{L}-\boldsymbol{u}_{F}\right)^{2}\left(1+\Omega y^{+}\right) y^{+}-\frac{1}{Q_{s}^{2}}\left(\boldsymbol{k}_{\perp}-k^{+} \boldsymbol{u}_{L}-\frac{k^{+} \Omega y^{+}\left(\boldsymbol{u}_{L}-\boldsymbol{u}_{F}\right)}{\sinh \Omega\left(x^{+}-y^{+}\right)}\right)^{2}\right\},
\end{aligned}
$$

where we have further approximated $C \approx 1$ since we anticipate that $x^{+}-y^{+} \simeq \tau_{f}$. To understand eq. (A.9) to parametric accuracy, one can also set $B \approx 1$ and $\sinh \Omega\left(x^{+}-\right.$ $\left.y^{+}\right) / \Omega \approx \tau_{f}$.

As before, the first line of eq. (A.9) specifies the angular dependence of the final spectrum. Unlike in eq. (A.5), there is not a term proportional to the square of the final angle formed by the gluon and the quark. This is so since the final gluon spectrum receives contributions from the entire gluon spectrum at formation. The final distribution is characterized by $\theta_{f}$, the typical angle at formation, which can be identified in the first term of this line: $|\Omega| / k^{+}=1 /\left(\tau_{f} k^{+}\right) \simeq \theta_{f}^{2}$. For the interference term there is, however, a residual dependence upon the final direction of the gluon: this enters via the middle line of eq. (A.9), which is due to the fact that there is some correlation between the final direction of the gluon and its direction at the time of emission (as encoded in the off-set $k^{+} \boldsymbol{u}_{L}$ in the final gluon momentum). This term is essentially the same as that in eq. (5.22) from the main text and, as shown there, it is generally subleading.

The last line in eq. (A.9) encode both the time and momentum dependence. The first term in the exponent was already present in eq. (A.5) (the middle term in the exponent there) and, as already explained, it encodes the condition of quantum coherence - that is, the two time scales $\tau_{\lambda}$ and $\tau_{\text {int }}$. The second term in the exponent shows the transverse momentum spectrum, which is a Gaussian of width $Q_{s}^{2}$ around the direction $\boldsymbol{u}_{L}$ of the quark which participates in the formation process (i.e., the quark from the $q g$ dipole). The $\boldsymbol{q}_{\perp}$-dependence of the exponent in eq. (A.9) leads to a shift in the center of the transverse 
momentum gaussian; in fact, by using $\sinh \Omega\left(x^{+}-y^{+}\right) / \Omega \approx \tau_{f}$ to parametric accuracy, one sees that this additional shift is the same as the second term in the r.h.s. of eq. (5.15). As will be later verified, this additional shift is negligible in all the interesting cases.

Note finally that, as in the case of the spectrum at formation, eq. (A.9) must be supplemented with the $q q$ dipole eq. (5.3), which introduces the coherence time.

\section{A.3 Direct emission: the BDMPS-Z spectrum}

In the case of direct emission by either the quark or the antiquark, the final spectrum is obtained by integrating the following expression

$$
F_{q}\left(x^{+}, y^{+}, \boldsymbol{k}_{\perp}\right) \simeq \frac{4 \Omega^{2}}{i Q_{s}^{2}} \frac{1}{B^{2}} \exp \left\{-\frac{\left(\boldsymbol{k}_{\perp}-k^{+} \boldsymbol{u}_{\perp}\right)^{2}}{Q_{s}^{2}}\right\}
$$

over the time variables $x^{+}$and $y^{+}$. For definiteness, we have shown here the direct emission by the quark but there is of course a similar contribution by the antiquark. As expected, at this level of approximation the spectrum is a Gaussian centered around the transverse momentum $k^{+} \boldsymbol{u}_{\perp}$ inherited from the parent parton. As already explained, the subsequent integrations over the time variables introduce a factor $\tau_{f} L$. By also using $\tau_{f} \sim 1 /|\Omega|$ and $\theta_{f}^{2} \sim|\Omega| / k^{+}$we recover the parametric dependencies shown in eq. (4.21) of the main text.

\section{A.4 The interference terms for relatively large dipoles: $\theta_{f} \lesssim \theta_{q \bar{q}} \lesssim \theta_{s}$}

We shall now provide estimates for the interference contributions to the gluon spectrum, as obtained by integrating the expression in eq. (A.9) with $\boldsymbol{u}_{L} \neq \boldsymbol{u}_{F}$ over the time variables $x^{+}$and $y^{+}$. We first consider dipole angles within the range $\theta_{f} \lesssim \theta_{q \bar{q}} \lesssim \theta_{s}$. As before, the integration over $x^{+}-y^{+}$is dominated by $\tau_{f}$. But unlike the previous case of direct emissions, the $y^{+}$-integration is now more complicated since there are several competing time scales. As we have extensively discussed in section 2.2, within the present range for dipole angles, the relevant time scales are strictly ordered, $\tau_{\text {int }} \lesssim \tau_{\lambda} \lesssim \tau_{\text {coh }} \lesssim \tau_{f}$, and the integral over $y^{+}$is controlled by the smallest time scale, $\tau_{\text {int }}$. In addition, since $\tau_{\text {int }} / \tau_{f} \ll 1$, all terms proportional to $y^{+} \Omega$ in eq. (A.9) can be neglected. Then the only dependence upon $y^{+}$which survives in the exponent is that encoded in the first term there, $\simeq i k^{+} \theta_{q \bar{q}}^{2} y^{+}$, which after integration yields a factor $\tau_{\text {int }}$, as expected. The same approximations allow us to simplify the angular dependence of the final spectrum (the first line of eq. (A.9)) which contains terms proportional to $\theta_{f}^{2}, \theta_{q \bar{q}}^{2}$ and $\theta_{L} \theta_{q \bar{q}}$, where $\theta_{L}=\theta_{q}$ or $\theta_{\bar{q}}$ is the gluon angle with respect to the quark which enters the $q g$ dipole. By also using $|\Omega| / k^{+} \sim \theta_{f}^{2}$, $|\Omega| k^{+} \sim k_{f}^{2}$ and the following estimates,

$$
\tau_{\text {int }}|\Omega| \theta_{q \bar{q}}^{2} \sim \theta_{f}^{2}, \quad \frac{k_{f}^{2}}{Q_{s}^{2}} \theta_{L} \theta_{q \bar{q}} \sim \theta_{f}^{2} \frac{\theta_{q \bar{q}}^{2}}{\theta_{s}^{2}} \frac{\theta_{L}}{\theta_{q \bar{q}}}
$$

one eventually finds that the total interference term is parametrically given by

$$
\mathcal{I}^{(\mathrm{in})}\left(\omega, k_{\perp}\right) \propto-\alpha_{s} C_{F} \theta_{f}^{2}\left(1-c_{1} \frac{\theta_{q \bar{q}}^{2}}{\theta_{s}^{2}} \frac{\theta_{L}}{\theta_{q \bar{q}}}\right) \tau_{\mathrm{int}} \frac{\omega}{Q_{s}^{2}} \exp \left\{-\frac{\left(k_{\perp}-k^{+} \boldsymbol{u}_{L}\right)^{2}}{Q_{s}^{2}}\right\}
$$


where $c_{1}$ is a number of order one. We see that, unless $\theta_{L}$ is arbitrary large, $\theta_{L} \gg \theta_{q \bar{q}}$, the interference term, eq. (A.12), is suppressed with respect to the direct term, eq. (4.21), by $\tau_{\text {int }} / L^{+} \ll \tau_{f} L^{+} \ll 1$. Note that the term proportional to $c_{1}$ within the parentheses in eq. (A.12) is the same as that appearing in eq. (5.22) of the main text.

\section{A.5 The interference terms for relatively small dipoles: $\theta_{c} \ll \theta_{q \bar{q}} \ll \theta_{f}$}

Consider similarly the other relevant range for the dipole angles, at $\theta_{c} \ll \theta_{q \bar{q}} \ll \theta_{f}$. Then, as discussed in section 2.2 , the ordering of time scales gets now reverted, $\tau_{f} \ll \tau_{\text {coh }} \ll \tau_{\lambda} \ll \tau_{\text {int }}$ and the $y^{+}$integration is restricted by the smallest of the coherence time scales, that is $\tau_{\text {coh }}$. In this case, one we can safely neglect the $y^{+}$dependence of the exponential in eq. (A.9). (The integral over $y^{+}$is rather controlled by the $q \bar{q}$ dipole and yields a factor $\tau_{\text {coh. }}$.) In addition, since

$$
\frac{\left(k^{+}\right)^{2} \theta_{q \bar{q}}^{2}}{k_{f}^{2}} \frac{\tau_{\text {coh }}^{2}}{\tau_{f}^{2}} \sim\left(\frac{\theta_{q \bar{q}}}{\theta_{f}}\right)^{2 / 3} \ll 1
$$

we can neglect the shift in the transverse momentum Gaussian in eq. (A.9) for any final momentum $k_{\perp} \gtrsim k_{f}$. The overall magnitude of the interference term is controlled by the prefactor encoding the angular dependence (the first line in eq. (A.9)). In the present case $\tau_{\text {coh }}|\Omega| \gg 1$ and we need to determine the relative value of the three different contributions to the spectrum. Simple manipulations show that

$$
\left(\tau_{\operatorname{coh}} \theta_{q \bar{q}}|\Omega|\right)^{2} \sim \theta_{f}^{2}\left(\frac{\theta_{q \bar{q}}}{\theta_{f}}\right)^{2 / 3} \ll \theta_{f}^{2}, \quad \frac{k_{f}^{2}}{Q_{s}^{2}} \frac{\tau_{\mathrm{coh}}}{\tau_{f}} \theta_{L} \theta_{q \bar{q}} \sim \theta_{f}^{2} \frac{\theta_{L} \theta_{f}}{\theta_{s}^{2}}\left(\frac{\theta_{q \bar{q}}}{\theta_{f}}\right)^{1 / 3} .
$$

Using this expression and taking into account that the $x^{+}$and $y^{+}$integration lead to an overall factor of $\tau_{f} \tau_{\text {coh }}$ we can estimate the parametric dependence of the interference spectrum as

$$
\mathcal{I}^{(\mathrm{in})}\left(\omega, k_{\perp}\right) \propto-\alpha_{s} C_{F} \theta_{f}^{2}\left(1-c_{2} \frac{\theta_{L} \theta_{f}}{\theta_{s}^{2}}\left(\frac{\theta_{q \bar{q}}}{\theta_{f}}\right)^{1 / 3}\right) \tau_{\operatorname{coh}} \frac{\omega}{Q_{s}^{2}} \exp \left\{-\frac{\left(k_{\perp}-k^{+} \boldsymbol{u}_{L}\right)^{2}}{Q_{s}^{2}}\right\}
$$

where $c_{2}$ is a number of order 1 . It is then clear that, unless one considers gluons emitted at very large angles $\theta_{L} \gg \theta_{s}$, the interference term is proportional to $\theta_{f}^{2}$ and it is magnitude is suppressed as compared to the direct term by $\tau_{\text {coh }} / L \ll 1$ (cf. eq. (2.18)).

Open Access. This article is distributed under the terms of the Creative Commons Attribution Noncommercial License which permits any noncommercial use, distribution, and reproduction in any medium, provided the original author(s) and source are credited.

\section{References}

[1] ATLAS collaboration, G. Aad et al., Observation of a centrality-dependent dijet asymmetry in lead-lead collisions at $\sqrt{s_{N N}}=2.76 \mathrm{TeV}$ with the ATLAS detector at the LHC, Phys. Rev. Lett. 105 (2010) 252303 [arXiv: 1011.6182] [SPIRES].

[2] CMS collaboration, S. Chatrchyan et al., Observation and studies of jet quenching in $\mathrm{PbPb}$ collisions at nucleon-nucleon center-of-mass energy $=2.76 \mathrm{TeV}$, arXiv:1102.1957 [SPIRES]. 
[3] STAR collaboration, M. Ploskon, Inclusive cross section and correlations of fully reconstructed jets in $\sqrt{s_{N N}}=200 \mathrm{GeV} \mathrm{Au}+\mathrm{Au}$ and $p+p$ collisions,

Nucl. Phys. A 830 (2009) 255C [arXiv:0908.1799] [SPIRES].

[4] BRAHMS collaboration, I. Arsene et al., Quark gluon plasma an color glass condensate at RHIC? The perspective from the BRAHMS experiment, Nucl. Phys. A 757 (2005) 1 [nucl-ex/0410020] [SPIRES].

[5] B.B. Back et al., The PHOBOS perspective on discoveries at RHIC, Nucl. Phys. A 757 (2005) 28 [nucl-ex/0410022] [SPIRES].

[6] STAR collaboration, J. Adams et al., Experimental and theoretical challenges in the search for the quark gluon plasma: the STAR collaboration's critical assessment of the evidence from RHIC collisions, Nucl. Phys. A 757 (2005) 102 [nucl-ex/0501009] [SPIRES].

[7] PHENIX collaboration, K. Adcox et al., Formation of dense partonic matter in relativistic nucleus nucleus collisions at RHIC: experimental evaluation by the PHENIX collaboration, Nucl. Phys. A 757 (2005) 184 [nucl-ex/0410003] [SPIRES].

[8] ALICE collaboration, K. Aamodt et al., Suppression of charged particle production at large transverse momentum in central Pb-Pb collisions at $\sqrt{s_{N N}}=2.76 \mathrm{TeV}$, Phys. Lett. B 696 (2011) 30 [arXiv:1012.1004] [SPIRES].

[9] R. Baier, Y.L. Dokshitzer, A.H. Mueller, S. Peigne and D. Schiff, Radiative energy loss of high energy quarks and gluons in a finite-volume quark-gluon plasma, Nucl. Phys. B 483 (1997) 291 [hep-ph/9607355] [SPIRES].

[10] R. Baier, Y.L. Dokshitzer, A.H. Mueller, S. Peigne and D. Schiff, Radiative energy loss and $p_{T}$-broadening of high energy partons in nuclei, Nucl. Phys. B 484 (1997) 265 [hep-ph/9608322] [SPIRES].

[11] R. Baier, Y.L. Dokshitzer, A.H. Mueller and D. Schiff, Radiative energy loss of high energy partons traversing an expanding QCD plasma, Phys. Rev. C 58 (1998) 1706 [hep-ph/9803473] [SPIRES].

[12] B.G. Zakharov, Fully quantum treatment of the Landau-Pomeranchuk-Migdal effect in QED and QCD, JETP Lett. 63 (1996) 952 [hep-ph/9607440] [SPIRES].

[13] M. Gyulassy, P. Levai and I. Vitev, Reaction operator approach to non-abelian energy loss, Nucl. Phys. B 594 (2001) 371 [nucl-th/0006010] [SPIRES].

[14] U.A. Wiedemann, Gluon radiation off hard quarks in a nuclear environment: opacity expansion, Nucl. Phys. B 588 (2000) 303 [hep-ph/0005129] [SPIRES].

[15] C.A. Salgado and U.A. Wiedemann, Medium modification of jet shapes and jet multiplicities, Phys. Rev. Lett. 93 (2004) 042301 [hep-ph/0310079] [SPIRES].

[16] E. Wang and X.-N. Wang, Jet tomography of dense and nuclear matter, Phys. Rev. Lett. 89 (2002) 162301 [hep-ph/0202105] [SPIRES].

[17] P.B. Arnold, G.D. Moore and L.G. Yaffe, Photon and gluon emission in relativistic plasmas, JHEP 06 (2002) 030 [hep-ph/0204343] [SPIRES].

[18] R. Baier, D. Schiff and B.G. Zakharov, Energy loss in perturbative QCD, Ann. Rev. Nucl. Part. Sci. 50 (2000) 37 [hep-ph/0002198] [SPIRES].

[19] J. Casalderrey-Solana and C.A. Salgado, Introductory lectures on jet quenching in heavy ion collisions, Acta Phys. Polon. B 38 (2007) 3731 [arXiv:0712.3443] [SPIRES].

[20] D. d'Enterria, Jet quenching, arXiv:0902.2011 [SPIRES]. 
[21] A. Majumder and M. Van Leeuwen, The theory and phenomenology of perturbative $Q C D$ based jet quenching, Prog. Part. Nucl. Phys. 66 (2011) 41 [arXiv:1002.2206] [SPIRES].

[22] J. Casalderrey-Solana, J.G. Milhano and U.A. Wiedemann, Jet quenching via jet collimation, J. Phys. G 38 (2011) 035006 [arXiv:1012.0745] [SPIRES].

[23] G.-Y. Qin and B. Müller, Explanation of di-jet asymmetry in $\mathrm{Pb}+\mathrm{Pb}$ collisions at the Large Hadron Collider, Phys. Rev. Lett. 106 (2011) 162302 [arXiv:1012.5280] [SPIRES].

[24] I.P. Lokhtin, A.V. Belyaev and A.M. Snigirev, Jet quenching pattern at LHC in PYQUEN model, Eur. Phys. J. C 71 (2011) 1650 [arXiv:1103.1853] [SPIRES].

[25] C. Young, B. Schenke, S. Jeon and C. Gale, Dijet asymmetry at the Large Hadron Collider, arXiv:1103.5769 [SPIRES].

[26] Y. Mehtar-Tani, C.A. Salgado and K. Tywoniuk, Antiangular ordering of gluon radiation in QCD media, Phys. Rev. Lett. 106 (2011) 122002 [arXiv: 1009.2965] [SPIRES].

[27] Y. Mehtar-Tani, C.A. Salgado and K. Tywoniuk, Jets in QCD media: from color coherence to decoherence, arXiv:1102.4317 [SPIRES].

[28] Y.L. Dokshitzer, V.A. Khoze, A.H. Mueller and S.I. Troian, Basics of perturbative QCD, Ed. Frontieres, Gif-sur-Yvette France (1991) [SPIRES].

[29] R.K. Ellis, W.J. Stirling and B.R. Webber, QCD and collider physics, Camb. Monogr. Part. Phys. Nucl. Phys. Cosmol. 8 (1996) 1 [SPIRES].

[30] E. Iancu and R. Venugopalan, The color glass condensate and high energy scattering in $Q C D$, in Quark Gluon Plasma 3, R.C. Hwa and X.N. Wang eds., World Scientific (2004) [hep-ph/0303204] [SPIRES].

[31] P.B. Arnold, Simple formula for high-energy gluon bremsstrahlung in a finite, expanding medium, Phys. Rev. D 79 (2009) 065025 [arXiv: 0808.2767] [SPIRES].

[32] Y. Mehtar-Tani and K. Tywoniuk, Jet coherence in QCD media: the antenna radiation spectrum, arXiv:1105.1346 [SPIRES].

[33] Y. Mehtar-Tani, Relating the description of gluon production in $p A$ collisions and parton energy loss in AA collisions, Phys. Rev. C 75 (2007) 034908 [hep-ph/0606236] [SPIRES].

[34] I.P. Lokhtin and A.M. Snigirev, A model of jet quenching in ultrarelativistic heavy ion collisions and high-p T $_{T}$ hadron spectra at RHIC, Eur. Phys. J. C 45 (2006) 211 [hep-ph/0506189] [SPIRES].

[35] K. Zapp, J. Stachel and U.A. Wiedemann, A local Monte Carlo implementation of the non-abelian Landau-Pomerantschuk-Migdal effect, Phys. Rev. Lett. 103 (2009) 152302 [arXiv: 0812.3888] [SPIRES].

[36] N. Armesto, L. Cunqueiro and C.A. Salgado, Q-PYTHIA - a Monte Carlo implementation for jet quenching, arXiv:0906.0754 [SPIRES].

[37] N. Armesto, G. Corcella, L. Cunqueiro and C.A. Salgado, Angular-ordered parton showers with medium-modified splitting functions, JHEP 11 (2009) 122 [arXiv:0909.5118] [SPIRES].

[38] B. Schenke, C. Gale and S. Jeon, MARTINI: an event generator for relativistic heavy-ion collisions, Phys. Rev. C 80 (2009) 054913 [arXiv: 0909. 2037] [SPIRES].

[39] T. Renk, YaJEM - a Monte Carlo code for in-medium shower evolution, arXiv:1009.3740 [SPIRES].

[40] A. Leonidov and V. Nechitailo, Decoherence and energy loss in QCD cascades in nuclear collisions, Eur. Phys. J. C 71 (2011) 1537 [arXiv: 1006.0366] [SPIRES]. 